\title{
Positive Solutions for a Third Order Nonlinear Neutral Delay Difference Equation
}

\author{
Zeqing Liu, ${ }^{1}$ Xiaochen Wang, ${ }^{1}$ Shin Min Kang, ${ }^{2}$ and Young Chel Kwun ${ }^{3}$ \\ ${ }^{1}$ Department of Mathematics, Liaoning Normal University, Dalian, Liaoning 116029, China \\ ${ }^{2}$ Department of Mathematics and RINS, Gyeongsang National University, Jinju 660-701, Republic of Korea \\ ${ }^{3}$ Department of Mathematics, Dong-A University, Busan 614-714, Republic of Korea
}

Correspondence should be addressed to Young Chel Kwun; yckwun@dau.ac.kr

Received 8 August 2014; Accepted 20 December 2014

Academic Editor: Chuanzhi Bai

Copyright (C) 2015 Zeqing Liu et al. This is an open access article distributed under the Creative Commons Attribution License, which permits unrestricted use, distribution, and reproduction in any medium, provided the original work is properly cited.

The existence, multiplicity, and properties of positive solutions for a third order nonlinear neutral delay difference equation are discussed. Six examples are given to illustrate the results presented in this paper.

\section{Introduction and Preliminaries}

Recently, some researchers used the Reccati transformation techniques, fixed point theorems, and iterative algorithms to study the oscillation, nonoscillation, asymptotic properties, and solvability for linear and nonlinear third order difference equations and systems; see, for example, [1-6] and the references therein. In particular, Saker [4], Andruch-Sobilo and Migda [1], and Grace and Hamedani [2] discussed the oscillation for the following third order difference equations:

$$
\begin{aligned}
& \Delta^{3} x(n)+p(n) x(n+1)=0, n \geq n_{0}, \\
& \Delta^{3}(x(n)-p(n) x(\sigma(n))) \pm q(n) x(\tau(n))=0, n \geq n_{0}, \\
& \Delta^{3}(x(n)-x(n-\tau)) \pm q(n)|x(n-\sigma)|^{3} \operatorname{sgn} x(n-\sigma)=0, \\
& n \geq 0 .
\end{aligned}
$$

Making use of the Schauder fixed point theorem, Banach fixed point theorem, and Mann iterative schemes, Yan and Liu [5] and Liu et al. [3], respectively, proved the existence of a bounded nonoscillatory solution for the third order difference equation:

$$
\Delta^{3} x(n)+f(n, x(n), x(n-\tau))=0, \quad n \geq n_{0}
$$

and the existence of positive solutions and convergence of the Mann iterative schemes for the following third order nonlinear neutral delay difference equation:

$$
\begin{aligned}
\Delta^{3}(x(n) & +b(n) x(n-\tau)) \\
& +\Delta h\left(n, x\left(h_{1}(n)\right), x\left(h_{2}(n)\right), \ldots, x\left(h_{k}(n)\right)\right) \\
& +f\left(n, x\left(f_{1}(n)\right), x\left(f_{2}(n)\right), \ldots, x\left(f_{k}(n)\right)\right)=c(n),
\end{aligned}
$$$$
n \geq n_{0} \text {. }
$$

However, the following third order nonlinear neutral delay difference equation:

$$
\Delta^{3}(x(n)+b(n) x(n-\tau)+c(n))
$$

$$
\begin{aligned}
& +\Delta^{2} h\left(n, x\left(h_{1}(n)\right), \ldots, x\left(h_{k}(n)\right)\right) \\
& +\Delta g\left(n, x\left(g_{1}(n)\right), \ldots, x\left(g_{k}(n)\right)\right) \\
& +f\left(n, x\left(f_{1}(n)\right), \ldots, x\left(f_{k}(n)\right)\right)=d(n),
\end{aligned}
$$

$$
n \geq n_{0} \text {, }
$$


where $\tau, k, n_{0} \in \mathbb{N},\{c(n)\}_{n \in \mathbb{N}_{n_{0}}},\{b(n)\}_{n \in \mathbb{N}_{n_{0}}}$, and $\{d(n)\}_{n \in \mathbb{N}_{n_{0}}}$ are real sequences, $f, g, h \in C\left(\mathbb{N} \times \mathbb{R}^{k}, \mathbb{R}\right)$ and $f_{l}, g_{l}, h_{l}: \mathbb{N}_{n_{0}} \rightarrow \mathbb{N}$ with

$$
\begin{array}{r}
\lim _{n \rightarrow \infty} f_{l}(n)=\lim _{n \rightarrow \infty} g_{l}(n)=\lim _{n \rightarrow \infty} h_{l}(n)=+\infty, \\
l \in\{1,2, \ldots, k\}
\end{array}
$$

has not been studied. The purpose of this paper is to study solvability of (4). By utilizing the Krasnoselskii fixed point theorem, Schauder fixed point theorem and some new techniques, we establish the existence, multiplicity, and properties of positive solutions of (4). Six examples are constructed to illuminate our results.

Throughout this paper, we assume that $\Delta$ is the forward difference operator defined by $\Delta x(n)=x(n+1)-x(n), \mathbb{R}=$ $(-\infty,+\infty), \mathbb{R}^{+}=[0,+\infty)$, and $\mathbb{N}_{0}$ and $\mathbb{N}$ denote the sets of nonnegative integers and positive integers, respectively,

$$
\begin{gathered}
\mathbb{N}_{t}=\left\{n: n \in \mathbb{N}_{0} \text { with } n \geq t\right\}, \quad t \in \mathbb{N}_{0}, \\
\alpha=\inf \left\{f_{l}(n), g_{l}(n), h_{l}(n): 1 \leq l \leq k, n \in \mathbb{N}_{n_{0}}\right\}, \\
\beta=\min \left\{\left|n_{0}-\tau\right|, \alpha\right\} \in \mathbb{N} .
\end{gathered}
$$

Let $l_{\beta}^{\infty}$ denote the Banach space of all real sequences $x=$ $\{x(n)\}_{n \in \mathbb{N}_{\beta}}$ with norm

$$
\|x\|=\sup _{n \in \mathbb{N}_{\beta}}\left|\frac{x(n)}{n}\right|<+\infty \quad \text { for } x=\{x(n)\}_{n \in \mathbb{N}_{\beta}} \in l_{\beta}^{\infty} .
$$

Let $A, B, A_{*}, B^{*}$ and $c^{*}$ be positive constants, $T \in \mathbb{N}$, $\{c(n)\}_{n \in \mathbb{N}_{\beta}},\{A(n)\}_{n \in \mathbb{N}_{\beta}}$, and $\{B(n)\}_{n \in \mathbb{N}_{\beta}}$ be real sequences with

$$
\begin{gathered}
B(n)=B+\frac{|c(n)|}{n}>A(n)=A-\frac{|c(n)|}{n}, \quad n \in \mathbb{N}_{\beta}, \\
c(n)=c\left(n_{0}\right), \quad \beta \leq n \leq n_{0}-1, \\
c^{*} \geq \sup _{n \in \mathbb{N}_{\beta}} \frac{|c(n)|}{n}, \\
A_{*}=A-c^{*}, \quad B^{*}=B+c^{*} .
\end{gathered}
$$

Put

$$
\begin{gathered}
\Omega\left(A_{*}, B^{*}, T\right) \\
=\left\{x=\{x(n)\}_{n \in \mathbb{N}_{\beta}} \in l_{\beta}^{\infty}: A(T) \leq \frac{x(n)}{n} \leq B(T),\right. \\
\left.\quad \beta \leq n<T ; A(n) \leq \frac{x(n)}{n} \leq B(n), n \geq T\right\} .
\end{gathered}
$$

It is easy to see that $\Omega\left(A_{*}, B^{*}, T\right)$ is a bounded closed and convex subset of $l_{\beta}^{\infty}$.

By a solution of (4), we mean a sequence $\{x(n)\}_{n \in \mathbb{N}_{\beta}} \in l_{\beta}^{\infty}$ with a positive integer $T \geq n_{0}+\tau+\alpha$ such that (4) holds for all $n \geq T$.

The following lemmas play important roles in this paper.
Lemma 1 (see [6]). A bounded and uniformly Cauchy subset $C \subseteq l_{\beta}^{\infty}$ is relatively compact.

Lemma 2 (Krasnoselskii fixed point theorem). Let $X$ be a Banach space, $D$ a bounded closed convex subset of $X$, and $S, G: D \rightarrow X$ mappings such that $S x+G y \in D$ for every pair $x, y \in D$. If $S$ is a contraction and $G$ is completely continuous, then the equation

$$
S x+G x=x
$$

has a solution in $D$.

Lemma 3 (Schauder fixed point theorem). Let $D$ be a nonempty closed convex subset of a Banach space $X$ and $T$ : $D \rightarrow D$ a continuous mapping such that $T(D)$ is a relatively compact subset of $X$. Then $T$ has at least one fixed point in $D$.

Lemma 4. Let $\tau, n \in \mathbb{N}$ and $\{q(k)\}_{k \in \mathbb{N}} \subset \mathbb{R}^{+}$. Then

(i) $\sum_{i=1}^{\infty} \sum_{t=n+i \tau}^{\infty} q(t) \leq \sum_{t=n+\tau}^{\infty}(t / \tau) q(t)$;

(ii) $\sum_{i=1}^{\infty} \sum_{s=n+i \tau}^{\infty} \sum_{t=s}^{\infty} q(t) \leq \sum_{t=n+\tau}^{\infty}\left(t^{2} / \tau\right) q(t)$;

(iii) $\sum_{p=1}^{\infty} \sum_{i=n+p \tau}^{\infty} \sum_{s=i}^{\infty} \sum_{t=s}^{\infty} q(t) \leq \sum_{t=n+\tau}^{\infty}\left(t^{3} / \tau\right) q(t)$.

Proof. (i) Let $[t]$ denote the largest integer number not exceeding $t \in \mathbb{R}^{+}$. It is clear that

$$
\begin{aligned}
\sum_{i=1}^{\infty} \sum_{t=n+i \tau}^{\infty} q(t) & =\sum_{t=n+\tau}^{\infty}\left(1+\left[\frac{t-n-\tau}{\tau}\right]\right) q(t) \\
& \leq \sum_{t=n+\tau}^{\infty} \frac{t}{\tau} q(t) .
\end{aligned}
$$

(ii) It follows from (i) that

$$
\begin{aligned}
\sum_{i=1}^{\infty} \sum_{s=n+i \tau}^{\infty} \sum_{t=s}^{\infty} q(t) & =\sum_{i=1}^{\infty} \sum_{t=n+i \tau}^{\infty}(1+t-n-i \tau) q(t) \\
& \leq \sum_{i=1}^{\infty} \sum_{t=n+i \tau}^{\infty} t q(t) \leq \sum_{t=n+\tau}^{\infty} \frac{t^{2}}{\tau} q(t) .
\end{aligned}
$$

(iii) It follows from (ii) that

$$
\begin{aligned}
\sum_{p=1}^{\infty} \sum_{i=n+p \tau}^{\infty} \sum_{s=i}^{\infty} \sum_{t=s}^{\infty} q(t) & =\sum_{p=1}^{\infty} \sum_{i=n+p \tau}^{\infty} \sum_{t=i}^{\infty}(t-i+1) q(t) \\
& \leq \sum_{p=1}^{\infty} \sum_{i=n+p \tau}^{\infty} \sum_{t=i}^{\infty} t q(t) \leq \sum_{t=n+\tau}^{\infty} \frac{t^{3}}{\tau} q(t) .
\end{aligned}
$$

This completes the proof.

\section{Existence of Positive Solutions}

Now we discuss the existence, multiplicity, and properties of positive solutions of (4) under various conditions on the sequence $\{b(n)\}_{n \in \mathbb{N}_{\beta}} \subseteq \mathbb{R}$. 
Theorem 5. Assume that there exist $a$ constant $b^{*}$ and three nonnegative sequences $\left\{F_{n}\right\}_{n \in \mathbb{N}_{n_{0}}},\left\{G_{n}\right\}_{n \in \mathbb{N}_{n_{0}}}$, and $\left\{H_{n}\right\}_{n \in \mathbb{N}_{n_{0}}}$ satisfying

$$
\begin{array}{r}
A+b^{*} B^{*}<B, \quad 0 \leq b(n) \leq b^{*}<1, n \in \mathbb{N}_{n_{0}}, \\
\left|f\left(n, u_{1}, u_{2}, \ldots, u_{k}\right)\right| \leq F_{n}, \\
\left|g\left(n, u_{1}, u_{2}, \ldots, u_{k}\right)\right| \leq G_{n} \\
\left|h\left(n, u_{1}, u_{2}, \ldots, u_{k}\right)\right| \leq H_{n} \\
\left(n, u_{l}\right) \in \mathbb{N}_{n_{0}} \times\left(\mathbb{R}^{+} \backslash\{0\}\right), \quad 1 \leq l \leq k \\
\lim _{n \rightarrow \infty} \frac{1}{n} \sum_{i=n}^{\infty} \max \left\{H_{i}, \sum_{s=i}^{\infty} G_{s}\right\}=0 \\
\lim _{n \rightarrow \infty} \frac{1}{n} \sum_{i=n}^{\infty} \sum_{s=i}^{\infty} \sum_{t=s}^{\infty} \max \left\{F_{t},|d(t)|\right\}=0 .
\end{array}
$$

Then

(i) equation (4) possesses a positive solution $x=$ $\{x(n)\}_{n \in \mathbb{N}_{\beta}} \in l_{\beta}^{\infty}$ with

$$
\lim _{n \rightarrow \infty} \frac{x(n)+b(n) x(n-\tau)+c(n)}{n} \in\left(A+b^{*} B^{*}, B\right),
$$

$$
A_{*} \leq \liminf _{n \rightarrow \infty} \frac{x(n)}{n} \leq \limsup _{n \rightarrow \infty} \frac{x(n)}{n} \leq B^{*}
$$

(ii) equation (4) possesses uncountably many positive solutions in $l_{\beta}^{\infty}$.

Proof. (i) Let $L \in\left(A+b^{*} B^{*}, B\right)$. It follows from (16) and (17) that there exists $T \geq n_{0}+\tau+\alpha$ satisfying

$$
\begin{gathered}
\frac{1}{T} \sum_{i=T}^{\infty}\left\{H_{i}+\sum_{s=i}^{\infty} G_{s}+\sum_{s=i}^{\infty} \sum_{t=s}^{\infty}\left[F_{t}+|d(t)|\right]\right\} \\
<\min \left\{L-A-b^{*} B^{*}, B-L\right\} .
\end{gathered}
$$

Define two mappings $S_{L}$ and $G_{L}: \Omega\left(A_{*}, B^{*}, T\right) \rightarrow l_{\beta}^{\infty}$ by

$$
\left(S_{L} x\right)(n)= \begin{cases}n L-b(n) x(n-\tau)-c(n), & n \geq T, \\ \frac{n}{T}\left(S_{L} x\right)(T), & \beta \leq n<T,\end{cases}
$$

$$
\left(G_{L} x\right)(n)=\left\{\begin{array}{c}
\sum_{i=n}^{\infty} h\left(i, x\left(h_{1}(i)\right), \ldots, x\left(h_{k}(i)\right)\right) \\
-\sum_{i=n}^{\infty} \sum_{s=i}^{\infty} g\left(s, x\left(g_{1}(s)\right), \ldots, x\left(g_{k}(s)\right)\right) \\
+\sum_{i=n}^{\infty} \sum_{s=i}^{\infty} \sum_{t=s}^{\infty}\left[f \left(t, x\left(f_{1}(t)\right), \ldots,\right.\right. \\
\left.\left.x\left(f_{k}(t)\right)\right)-d(t)\right], \\
n \geq T, \\
\frac{n}{T}\left(G_{L} x\right)(T), \quad \beta<T,
\end{array}\right.
$$

for any $x=\{x(n)\}_{n \in \mathbb{N}_{\beta}} \in \Omega\left(A_{*}, B^{*}, T\right)$.

Now we show that

$$
S_{L} x+G_{L} y \in \Omega\left(A_{*}, B^{*}, T\right), \quad x, y \in \Omega\left(A_{*}, B^{*}, T\right) ;
$$

$$
\left\|S_{L} x-S_{L} y\right\| \leq b^{*}\|x-y\|, \quad x, y \in \Omega\left(A_{*}, B^{*}, T\right),
$$

$$
\left\|G_{L} y\right\| \leq B, \quad y \in \Omega\left(A_{*}, B^{*}, T\right) .
$$

Using (14), (15), and (20)-(22), we get that for any $x=$ $\{x(n)\}_{n \in \mathbb{N}_{\beta}}, y=\{y(n)\}_{n \in \mathbb{N}_{\beta}} \in \Omega\left(A_{*}, B^{*}, T\right)$

$$
\begin{aligned}
& \frac{\left(S_{L} x\right)(n)+\left(G_{L} y\right)(n)}{n} \\
& =L-\frac{b(n)}{n} x(n-\tau)-\frac{c(n)}{n} \\
& \quad+\frac{1}{n} \sum_{i=n}^{\infty} h\left(i, y\left(h_{1}(i)\right), \ldots, y\left(h_{k}(i)\right)\right) \\
& \quad-\frac{1}{n} \sum_{i=n}^{\infty} \sum_{s=i}^{\infty} g\left(s, y\left(g_{1}(s)\right), \ldots, y\left(g_{k}(s)\right)\right) \\
& \quad+\frac{1}{n} \sum_{i=n}^{\infty} \sum_{s=i}^{\infty} \sum_{t=s}^{\infty}\left[f\left(t, x\left(f_{1}(t)\right), \ldots, x\left(f_{k}(t)\right)\right)-d(t)\right] \\
& \leq L+\frac{|c(n)|}{n}
\end{aligned}
$$

$$
\begin{aligned}
& +\frac{1}{n} \sum_{i=n}^{\infty}\left|h\left(i, y\left(h_{1}(i)\right), \ldots, y\left(h_{k}(i)\right)\right)\right| \\
& +\frac{1}{n} \sum_{i=n}^{\infty} \sum_{s=i}^{\infty}\left|g\left(s, y\left(g_{1}(s)\right), \ldots, y\left(g_{k}(s)\right)\right)\right|
\end{aligned}
$$




$$
\begin{aligned}
& +\frac{1}{n} \sum_{i=n}^{\infty} \sum_{s=i}^{\infty} \sum_{t=s}^{\infty}\left[\left|f\left(t, y\left(f_{1}(t)\right), \ldots, y\left(f_{k}(t)\right)\right)\right|+|d(t)|\right] \\
& \leq L+\frac{|c(n)|}{n}+\frac{1}{T} \sum_{i=T}^{\infty} H_{i}+\frac{1}{T} \sum_{i=T}^{\infty} \sum_{s=i}^{\infty} G_{s} \\
& +\frac{1}{T} \sum_{i=T}^{\infty} \sum_{s=i}^{\infty} \sum_{t=s}^{\infty}\left[F_{t}+|d(t)|\right] \\
& <L+\frac{|c(n)|}{n}+\min \left\{L-A-b^{*} B^{*}, B-L\right\} \\
& \leq B+\frac{|c(n)|}{n}=B(n), \quad n \geq T, \\
& \frac{\left(S_{L} x\right)(n)+\left(G_{L} y\right)(n)}{n} \\
& =\frac{n}{T} \cdot \frac{\left(S_{L} x\right)(T)+\left(G_{L} y\right)(T)}{n} \leq B(T), \quad \beta \leq n<T, \\
& \frac{\left(S_{L} x\right)(n)+\left(G_{L} y\right)(n)}{n} \\
& =L-\frac{b(n)}{n} x(n-\tau)-\frac{c(n)}{n} \\
& +\frac{1}{n} \sum_{i=n}^{\infty} h\left(i, y\left(h_{1}(i)\right), \ldots, y\left(h_{k}(i)\right)\right) \\
& -\frac{1}{n} \sum_{i=n}^{\infty} \sum_{s=i}^{\infty} g\left(s, y\left(g_{1}(s)\right), \ldots, y\left(g_{k}(s)\right)\right) \\
& +\frac{1}{n} \sum_{i=n}^{\infty} \sum_{s=i}^{\infty} \sum_{t=s}^{\infty}\left[f\left(t, y\left(f_{1}(t)\right), \ldots, y\left(f_{k}(t)\right)\right)-d(t)\right] \\
& \geq L-\frac{n-\tau}{n} b(n) \frac{x(n-\tau)}{n-\tau}-\frac{|c(n)|}{n} \\
& -\frac{1}{n} \sum_{i=n}^{\infty}\left|h\left(i, y\left(h_{1}(i)\right), \ldots, y\left(h_{k}(i)\right)\right)\right| \\
& -\frac{1}{n} \sum_{i=n}^{\infty} \sum_{s=i}^{\infty}\left|g\left(s, y\left(g_{1}(s)\right), \ldots, y\left(g_{k}(s)\right)\right)\right| \\
& -\frac{1}{n} \sum_{i=n}^{\infty} \sum_{s=i}^{\infty} \sum_{t=s}^{\infty}\left[\left|f\left(t, y\left(f_{1}(t)\right), \ldots, y\left(f_{k}(t)\right)\right)\right|+|d(t)|\right] \\
& \geq L-b^{*} B^{*}-\frac{|c(n)|}{n} \\
& -\frac{1}{T} \sum_{i=T}^{\infty} H_{i}-\frac{1}{T} \sum_{i=T}^{\infty} \sum_{s=i}^{\infty} G_{s}
\end{aligned}
$$

$$
\begin{gathered}
-\frac{1}{T} \sum_{i=T}^{\infty} \sum_{s=i}^{\infty} \sum_{t=s}^{\infty}\left[F_{t}+|d(t)|\right] \\
>L-b^{*} B^{*}-\frac{|c(n)|}{n} \\
\quad-\min \left\{L-A-b^{*} B^{*}, B-L\right\} \\
\geq A-\frac{|c(n)|}{n}=A(n), \quad n \geq T,
\end{gathered}
$$$$
\frac{\left(S_{L} x\right)(n)+\left(G_{L} y\right)(n)}{n}
$$$$
=\frac{n}{T} \cdot \frac{\left(S_{L} x\right)(T)+\left(G_{L} y\right)(T)}{n} \geq A(T), \quad \beta \leq n<T,
$$$$
\left|\frac{\left(S_{L} x\right)(n)-\left(S_{L} y\right)(n)}{n}\right|=b(n) \frac{n-\tau}{n}\left|\frac{x(n-\tau)-y(n-\tau)}{n-\tau}\right|
$$$$
\leq b^{*}\|x-y\|, \quad n \geq T,
$$$$
\left|\frac{\left(S_{L} x\right)(n)-\left(S_{L} y\right)(n)}{n}\right|=\frac{n}{T}\left|\frac{\left(S_{L} x\right)(T)-\left(S_{L} y\right)(T)}{n}\right|
$$$$
\leq b^{*}\|x-y\|, \quad \beta \leq n<T,
$$$$
\left|\frac{\left(G_{L} y\right)(n)}{n}\right|
$$$$
=\mid \frac{1}{n} \sum_{i=n}^{\infty} h\left(i, y\left(h_{1}(i)\right), \ldots, y\left(h_{k}(i)\right)\right)
$$$$
-\frac{1}{n} \sum_{i=n}^{\infty} \sum_{s=i}^{\infty} g\left(s, y\left(g_{1}(s)\right), \ldots, y\left(g_{k}(s)\right)\right)
$$$$
+\frac{1}{n} \sum_{i=n}^{\infty} \sum_{s=i}^{\infty} \sum_{t=s}^{\infty}\left[f\left(t, y\left(f_{1}(t)\right), \ldots, y\left(f_{k}(t)\right)\right)\right.
$$$$
-d(t)]
$$$$
\leq \frac{1}{n} \sum_{i=n}^{\infty}\left|h\left(i, y\left(h_{1}(i)\right), \ldots, y\left(h_{k}(i)\right)\right)\right|
$$$$
+\frac{1}{n} \sum_{i=n}^{\infty} \sum_{s=i}^{\infty}\left|g\left(s, y\left(g_{1}(s)\right), \ldots, y\left(g_{k}(s)\right)\right)\right|
$$$$
+\frac{1}{n} \sum_{i=n}^{\infty} \sum_{s=i}^{\infty} \sum_{t=s}^{\infty}\left[\left|f\left(t, y\left(f_{1}(t)\right), \ldots, y\left(f_{k}(t)\right)\right)\right|\right.
$$

$$
+|d(t)|]
$$




$$
\begin{aligned}
& \leq \frac{1}{T} \sum_{i=T}^{\infty} H_{i}+\frac{1}{T} \sum_{i=T}^{\infty} \sum_{s=i}^{\infty} G_{s} \\
& \quad+\frac{1}{T} \sum_{i=T}^{\infty} \sum_{s=i}^{\infty} \sum_{t=s}^{\infty}\left[F_{t}+|d(t)|\right] \\
& <\min \left\{L-A-b^{*} B^{*}, B-L\right\} \leq B, \quad n \geq T, \\
& \left|\frac{\left(G_{L} y\right)(n)}{n}\right|=\frac{n}{T}\left|\frac{\left(G_{L} y\right)(T)}{n}\right| \leq B, \quad \beta \leq n<T,
\end{aligned}
$$

which yield the fact that (23)-(25) hold.

Next we show that $G_{L}$ is completely continuous. Let $y^{w}=$ $\left\{y^{w}(n)\right\}_{n \in \mathbb{N}_{\beta}}$ and $y=\{y(n)\}_{n \in \mathbb{N}_{\beta}} \in \Omega\left(A_{*}, B^{*}, T\right)$ with

$$
\lim _{w \rightarrow \infty} y^{w}=y
$$

Using (16), (17), (27), and the continuity of $f, g$, and $h$, we know that for given $\varepsilon>0$, there exist $T_{1}, T_{2}, T_{3}$ and $T_{4} \in \mathbb{N}$ with $T_{4}>T_{3}>T_{2}>T_{1}>T$ satisfying

$$
\begin{aligned}
& \frac{1}{T} \max \left\{\sum_{i=T_{1}+1}^{\infty} H_{i}+\sum_{i=T_{1}+1}^{\infty} \sum_{s=i}^{\infty} G_{s}\right. \\
& +\sum_{i=T_{1}+1}^{\infty} \sum_{s=i}^{\infty} \sum_{t=s}^{\infty}\left[F_{t}+|d(t)|\right] \\
& \sum_{i=T}^{T_{1}} \sum_{s=T_{2}+1}^{\infty} G_{s}+\sum_{i=T}^{T_{1}} \sum_{s=T_{2}+1}^{\infty} \sum_{t=s}^{\infty} F_{t} \\
& \left.+\sum_{i=T}^{T_{1}} \sum_{s=i}^{T_{2}} \sum_{t=T_{3}+1}^{\infty} F_{t}\right\}<\frac{\varepsilon}{16} \\
& \frac{1}{T} \max \left\{\sum_{i=T}^{T_{1}} \mid h\left(i, y^{w}\left(h_{1}(i)\right), \ldots, y^{w}\left(h_{k}(i)\right)\right)\right. \\
& -h\left(i, y\left(h_{1}(i)\right), \ldots, y\left(h_{k}(i)\right)\right) \mid, \\
& \sum_{i=T}^{T_{1}} \sum_{s=i}^{T_{2}} \mid g\left(s, y^{w}\left(g_{1}(s)\right), \ldots, y^{w}\left(g_{k}(s)\right)\right) \\
& -g\left(s, y\left(g_{1}(s)\right), \ldots, y\left(g_{k}(s)\right)\right) \mid, \\
& \sum_{i=T}^{T_{1}} \sum_{s=i}^{T_{2}} \sum_{t=s}^{T_{3}} \mid f\left(t, y^{w}\left(f_{1}(t)\right), \ldots, y^{w}\left(f_{k}(t)\right)\right) \\
& \left.-f\left(t, y\left(f_{1}(t)\right), \ldots, y\left(f_{k}(t)\right)\right) \mid\right\} \\
& <\frac{\varepsilon}{16}, \quad w \geq T_{4} .
\end{aligned}
$$

Combining (15), (22), (28), and (29), we infer that

$$
\begin{aligned}
& \left\|G_{L} y^{w}-G_{L} y\right\| \\
& =\sup \left\{\frac{\left|\left(G_{L} y^{w}\right)(n)-\left(G_{L} y\right)(n)\right|}{n}: n \in \mathbb{N}_{\beta}\right\} \\
& =\max \left\{\sup \left\{\frac{n}{T} \cdot \frac{\left|\left(G_{L} y^{w}\right)(T)-\left(G_{L} y\right)(T)\right|}{n}: \beta \leq n<T\right\},\right. \\
& \left.\sup \left\{\frac{\left|\left(G_{L} y^{w}\right)(n)-\left(G_{L} y\right)(n)\right|}{n}: n \in \mathbb{N}_{T}\right\}\right\}
\end{aligned}
$$$$
=\sup \left\{\frac{\left|\left(G_{L} y^{w}\right)(n)-\left(G_{L} y\right)(n)\right|}{n}: n \in \mathbb{N}_{T}\right\}
$$$$
\leq \frac{1}{T} \sum_{i=T}^{\infty} \mid h\left(i, y^{w}\left(h_{1}(i)\right), \ldots, y^{w}\left(h_{k}(i)\right)\right)
$$$$
-h\left(i, y\left(h_{1}(i)\right), \ldots, y\left(h_{k}(i)\right)\right)
$$$$
+\frac{1}{T} \sum_{i=T}^{\infty} \sum_{s=i}^{\infty} \mid g\left(s, y^{w}\left(g_{1}(s)\right), \ldots, y^{w}\left(g_{k}(s)\right)\right)
$$$$
-g\left(s, y\left(g_{1}(s)\right), \ldots, y\left(g_{k}(s)\right)\right) \mid
$$

$$
\begin{array}{r}
+\frac{1}{T} \sum_{i=T}^{\infty} \sum_{s=i}^{\infty} \sum_{t=s}^{\infty} \mid f\left(t, y^{w}\left(f_{1}(t)\right), \ldots, y^{w}\left(f_{k}(t)\right)\right) \\
-f\left(t, y\left(f_{1}(t)\right), \ldots, y\left(f_{k}(t)\right)\right) \mid
\end{array}
$$$$
=\frac{1}{T} \sum_{i=T}^{T_{1}} \mid h\left(i, y^{w}\left(h_{1}(i)\right), \ldots, y^{w}\left(h_{k}(i)\right)\right)
$$

$$
-h\left(i, y\left(h_{1}(i)\right), \ldots, y\left(h_{k}(i)\right)\right) \mid
$$$$
+\frac{1}{T} \sum_{i=T_{1}+1}^{\infty} \mid h\left(i, y^{w}\left(h_{1}(i)\right), \ldots, y^{w}\left(h_{k}(i)\right)\right)
$$$$
-h\left(i, y\left(h_{1}(i)\right), \ldots, y\left(h_{k}(i)\right)\right) \mid
$$$$
+\frac{1}{T} \sum_{i=T}^{T_{1}} \sum_{s=i}^{T_{2}} \mid g\left(s, y^{w}\left(g_{1}(s)\right), \ldots, y^{w}\left(g_{k}(s)\right)\right)
$$$$
-g\left(s, y\left(g_{1}(s)\right), y\left(g_{2}(s)\right), \ldots, y\left(g_{k}(s)\right)\right) \mid
$$

$+\frac{1}{T} \sum_{i=T}^{T_{1}} \sum_{s=T_{2}+1}^{\infty} \mid g\left(s, y^{w}\left(g_{1}(s)\right), \ldots, y^{w}\left(g_{k}(s)\right)\right)$

$$
-g\left(s, y\left(g_{1}(s)\right), \ldots, y\left(g_{k}(s)\right)\right)
$$




$$
\begin{aligned}
& +\frac{1}{T} \sum_{i=T_{1}+1}^{\infty} \sum_{s=i}^{\infty} \mid g\left(s, y^{w}\left(g_{1}(s)\right), \ldots, y^{w}\left(g_{k}(s)\right)\right) \\
& -g\left(s, y\left(g_{1}(s)\right), \ldots, y\left(g_{k}(s)\right)\right) \mid \\
& +\frac{1}{T} \sum_{i=T}^{T_{1}} \sum_{s=i}^{T_{2}} \sum_{t=s}^{T_{3}} \mid f\left(t, y^{w}\left(f_{1}(t)\right), \ldots, y^{w}\left(f_{k}(t)\right)\right) \\
& -f\left(t, y\left(f_{1}(t)\right), \ldots, y\left(f_{k}(t)\right)\right) \mid \\
& +\frac{1}{T} \sum_{i=T}^{T_{1}} \sum_{s=i}^{T_{2}} \sum_{t=T_{3}+1}^{\infty} \mid f\left(t, y^{w}\left(f_{1}(t)\right), \ldots, y^{w}\left(f_{k}(t)\right)\right) \\
& -f\left(t, y\left(f_{1}(t)\right), \ldots, y\left(f_{k}(t)\right)\right) \\
& +\frac{1}{T} \sum_{i=T}^{T_{1}} \sum_{s=T_{2}+1}^{\infty} \sum_{t=s}^{\infty} \mid f\left(t, y^{w}\left(f_{1}(t)\right), \ldots, y^{w}\left(f_{k}(t)\right)\right) \\
& -f\left(t, y\left(f_{1}(t)\right), \ldots, y\left(f_{k}(t)\right)\right) \mid \\
& +\frac{1}{T} \sum_{i=T_{1}+1}^{\infty} \sum_{s=i}^{\infty} \sum_{t=s}^{\infty} \mid f\left(t, y^{w}\left(f_{1}(t)\right), \ldots, y^{w}\left(f_{k}(t)\right)\right) \\
& -f\left(t, y\left(f_{1}(t)\right), \ldots, y\left(f_{k}(t)\right)\right) \mid \\
& <\frac{\varepsilon}{16}+\frac{2}{T} \sum_{i=T_{1}+1}^{\infty} H_{i}+\frac{\varepsilon}{16}+\frac{2}{T} \sum_{i=T}^{T_{1}} \sum_{s=T_{2}+1}^{\infty} G_{s} \\
& +\frac{2}{T} \sum_{i=T_{1}+1}^{\infty} \sum_{s=i}^{\infty} G_{s}+\frac{\varepsilon}{16}+\frac{2}{T} \sum_{i=T}^{T_{1}} \sum_{s=i}^{T_{2}} \sum_{t=T_{3}+1}^{\infty} F_{t} \\
& +\frac{2}{T} \sum_{i=T}^{T_{1}} \sum_{s=T_{2}+1}^{\infty} \sum_{t=s}^{\infty} F_{t} \\
& +\frac{2}{T} \sum_{i=T_{1}+1}^{\infty} \sum_{s=i}^{\infty} \sum_{t=s}^{\infty} F_{t}<\varepsilon, \quad w \geq T_{4},
\end{aligned}
$$

which implies that $G_{L}$ is continuous in $\Omega\left(A_{*}, B^{*}, T\right)$.

It follows from (15), (22), and (28) that for any $x=$ $\{x(n)\}_{n \in \mathbb{N}_{\beta}} \in \Omega\left(A_{*}, B^{*}, T\right)$ and $t_{1}, t_{2} \geq T_{4}$

$$
\begin{aligned}
& \left|\frac{\left(G_{L} y\right)\left(t_{2}\right)}{t_{2}}-\frac{\left(G_{L} y\right)\left(t_{1}\right)}{t_{1}}\right| \\
& =\mid \frac{1}{t_{2}} \sum_{i=t_{2}}^{\infty} h\left(i, y\left(h_{1}(i)\right), \ldots, y\left(h_{k}(i)\right)\right) \\
& -\frac{1}{t_{1}} \sum_{i=t_{1}}^{\infty} h\left(i, y\left(h_{1}(i)\right), \ldots, y\left(h_{k}(i)\right)\right)
\end{aligned}
$$

$$
\begin{aligned}
& -\frac{1}{t_{2}} \sum_{i=t_{2}}^{\infty} \sum_{s=i}^{\infty} g\left(s, y\left(g_{1}(s)\right), \ldots, y\left(g_{k}(s)\right)\right) \\
& +\frac{1}{t_{1}} \sum_{i=t_{1}}^{\infty} \sum_{s=i}^{\infty} g\left(s, y\left(g_{1}(s)\right), \ldots, y\left(g_{k}(s)\right)\right) \\
& +\frac{1}{t_{2}} \sum_{i=t_{2}}^{\infty} \sum_{s=i}^{\infty} \sum_{t=s}^{\infty}\left[f\left(t, y\left(f_{1}(t)\right), \ldots, y\left(f_{k}(t)\right)\right)-d(t)\right] \\
& -\frac{1}{t_{1}} \sum_{i=t_{1}}^{\infty} \sum_{s=i}^{\infty} \sum_{t=s}^{\infty}\left[f\left(t, y\left(f_{1}(t)\right), \ldots, y\left(f_{k}(t)\right)\right)-d(t)\right] \\
& \leq \frac{2}{T_{4}}\left(\sum_{i=T_{4}}^{\infty} H_{i}+\sum_{i=T_{4}}^{\infty} \sum_{s=i}^{\infty} G_{s}+\sum_{i=T_{4}}^{\infty} \sum_{s=i}^{\infty} \sum_{t=s}^{\infty}\left[F_{t}+|d(t)|\right]\right) \\
& <\varepsilon,
\end{aligned}
$$

which means that $G_{L}\left(\Omega\left(A_{*}, B^{*}, T\right)\right)$ is uniformly Cauchy, which together with $(25)$ and Lemma 1 yields that $G_{L}\left(\Omega\left(A_{*}\right.\right.$, $\left.B^{*}, T\right)$ ) is relatively compact. Hence $G_{L}$ is completely continuous in $\Omega\left(A_{*}, B^{*}, T\right)$. Thus (14), (24), and Lemma 2 ensure that the mapping $S_{L}+G_{L}$ has a fixed point $x=\{x(n)\}_{n \in \mathbb{N}_{\beta}}$, which together with (21) and (22) implies that

$$
\begin{aligned}
x(n) & \\
= & n L-b(n) x(n-\tau)-c(n) \\
& +\sum_{i=n}^{\infty} h\left(i, x\left(h_{1}(i)\right), \ldots, x\left(h_{k}(i)\right)\right) \\
& -\sum_{i=n}^{\infty} \sum_{s=i}^{\infty} g\left(s, x\left(g_{1}(s)\right), \ldots, x\left(g_{k}(s)\right)\right) \\
& +\sum_{i=n}^{\infty} \sum_{s=i}^{\infty} \sum_{t=s}^{\infty}\left[f\left(t, x\left(f_{1}(t)\right), \ldots, x\left(f_{k}(t)\right)\right)-d(t)\right],
\end{aligned}
$$

which gives that

$$
\begin{aligned}
& \Delta(x(n)+b(n) x(n-\tau)+c(n)) \\
& =L-h\left(n, x\left(h_{1}(n)\right), \ldots, x\left(h_{k}(n)\right)\right) \\
& \quad+\sum_{s=n}^{\infty} g\left(s, x\left(g_{1}(s)\right), \ldots, x\left(g_{k}(s)\right)\right) \\
& \quad-\sum_{s=n}^{\infty} \sum_{t=s}^{\infty}\left[f\left(t, x\left(f_{1}(t)\right), \ldots, x\left(f_{k}(t)\right)\right)-d(t)\right], \\
& \quad n \geq T+\tau,
\end{aligned}
$$




$$
\begin{aligned}
& \Delta^{2}(x(n)+b(n) x(n-\tau)+c(n)) \\
&=-\Delta h\left(n, x\left(h_{1}(n)\right), \ldots, x\left(h_{k}(n)\right)\right) \\
&-g\left(n, x\left(g_{1}(n)\right), \ldots, x\left(g_{k}(n)\right)\right) \\
&+\sum_{t=n}^{\infty}\left[f\left(t, x\left(f_{1}(t)\right), \ldots, x\left(f_{k}(t)\right)\right)-d(t)\right], \\
& \quad n \geq T+\tau, \\
& \Delta^{3}(x(n)+b(n) x(n-\tau)+c(n)) \quad \\
&=-\Delta^{2} h\left(n, x\left(h_{1}(n)\right), \ldots, x\left(h_{k}(n)\right)\right) \\
& \quad-\Delta g\left(n, x\left(g_{1}(n)\right), \ldots, x\left(g_{k}(n)\right)\right) \\
& \quad-\left[f\left(n, x\left(f_{1}(n)\right), \ldots, x\left(f_{k}(n)\right)\right)-d(n)\right], \\
& n \geq T+\tau,
\end{aligned}
$$

that is, $x=\{x(n)\}_{n \in \mathbb{N}_{\beta}} \in \Omega\left(A_{*}, B^{*}, T\right)$ is a positive solution of (4). It follows from (15)-(17), and (32) that

$$
\begin{aligned}
&\left|\frac{x(n)+b(n) x(n-\tau)+c(n)}{n}-L\right| \\
&=\mid \frac{1}{n} \sum_{i=n}^{\infty} h\left(i, x\left(h_{1}(i)\right), \ldots, x\left(h_{k}(i)\right)\right) \\
& \quad-\frac{1}{n} \sum_{i=n}^{\infty} \sum_{s=i}^{\infty} g\left(s, x\left(g_{1}(s)\right), \ldots, x\left(g_{k}(s)\right)\right) \\
&+\frac{1}{n} \sum_{i=n}^{\infty} \sum_{s=i}^{\infty} \sum_{t=s}^{\infty}\left[f\left(t, x\left(f_{1}(t)\right), \ldots, x\left(f_{k}(t)\right)\right)-d(t)\right] \mid \\
& \leq \frac{1}{n} \sum_{i=n}^{\infty} H_{i}+\frac{1}{n} \sum_{i=n}^{\infty} \sum_{s=i}^{\infty} G_{s}+\frac{1}{n} \sum_{i=n}^{\infty} \sum_{s=i}^{\infty} \sum_{t=s}^{\infty} F_{t} \longrightarrow 0 \\
& A_{*}= A-c^{*} \leq A-\frac{|c(n)|}{n} \\
&= A(n) \leq \frac{x(n)}{n} \leq B(n)=B+\frac{|c(n)|}{n} \\
& \leq B+c^{*}=B^{*}, \quad n \geq T,
\end{aligned}
$$

which yield that

$$
\begin{gathered}
\lim _{n \rightarrow \infty} \frac{x(n)+b(n) x(n-\tau)+c(n)}{n}=L \in\left(A+b^{*} B^{*}, B\right), \\
A_{*} \leq \liminf _{n \rightarrow \infty} \frac{x(n)}{n} \leq \limsup _{n \rightarrow \infty} \frac{x(n)}{n} \leq B^{*} ;
\end{gathered}
$$

that is, (18) and (19) hold. (ii) Let $L_{1}, L_{2} \in\left(A+b^{*} B^{*}, B\right)$ and $L_{1} \neq L_{2}$. As in the proof of (i), we infer that for each $l \in\{1,2\}$, there exist a constant $T_{l} \geq n_{0}+\tau+\alpha$ and two mappings $S_{L_{l}}$ and $G_{L_{l}}: \Omega\left(A_{*}, B^{*}\right.$, $\left.T_{l}\right) \rightarrow l_{\beta}^{\infty}$ satisfying (20)-(22), where $T, L, S_{L}$ and $G_{L}$ are replaced by $T_{l}, L_{l}, S_{L_{l}}$ and $G_{L_{l}}$, respectively, and $S_{L_{l}}+G_{L_{l}}$ possesses a fixed point $x_{l}=\left\{x_{l}(n)\right\}_{n \in \mathbb{N}_{\beta}}$, which is a positive solution of (4); that is,

$x_{l}(n)=n L_{l}-b(n) x_{l}(n-\tau)-c(n)$

$$
\begin{aligned}
& +\sum_{i=n}^{\infty} h\left(i, x_{l}\left(h_{1}(i)\right), \ldots, x_{l}\left(h_{k}(i)\right)\right) \\
& -\sum_{i=n}^{\infty} \sum_{s=i}^{\infty} g\left(s, x_{l}\left(g_{1}(s)\right), \ldots, x_{l}\left(g_{k}(s)\right)\right) \\
& +\sum_{i=n}^{\infty} \sum_{s=i}^{\infty} \sum_{t=s}^{\infty}\left[f\left(t, x_{l}\left(f_{1}(t)\right), \ldots, x_{l}\left(f_{k}(t)\right)\right)-d(t)\right],
\end{aligned}
$$

Note that (16) and (17) imply that there exists $T_{*}>\max \left\{T_{1}\right.$, $\left.\mathrm{T}_{2}\right\}$ satisfying

$$
\begin{gathered}
\frac{1}{T_{*}}\left(\sum_{i=T_{*}}^{\infty} H_{i}+\sum_{i=T_{*}}^{\infty} \sum_{s=i}^{\infty} G_{s}+\sum_{i=T_{*}}^{\infty} \sum_{s=i}^{\infty} \sum_{t=s}^{\infty} F_{t}\right) \\
<\frac{\left|L_{1}-L_{2}\right|}{4},
\end{gathered}
$$

which together with (15) and (36) means that for any $n \geq T_{*}$

$$
\begin{aligned}
& \left|\frac{x_{1}(n)}{n}-\frac{x_{2}(n)}{n}\right| \\
& =\mid L_{1}-L_{2}-\frac{b(n)}{n}\left(x_{1}(n-\tau)-x_{2}(n-\tau)\right)
\end{aligned}
$$$$
+\frac{1}{n} \sum_{i=n}^{\infty}\left[h\left(i, x_{1}\left(h_{1}(i)\right), \ldots, x_{1}\left(h_{k}(i)\right)\right)\right.
$$$$
\left.-h\left(i, x_{2}\left(h_{1}(i)\right), \ldots, x_{2}\left(h_{k}(i)\right)\right)\right]
$$$$
-\frac{1}{n} \sum_{i=n}^{\infty} \sum_{s=i}^{\infty}\left[g\left(s, x_{1}\left(g_{1}(s)\right), \ldots, x_{1}\left(g_{k}(s)\right)\right)\right.
$$

$$
\left.-g\left(s, x_{2}\left(g_{1}(s)\right), \ldots, x_{2}\left(g_{k}(s)\right)\right)\right]
$$

$$
+\frac{1}{n} \sum_{i=n}^{\infty} \sum_{s=i}^{\infty} \sum_{t=s}^{\infty}\left[f\left(t, x_{1}\left(f_{1}(t)\right), \ldots, x_{1}\left(f_{k}(t)\right)\right)\right.
$$

$$
\left.-f\left(t, x_{2}\left(f_{1}(t)\right), \ldots, x_{2}\left(f_{k}(t)\right)\right)\right]
$$




$$
\begin{aligned}
& \geq\left|L_{1}-L_{2}\right| \\
& -\frac{(n-\tau) b(n)}{n}\left|\frac{x_{1}(n-\tau)-x_{2}(n-\tau)}{n-\tau}\right| \\
& -\frac{1}{T_{*}} \sum_{i=T_{*}}^{\infty} \mid h\left(i, x_{1}\left(h_{1}(i)\right), \ldots, x_{1}\left(h_{k}(i)\right)\right) \\
& -h\left(i, x_{2}\left(h_{1}(i)\right), \ldots, x_{2}\left(h_{k}(i)\right)\right) \mid \\
& -\frac{1}{T_{*}} \sum_{i=T_{*}}^{\infty} \sum_{s=i}^{\infty} \mid g\left(s, x_{1}\left(g_{1}(s)\right), \ldots, x_{1}\left(g_{k}(s)\right)\right) \\
& -g\left(s, x_{2}\left(g_{1}(s)\right), \ldots, x_{2}\left(g_{k}(s)\right)\right) \mid \\
& -\frac{1}{T_{*}} \sum_{i=T_{*}}^{\infty} \sum_{s=i}^{\infty} \sum_{t=s}^{\infty} \mid f\left(t, x_{1}\left(f_{1}(t)\right), \ldots, x_{1}\left(f_{k}(t)\right)\right) \\
& -f\left(t, x_{2}\left(f_{1}(t)\right), \ldots, x_{2}\left(f_{k}(t)\right)\right) \mid \\
& \geq\left|L_{1}-L_{2}\right|-b^{*}\left\|x_{1}-x_{2}\right\| \\
& -\frac{2}{T_{*}}\left(\sum_{i=T_{*}}^{\infty} H_{i}+\sum_{i=T_{*}}^{\infty} \sum_{s=i}^{\infty} G_{s}+\sum_{i=T_{*}}^{\infty} \sum_{s=i}^{\infty} \sum_{t=s}^{\infty} F_{t}\right) \\
& >\frac{\left|L_{1}-L_{2}\right|}{2}-b^{*}\left\|x_{1}-x_{2}\right\|,
\end{aligned}
$$

which implies that

$$
\left\|x_{1}-x_{2}\right\|>\frac{\left|L_{1}-L_{2}\right|}{2\left(1+b^{*}\right)}>0,
$$

which yields that $x_{1} \neq x_{2}$. Therefore (4) possesses uncountably many positive solutions in $l_{\beta}^{\infty}$. This completes the proof.

Theorem 6. Assume that there exist constants $b_{*}$ and $b^{*}$ and three nonnegative sequences $\left\{F_{n}\right\}_{n \in \mathbb{N}_{n_{0}}},\left\{G_{n}\right\}_{n \in \mathbb{N}_{n_{0}}}$, and $\left\{H_{n}\right\}_{n \in \mathbb{N}_{n_{0}}}$ satisfying (15)-(17) and

$$
\begin{array}{r}
b^{*} A+\left(B^{*}+c^{*}\right) \frac{b^{*}}{b_{*}}<b_{*} B+\frac{b_{*} A_{*}}{b^{*}}-c^{*}, \\
1<b_{*} \leq b(n) \leq b^{*}, \quad n \in \mathbb{N}_{n_{0}} .
\end{array}
$$

Then

(i) equation (4) possesses a positive solution $x=$ $\{x(n)\}_{n \in \mathbb{N}_{\beta}} \in l_{\beta}^{\infty}$ with (19) and

$$
\begin{aligned}
\lim _{n \rightarrow \infty} & \frac{x(n)+b(n) x(n-\tau)+c(n)}{n} \\
& \in\left(b^{*} A+\left(B^{*}+c^{*}\right) \frac{b^{*}}{b_{*}}, b_{*} B+\frac{b_{*} A_{*}}{b^{*}}-c^{*}\right) ;
\end{aligned}
$$

(ii) equation (4) possesses uncountably many positive solutions in $l_{\beta}^{\infty}$.

Proof. (i) Put $L \in\left(b^{*} A+\left(B^{*}+c^{*}\right)\left(b^{*} / b_{*}\right), b_{*} B+b_{*} A_{*} / b^{*}-c^{*}\right)$. Observe that

$$
\begin{gathered}
\lim _{n \rightarrow \infty}\left[b^{*} A+\left(1+\frac{\tau}{n}\right)\left(B^{*}+c^{*}\right) \frac{b^{*}}{b_{*}}\right] \\
=b^{*} A+\left(B^{*}+c^{*}\right) \frac{b^{*}}{b_{*}}<L<b_{*} B+\frac{b_{*} A_{*}}{b^{*}}-c^{*} \\
=\lim _{n \rightarrow \infty}\left[b_{*} B+\frac{b_{*} A_{*}}{b^{*}}-c^{*}\left(1+\frac{\tau}{n}\right)\right],
\end{gathered}
$$

which yields that there exists $N \in \mathbb{N}$ satisfying

$$
\begin{aligned}
b^{*} A+\left(B^{*}+c^{*}\right) \frac{b^{*}}{b_{*}} & <b^{*} A+\left(1+\frac{\tau}{N}\right)\left(B^{*}+c^{*}\right) \frac{b^{*}}{b_{*}} \\
& <L<b_{*} B+\frac{b_{*} A_{*}}{b^{*}}-c^{*}\left(1+\frac{\tau}{N}\right) \\
& <b_{*} B+\frac{b_{*} A_{*}}{b^{*}}-c^{*}
\end{aligned}
$$

It follows from (16) and (17) that there exist $\theta \in(0,1)$ and $T>\max \left\{n_{0}+\tau+\alpha, N\right\}$ satisfying

$$
\theta=\frac{1}{b_{*}}\left(1+\frac{\tau}{T}\right)
$$

$$
\begin{array}{r}
\frac{1}{T} \sum_{i=T+\tau}^{\infty}\left\{H_{i}+\sum_{s=i}^{\infty} G_{s}+\sum_{s=i}^{\infty} \sum_{t=s}^{\infty}\left[F_{t}+|d(t)|\right]\right\} \\
<\min \left\{b_{*} B+\frac{b_{*} A_{*}}{b^{*}}-c^{*}\left(1+\frac{\tau}{N}\right)-L,\right. \\
\left.\frac{b_{*} L}{b^{*}}-b_{*} A-\left(1+\frac{\tau}{N}\right)\left(B^{*}+c^{*}\right)\right\} \\
<\min \left\{b_{*} B+\frac{b_{*} A_{*}}{b^{*}}-c^{*}\left(1+\frac{\tau}{T}\right)-L,\right. \\
\left.\frac{b_{*} L}{b^{*}}-b_{*} A-\left(1+\frac{\tau}{T}\right)\left(B^{*}+c^{*}\right)\right\} .
\end{array}
$$

Define two mappings $S_{L}$ and $G_{L}: \Omega\left(A_{*}, B^{*}, T\right) \rightarrow l_{\beta}^{\infty}$ by $\left(S_{L} x\right)(n)$

$$
= \begin{cases}\frac{n L}{b(n+\tau)}-\frac{x(n+\tau)}{b(n+\tau)}-\frac{c(n+\tau)}{b(n+\tau)}, & n \geq T, \\ \frac{n}{T}\left(S_{L} x\right)(T), & \beta \leq n<T,\end{cases}
$$




$$
\begin{aligned}
& \left(G_{L} x\right)(n) \\
& =\left\{\begin{array}{c}
\frac{1}{b(n+\tau)} \sum_{i=n+\tau}^{\infty} h\left(i, x\left(h_{1}(i)\right), \ldots, x\left(h_{k}(i)\right)\right) \\
-\frac{1}{b(n+\tau)} \sum_{i=n+\tau}^{\infty} \sum_{s=i}^{\infty} g\left(s, x\left(g_{1}(s)\right), \ldots, x\left(g_{k}(s)\right)\right) \\
+\frac{1}{b(n+\tau)} \\
\cdot \sum_{i=n+\tau}^{\infty} \sum_{s=i}^{\infty} \sum_{t=s}^{\infty}\left[f\left(t, x\left(f_{1}(t)\right), \ldots, x\left(f_{k}(t)\right)\right)\right. \\
\frac{n}{T}\left(G_{L} x\right)(T), \quad n \geq T, \\
\end{array}\right.
\end{aligned}
$$

for any $x=\{x(n)\}_{n \in \mathbb{N}_{\beta}} \in \Omega\left(A_{*}, B^{*}, T\right)$.

Now we show that (23), (48), and (49) below hold

$\left\|S_{L} x-S_{L} y\right\| \leq \theta\|x-y\|, \quad x, y \in \Omega\left(A_{*}, B^{*}, T\right) ;$

$$
\left\|G_{L} y\right\| \leq B+\frac{A_{*}}{b^{*}}, \quad y \in \Omega\left(A_{*}, B^{*}, T\right) .
$$

Using (15) and (44)-(47), we get that for any $x=$ $\{x(n)\}_{n \in \mathbb{N}_{\beta}}, y=\{y(n)\}_{n \in \mathbb{N}_{\beta}} \in \Omega\left(A_{*}, B^{*}, T\right)$

$$
\begin{aligned}
& \frac{\left(S_{L} x\right)(n)+\left(G_{L} y\right)(n)}{n} \\
& =\frac{L}{b(n+\tau)}-\frac{x(n+\tau)}{n b(n+\tau)}-\frac{c(n+\tau)}{n b(n+\tau)} \\
& \quad+\frac{1}{n b(n+\tau)} \sum_{i=n+\tau}^{\infty} h\left(i, y\left(h_{1}(i)\right), \ldots, y\left(h_{k}(i)\right)\right) \\
& \quad-\frac{1}{n b(n+\tau)} \sum_{i=n+\tau}^{\infty} \sum_{s=i}^{\infty} g\left(s, y\left(g_{1}(s)\right), \ldots, y\left(g_{k}(s)\right)\right) \\
& \quad+\frac{1}{n b(n+\tau)} \\
& \quad \cdot \sum_{i=n+\tau}^{\infty} \sum_{s=i}^{\infty} \sum_{t=s}^{\infty}\left[f\left(t, y\left(f_{1}(t)\right), \ldots, y\left(f_{k}(t)\right)\right)-d(t)\right] \\
& \leq \frac{L}{b_{*}}-\frac{x(n+\tau)}{(n+\tau) b(n+\tau)}+\frac{n+\tau}{n b(n+\tau)} \\
& \quad \cdot \frac{|c(n+\tau)|}{n+\tau}+\frac{1}{n b(n+\tau)} \\
& \quad \cdot \sum_{i=n+\tau}^{\infty}\left|h\left(i, y\left(h_{1}(i)\right), \ldots, y\left(h_{k}(i)\right)\right)\right|+\frac{1}{n b(n+\tau)}
\end{aligned}
$$

$$
\begin{aligned}
& \cdot \sum_{i=n+\tau}^{\infty} \sum_{s=i}^{\infty}\left|g\left(s, y\left(g_{1}(s)\right), \ldots, y\left(g_{k}(s)\right)\right)\right| \\
& +\frac{1}{n b(n+\tau)} \\
& \cdot \sum_{i=n+\tau}^{\infty} \sum_{s=i}^{\infty} \sum_{t=s}^{\infty}\left[\left|f\left(t, y\left(f_{1}(t)\right), \ldots, y\left(f_{k}(t)\right)\right)\right|+|d(t)|\right] \\
& \leq \frac{L}{b_{*}}-\frac{A_{*}}{b^{*}}+\frac{c^{*}}{b_{*}}\left(1+\frac{\tau}{T}\right)+\frac{1}{T b_{*}} \sum_{i=T+\tau}^{\infty} H_{i} \\
& +\frac{1}{T b_{*}} \sum_{i=T+\tau}^{\infty} \sum_{s=i}^{\infty} G_{s}+\frac{1}{T b_{*}} \sum_{i=T+\tau}^{\infty} \sum_{s=i}^{\infty} \sum_{t=s}^{\infty}\left[F_{t}+|d(t)|\right] \\
& <\frac{L}{b_{*}}-\frac{A_{*}}{b^{*}}+\frac{c^{*}}{b_{*}}\left(1+\frac{\tau}{T}\right) \\
& +\frac{1}{b_{*}} \min \left\{b_{*} B+\frac{b_{*} A_{*}}{b^{*}}-c^{*}\left(1+\frac{\tau}{T}\right)-L,\right. \\
& \left.\frac{b_{*}}{b^{*}} L-b_{*} A-\left(1+\frac{\tau}{T}\right)\left(B^{*}+c^{*}\right)\right\}
\end{aligned}
$$$$
\leq B \leq B(n), \quad n \geq T,
$$$$
\frac{\left(S_{L} x\right)(n)+\left(G_{L} y\right)(n)}{n}=\frac{n}{T} \cdot \frac{\left(S_{L} x\right)(T)+\left(G_{L} y\right)(T)}{n}
$$$$
\leq B(T), \quad \beta \leq n<T,
$$$$
\frac{\left(S_{L} x\right)(n)+\left(G_{L} y\right)(n)}{n}
$$$$
=\frac{L}{b(n+\tau)}-\frac{x(n+\tau)}{n b(n+\tau)}-\frac{c(n+\tau)}{n b(n+\tau)}
$$$$
+\frac{1}{n b(n+\tau)} \sum_{i=n+\tau}^{\infty} h\left(i, y\left(h_{1}(i)\right), \ldots, y\left(h_{k}(i)\right)\right)
$$$$
-\frac{1}{n b(n+\tau)} \sum_{i=n+\tau}^{\infty} \sum_{s=i}^{\infty} g\left(s, y\left(g_{1}(s)\right), \ldots, y\left(g_{k}(s)\right)\right)
$$$$
+\frac{1}{n b(n+\tau)}
$$$$
\cdot \sum_{i=n+\tau}^{\infty} \sum_{s=i}^{\infty} \sum_{t=s}^{\infty}\left[f\left(t, y\left(f_{1}(t)\right), \ldots, y\left(f_{k}(t)\right)\right)-d(t)\right]
$$$$
\geq \frac{L}{b^{*}}-\frac{n+\tau}{n b(n+\tau)} \cdot \frac{x(n+\tau)}{n+\tau}-\frac{n+\tau}{n b(n+\tau)}
$$$$
\cdot \frac{|c(n+\tau)|}{n+\tau}-\frac{1}{n b(n+\tau)}
$$ 


$$
\begin{aligned}
& \cdot \sum_{i=n+\tau}^{\infty}\left|h\left(i, y\left(h_{1}(i)\right), \ldots, y\left(h_{k}(i)\right)\right)\right| \\
& -\frac{1}{n b(n+\tau)} \sum_{i=n+\tau}^{\infty} \sum_{s=i}^{\infty}\left|g\left(s, y\left(g_{1}(s)\right), \ldots, y\left(g_{k}(s)\right)\right)\right| \\
& -\frac{1}{n b(n+\tau)} \\
& \cdot \sum_{i=n+\tau}^{\infty} \sum_{s=i}^{\infty} \sum_{t=s}^{\infty}\left[\left|f\left(t, y\left(f_{1}(t)\right), \ldots, y\left(f_{k}(t)\right)\right)\right|+|d(t)|\right] \\
& \geq \frac{L}{b^{*}}-\frac{1}{b_{*}}\left(1+\frac{\tau}{T}\right) B^{*}-\frac{c^{*}}{b_{*}}\left(1+\frac{\tau}{T}\right) \\
& -\frac{1}{T b_{*}} \sum_{i=T+\tau}^{\infty} H_{i}-\frac{1}{T b_{*}} \sum_{i=T+\tau}^{\infty} \sum_{s=i}^{\infty} G_{s}-\frac{1}{T b_{*}} \\
& \cdot \sum_{i=T+\tau}^{\infty} \sum_{s=i}^{\infty} \sum_{t=s}^{\infty}\left[F_{t}+|d(t)|\right] \\
& >\frac{L}{b^{*}}-\frac{1}{b_{*}}\left(1+\frac{\tau}{T}\right)\left(B^{*}+c^{*}\right) \\
& -\frac{1}{b_{*}} \min \left\{b_{*} B+\frac{b_{*} A_{*}}{b^{*}}-c^{*}\left(1+\frac{\tau}{T}\right)-L,\right. \\
& \left.\frac{b_{*}}{b^{*}} L-b_{*} A-\left(1+\frac{\tau}{T}\right)\left(B^{*}+c^{*}\right)\right\} \\
& \geq A \geq A(n), \quad n \geq T, \\
& \begin{aligned}
\frac{\left(S_{L} x\right)(n)+\left(G_{L} y\right)(n)}{n} & =\frac{n}{T} \cdot \frac{\left(S_{L} x\right)(T)+\left(G_{L} y\right)(T)}{n} \\
& \geq A(T), \quad \beta \leq n<T
\end{aligned} \\
& \left|\frac{\left(S_{L} x\right)(n)-\left(S_{L} y\right)(n)}{n}\right|=\frac{n+\tau}{n b(n+\tau)}\left|\frac{x(n+\tau)-y(n+\tau)}{n+\tau}\right| \\
& \leq \theta\|x-y\|, \quad n \geq T, \\
& +\frac{1}{n b(n+\tau)} \\
& \cdot \sum_{i=n+\tau}^{\infty} \sum_{s=i}^{\infty} \sum_{t=s}^{\infty}\left[f\left(t, y\left(f_{1}(t)\right), \ldots, y\left(f_{k}(t)\right)\right)-d(t)\right] \\
& \leq \frac{1}{n b(n+\tau)} \\
& \cdot \sum_{i=n+\tau}^{\infty}\left|h\left(i, y\left(h_{1}(i)\right), \ldots, y\left(h_{k}(i)\right)\right)\right| \\
& +\frac{1}{n b(n+\tau)} \\
& \cdot \sum_{i=n+\tau}^{\infty} \sum_{s=i}^{\infty}\left|g\left(s, y\left(g_{1}(s)\right), \ldots, y\left(g_{k}(s)\right)\right)\right| \\
& +\frac{1}{n b(n+\tau)} \\
& \cdot \sum_{i=n+\tau}^{\infty} \sum_{s=i}^{\infty} \sum_{t=s}^{\infty}\left[\left|f\left(t, y\left(f_{1}(t)\right), \ldots, y\left(f_{k}(t)\right)\right)\right|+|d(t)|\right] \\
& \leq \frac{1}{T b_{*}} \sum_{i=T+\tau}^{\infty} H_{i}+\frac{1}{T b_{*}} \sum_{i=T+\tau}^{\infty} \sum_{s=i}^{\infty} G_{s} \\
& +\frac{1}{T b_{*}} \sum_{i=T+\tau}^{\infty} \sum_{s=i}^{\infty} \sum_{t=s}^{\infty}\left[F_{t}+|d(t)|\right] \\
& <\frac{1}{b_{*}} \min \left\{b_{*} B+\frac{b_{*} A_{*}}{b^{*}}-c^{*}\left(1+\frac{\tau}{T}\right)-L,\right. \\
& \left.\frac{b_{*}}{b^{*}} L-b_{*} A-\left(1+\frac{\tau}{T}\right)\left(B^{*}+c^{*}\right)\right\} \\
& \leq B+\frac{A_{*}}{b^{*}}, \quad n \geq T, \\
& \left|\frac{\left(G_{L} y\right)(n)}{n}\right|=\frac{n}{T}\left|\frac{\left(G_{L} y\right)(T)}{n}\right| \leq B+\frac{A_{*}}{b^{*}}, \quad \beta \leq n<T,
\end{aligned}
$$$$
\left|\frac{\left(S_{L} x\right)(n)-\left(S_{L} y\right)(n)}{n}\right|=\frac{n}{T}\left|\frac{\left(S_{L} x\right)(T)-\left(S_{L} y\right)(T)}{n}\right|
$$$$
\leq \theta\|x-y\|, \quad \beta \leq n<T,
$$$$
\left|\frac{\left(G_{L} y\right)(n)}{n}\right|
$$$$
=\mid \frac{1}{n b(n+\tau)} \sum_{i=n+\tau}^{\infty} h\left(i, y\left(h_{1}(i)\right), \ldots, y\left(h_{k}(i)\right)\right)
$$$$
-\frac{1}{n b(n+\tau)} \sum_{i=n+\tau}^{\infty} \sum_{s=i}^{\infty} g\left(s, y\left(g_{1}(s)\right), \ldots, y\left(g_{k}(s)\right)\right)
$$

which yield (23), (48), and (49).

Next we show that $G_{L}$ is completely continuous. Let $y^{w}=$ $\left\{y^{w}(n)\right\}_{n \in \mathbb{N}_{\beta}}$ and $y=\{y(n)\}_{n \in \mathbb{N}_{\beta}} \in \Omega\left(A_{*}, B^{*}, T\right)$ with (27). Using (16), (17), (27), (47), and the continuity of $f, g$, and $h$, we know that for given $\varepsilon>0$, there exist $T_{1}, T_{2}, T_{3}$, and $T_{4} \in \mathbb{N}$ with $T_{4}>T_{3}>T_{2}>T_{1}>T+\tau$ satisfying

$$
\begin{aligned}
\frac{1}{T b_{*}} \max \left\{\sum_{i=T_{1}+1}^{\infty} H_{i}+\sum_{i=T_{1}+1}^{\infty} \sum_{s=i}^{\infty} G_{s}\right. \\
+\sum_{i=T_{1}+1}^{\infty} \sum_{s=i}^{\infty} \sum_{t=s}^{\infty}\left[F_{t}+|d(t)|\right], \sum_{i=T+\tau}^{T_{1}} \sum_{s=T_{2}+1}^{\infty} G_{s}
\end{aligned}
$$




$$
\begin{aligned}
& \left.+\sum_{i=T+\tau}^{T_{1}} \sum_{s=T_{2}+1}^{\infty} \sum_{t=s}^{\infty} F_{t}+\sum_{i=T+\tau}^{T_{1}} \sum_{s=i}^{T_{2}} \sum_{t=T_{3}+1}^{\infty} F_{t}\right\} \\
& <\frac{\varepsilon}{16}, \\
& \frac{1}{T b_{*}} \max \left\{\sum_{i=T+\tau}^{T_{1}} \mid h\left(i, y^{w}\left(h_{1}(i)\right), \ldots, y^{w}\left(h_{k}(i)\right)\right)\right. \\
& -h\left(i, y\left(h_{1}(i)\right), \ldots, y\left(h_{k}(i)\right)\right) \mid, \\
& \sum_{i=T+\tau s=i}^{T_{1}} \sum^{T_{2}} \mid g\left(s, y^{w}\left(g_{1}(s)\right), \ldots, y^{w}\left(g_{k}(s)\right)\right) \\
& -g\left(s, y\left(g_{1}(s)\right), \ldots, y\left(g_{k}(s)\right)\right) \mid, \\
& \sum_{i=T+\tau}^{T_{1}} \sum_{s=i}^{T_{2}} \sum_{t=s}^{T_{3}} \mid f\left(t, y^{w}\left(f_{1}(t)\right), \ldots, y^{w}\left(f_{k}(t)\right)\right) \\
& \left.-f\left(t, y\left(f_{1}(t)\right), \ldots, y\left(f_{k}(t)\right)\right) \mid\right\} \\
& <\frac{\varepsilon}{16}, \quad w \geq T_{4} .
\end{aligned}
$$

$$
\begin{aligned}
& -f\left(t, y\left(f_{1}(t)\right), \ldots, y\left(f_{k}(t)\right)\right) \mid: \\
& \left.n \in \mathbb{N}_{T}\right\} \\
& +\frac{1}{n b(n+\tau)} \\
& \cdot \sum_{i=n+\tau}^{\infty} \sum_{s=i}^{\infty} \sum_{t=s}^{\infty} \mid f\left(t, y^{w}\left(f_{1}(t)\right), \ldots, y^{w}\left(f_{k}(t)\right)\right)
\end{aligned}
$$$$
\leq \frac{1}{T b_{*}} \sum_{i=T+\tau}^{T_{1}} \mid h\left(i, y^{w}\left(h_{1}(i)\right), \ldots, y^{w}\left(h_{k}(i)\right)\right)
$$

Combining (15), (47), (51), and (52), we infer that

$$
\left\|G_{L} y^{w}-G_{L} y\right\|
$$$$
=\sup \left\{\frac{\left|\left(G_{L} y^{w}\right)(n)-\left(G_{L} y\right)(n)\right|}{n}: n \in \mathbb{N}_{\beta}\right\}
$$$$
=\max \left\{\sup \left\{\frac{n}{T} \cdot \frac{\left|\left(G_{L} y^{w}\right)(T)-\left(G_{L} y\right)(T)\right|}{n}: \beta \leq n<T\right\}\right. \text {, }
$$

$$
\begin{aligned}
\left.\sup \left\{\frac{\left|\left(G_{L} y^{w}\right)(n)-\left(G_{L} y\right)(n)\right|}{n}: n \in \mathbb{N}_{T}\right\}\right\} \\
\leq \sup \left\{\frac{1}{n b(n+\tau)}\right. \\
\cdot \sum_{i=n+\tau}^{\infty} \mid h\left(i, y^{w}\left(h_{1}(i)\right), \ldots, y^{w}\left(h_{k}(i)\right)\right) \\
+\frac{1}{n b(n+\tau)}-h\left(i, y\left(h_{1}(i)\right), \ldots, y\left(h_{k}(i)\right)\right) \mid \\
\cdot \sum_{i=n+\tau}^{\infty} \sum_{s=i}^{\infty} \mid g\left(s, y^{w}\left(g_{1}(s)\right), \ldots, y^{w}\left(g_{k}(s)\right)\right) \\
-g\left(s, y\left(g_{1}(s)\right), \ldots, y\left(g_{k}(s)\right)\right) \mid
\end{aligned}
$$$$
-h\left(i, y\left(h_{1}(i)\right), \ldots, y\left(h_{k}(i)\right)\right)
$$$$
+\frac{1}{T b_{*}} \sum_{i=T_{1}+1}^{\infty} \mid h\left(i, y^{w}\left(h_{1}(i)\right), \ldots, y^{w}\left(h_{k}(i)\right)\right)
$$

$$
\begin{array}{r}
-h\left(i, y\left(h_{1}(i)\right), \ldots, y\left(h_{k}(i)\right)\right) \mid \\
+\frac{1}{T b_{*}} \sum_{i=T+\tau}^{T_{1}} \sum_{s=i}^{T_{2}} \mid g\left(s, y^{w}\left(g_{1}(s)\right), \ldots, y^{w}\left(g_{k}(s)\right)\right) \\
-g\left(s, y\left(g_{1}(s)\right), \ldots, y\left(g_{k}(s)\right)\right) \mid
\end{array}
$$$$
+\frac{1}{T b_{*}} \sum_{i=T+\tau}^{T_{1}} \sum_{s=T_{2}+1}^{\infty} \mid g\left(s, y^{w}\left(g_{1}(s)\right), \ldots, y^{w}\left(g_{k}(s)\right)\right)
$$$$
-g\left(s, y\left(g_{1}(s)\right), \ldots, y\left(g_{k}(s)\right)\right)
$$$$
+\frac{1}{T b_{*}} \sum_{i=T_{1}+1}^{\infty} \sum_{s=i}^{\infty} \mid g\left(s, y^{w}\left(g_{1}(s)\right), \ldots, y^{w}\left(g_{k}(s)\right)\right)
$$$$
-g\left(s, y\left(g_{1}(s)\right), \ldots, y\left(g_{k}(s)\right)\right)
$$$$
+\frac{1}{T b_{*}} \sum_{i=T+\tau}^{T_{1}} \sum_{s=i}^{T_{2}} \sum_{t=s}^{T_{3}} \mid f\left(t, y^{w}\left(f_{1}(t)\right), \ldots, y^{w}\left(f_{k}(t)\right)\right)
$$$$
-f\left(t, y\left(f_{1}(t)\right), \ldots, y\left(f_{k}(t)\right)\right) \mid
$$$$
+\frac{1}{T b_{*}} \sum_{i=T+\tau}^{T_{1}} \sum_{s=i}^{T_{2}} \sum_{t=T_{3}+1}^{\infty} \mid f\left(t, y^{w}\left(f_{1}(t)\right), \ldots, y^{w}\left(f_{k}(t)\right)\right)
$$$$
-f\left(t, y\left(f_{1}(t)\right), \ldots, y\left(f_{k}(t)\right)\right)
$$$$
+\frac{1}{T b_{*}} \sum_{i=T+\tau}^{T_{1}} \sum_{s=T_{2}+1}^{\infty} \sum_{t=s}^{\infty} \mid f\left(t, y^{w}\left(f_{1}(t)\right), \ldots, y^{w}\left(f_{k}(t)\right)\right)
$$$$
-f\left(t, y\left(f_{1}(t)\right), \ldots, y\left(f_{k}(t)\right)\right)
$$ 


$$
\begin{aligned}
& +\frac{1}{T b_{*}} \sum_{i=T_{1}+1}^{\infty} \sum_{s=i}^{\infty} \sum_{t=s}^{\infty} \mid f\left(t, y^{w}\left(f_{1}(t)\right), \ldots, y^{w}\left(f_{k}(t)\right)\right) \\
& <\frac{\varepsilon}{16}+\frac{2}{T b_{*}} \sum_{i=T_{1}+1}^{\infty} H_{i}+\frac{\varepsilon}{16} \\
& +\frac{2}{T b_{*}} \sum_{i=T+\tau}^{T_{1}} \sum_{s=T_{2}+1}^{\infty} G_{s}+\frac{2}{T b_{*}} \sum_{i=T_{1}+1}^{\infty} \sum_{s=i}^{\infty} G_{s} \\
& +\frac{\varepsilon}{16}+\frac{2}{T b_{*}} \sum_{i=T+\tau}^{T_{1}} \sum_{s=i}^{T_{2}} \sum_{t=T_{3}+1}^{\infty} F_{t} \\
& +\frac{2}{T b_{*}} \sum_{i=T+\tau}^{T_{1}} \sum_{s=T_{2}+1}^{\infty} \sum_{t=s}^{\infty} F_{t}+\frac{2}{T b_{*}} \sum_{i=T_{1}+1}^{\infty} \sum_{s=i}^{\infty} \sum_{t=s}^{\infty} F_{t} \\
& \left.\left.<\varepsilon, \quad w \geq T_{4}, f_{k}(t)\right)\right) \mid
\end{aligned}
$$

which implies that $G_{L}$ is continuous in $\Omega\left(A_{*}, B^{*}, T\right)$.

It follows from (47) and (51) that for any $x=\{x(n)\}_{n \in \mathbb{N}_{\beta}} \in$ $\Omega\left(A_{*}, B^{*}, T\right)$ and $t_{1}, t_{2} \geq T_{4}$

$$
\begin{aligned}
& \left|\frac{\left(G_{L} y\right)\left(t_{2}\right)}{t_{2}}-\frac{\left(G_{L} y\right)\left(t_{1}\right)}{t_{1}}\right| \\
& =\mid \frac{1}{t_{2} b(n+\tau)} \\
& \sum_{i=t_{2}+\tau}^{\infty} h\left(i, y\left(h_{1}(i)\right), \ldots, y\left(h_{k}(i)\right)\right) \\
& -\frac{1}{t_{1} b(n+\tau)} \\
& \cdot \sum_{i=t_{1}+\tau}^{\infty} h\left(i, y\left(h_{1}(i)\right), \ldots, y\left(h_{k}(i)\right)\right) \\
& -\frac{1}{t_{2} b(n+\tau)} \\
& \cdot \sum_{i=t_{2}+\tau}^{\infty} \sum_{s=i}^{\infty} g\left(s, y\left(g_{1}(s)\right), \ldots, y\left(g_{k}(s)\right)\right) \\
& +\frac{1}{t_{1} b(n+\tau)} \\
& \cdot \sum_{i=t_{1}+\tau}^{\infty} \sum_{s=i}^{\infty} g\left(s, y\left(g_{1}(s)\right), \ldots, y\left(g_{k}(s)\right)\right) \\
& +\frac{1}{t_{2} b(n+\tau)}
\end{aligned}
$$

which means that $G_{L}\left(\Omega\left(A_{*}, B, T\right)\right)$ is uniformly Cauchy, which together with (49) and Lemma 1 yields that $G_{L}\left(\Omega\left(A_{*}\right.\right.$, $\left.\left.B^{*}, T\right)\right)$ is relatively compact. That is, $G_{L}$ is completely continuous in $\Omega\left(A_{*}, B^{*}, T\right)$. Thus (44), (48), and Lemma 2 ensure that the mapping $S_{L}+G_{L}$ has a fixed point $x=\{x(n)\}_{n \in \mathbb{N}_{\beta}} \in$ $\Omega\left(A_{*}, B^{*}, T\right)$, which together with (46) and (47) yields that

$$
\begin{aligned}
x(n)= & \frac{n L}{b(n+\tau)}-\frac{x(n+\tau)}{b(n+\tau)}-\frac{c(n+\tau)}{b(n+\tau)} \\
& +\frac{1}{b(n+\tau)} \sum_{i=n+\tau}^{\infty} h\left(i, x\left(h_{1}(i)\right), \ldots, x\left(h_{k}(i)\right)\right) \\
& -\frac{1}{b(n+\tau)} \sum_{i=n+\tau}^{\infty} \sum_{s=i}^{\infty} g\left(s, x\left(g_{1}(s)\right), \ldots, x\left(g_{k}(s)\right)\right) \\
& +\frac{1}{b(n+\tau)} \\
& \cdot \sum_{i=n+\tau}^{\infty} \sum_{s=i}^{\infty} \sum_{t=s}^{\infty}\left[f\left(t, x\left(f_{1}(t)\right), \ldots, x\left(f_{k}(t)\right)\right)-d(t)\right], \quad n \geq T,
\end{aligned}
$$

which means that

$$
\begin{aligned}
x(n+\tau) & +b(n+\tau) x(n)+c(n+\tau) \\
= & n L+\sum_{i=n+\tau}^{\infty} h\left(i, x\left(h_{1}(i)\right), \ldots, x\left(h_{k}(i)\right)\right) \\
& -\sum_{i=n+\tau}^{\infty} \sum_{s=i}^{\infty} g\left(s, x\left(g_{1}(s)\right), \ldots, x\left(g_{k}(s)\right)\right) \\
& +\sum_{i=n+\tau}^{\infty} \sum_{s=i}^{\infty} \sum_{t=s}^{\infty}\left[f\left(t, x\left(f_{1}(t)\right), \ldots, x\left(f_{k}(t)\right)\right)-d(t)\right],
\end{aligned}
$$


It follows from (56) that

$$
\begin{aligned}
\Delta(x(n)+b(n) x(n-\tau)+c(n)) \\
=L-h\left(n, x\left(h_{1}(n)\right), \ldots, x\left(h_{k}(n)\right)\right) \\
\quad+\sum_{s=n}^{\infty} g\left(s, x\left(g_{1}(s)\right), x\left(g_{2}(s)\right), \ldots, x\left(g_{k}(s)\right)\right) \\
\quad-\sum_{s=n}^{\infty} \sum_{t=s}^{\infty}\left[f\left(t, x\left(f_{1}(t)\right), \ldots, x\left(f_{k}(t)\right)\right)-d(t)\right],
\end{aligned}
$$$$
n \geq T+\tau
$$$$
\Delta^{3}(x(n)+b(n) x(n-\tau)+c(n))
$$$$
=-\Delta^{2} h\left(n, x\left(h_{1}(n)\right), \ldots, x\left(h_{k}(n)\right)\right)
$$$$
-\Delta g\left(n, x\left(g_{1}(n)\right), \ldots, x\left(g_{k}(n)\right)\right)
$$$$
-\left[f\left(n, x\left(f_{1}(n)\right), \ldots, x\left(f_{k}(n)\right)\right)-d(n)\right]
$$$$
n \geq T+\tau,
$$

that is, $x=\{x(n)\}_{n \in \mathbb{N}_{\beta}} \in \Omega\left(A_{*}, B^{*}, T\right)$ is a positive solution of (4). By means of (15)-(17) and (56), we deduce that

$$
\begin{aligned}
& \left|\frac{x(n)+b(n) x(n-\tau)+c(n)}{n}-L\right| \\
& =\mid-\frac{\tau}{n}+\frac{1}{n} \sum_{i=n}^{\infty} h\left(i, x\left(h_{1}(i)\right), \ldots, x\left(h_{k}(i)\right)\right) \\
& \quad-\frac{1}{n} \sum_{i=n}^{\infty} \sum_{s=i}^{\infty} g\left(s, x\left(g_{1}(s)\right), \ldots, x\left(g_{k}(s)\right)\right) \\
& \quad+\frac{1}{n} \sum_{i=n}^{\infty} \sum_{s=i}^{\infty} \sum_{t=s}^{\infty}\left[f\left(t, x\left(f_{1}(t)\right), \ldots, x\left(f_{k}(t)\right)\right)\right. \\
& \leq \frac{\tau}{n}+\frac{1}{n} \sum_{i=n}^{\infty} H_{i}+\frac{1}{n} \sum_{i=n}^{\infty} \sum_{s=i}^{\infty} G_{s} \\
& +\frac{1}{n} \sum_{i=n}^{\infty} \sum_{s=i}^{\infty} \sum_{t=s}^{\infty} F_{t} \longrightarrow 0 \text { as } n \longrightarrow \infty, \\
& \quad-d(t)] \mid
\end{aligned}
$$

which ensures that (41) holds. The proof of (19) is similar to that of Theorem 5 and is omitted. (ii) Let $L_{1}, L_{2} \in\left(b^{*} A+\left(B^{*}+c^{*}\right)\left(b^{*} / b_{*}\right), b_{*} B+b_{*} A_{*} / b^{*}-\right.$ $\left.c^{*}\right)$ and $L_{1} \neq L_{2}$. As in the proof of (i), we deduce that, for each $l \in\{1,2\}$, there exist $\theta_{l} \in(0,1), T_{l} \geq n_{0}+\tau+\alpha$, and two mappings $S_{L_{l}}$ and $G_{L_{l}}: \Omega\left(A_{*}, B^{*}, T_{l}\right) \rightarrow l_{\beta}^{\infty}$ satisfying (43)-(47), where $\theta, T, L, S_{L}$, and $G_{L}$ are replaced by $\theta_{l}, T_{l}, L_{l}$, $S_{L_{l}}$, and $G_{L_{l}}$, respectively, and $S_{L_{l}}+G_{L_{l}}$ possesses a fixed point $x_{l}=\left\{x_{l}(n)\right\}_{n \in \mathbb{N}_{\beta}} \in \Omega\left(A_{*}, B^{*}, T_{l}\right)$, which is a positive solution of (4); that is,

$$
\begin{aligned}
& x_{l}(n)= \frac{n L}{b(n+\tau)}-\frac{x_{l}(n+\tau)}{b(n+\tau)}-\frac{c(n+\tau)}{b(n+\tau)}+\frac{1}{b(n+\tau)} \\
& \cdot \sum_{i=n+\tau}^{\infty} h\left(i, x_{l}\left(h_{1}(i)\right), \ldots, x_{l}\left(h_{k}(i)\right)\right)-\frac{1}{b(n+\tau)} \\
& \cdot \sum_{i=n+\tau}^{\infty} \sum_{s=i}^{\infty} g\left(s, x_{l}\left(g_{1}(s)\right), \ldots, x_{l}\left(g_{k}(s)\right)\right)+\frac{1}{b(n+\tau)} \\
& \cdot \sum_{i=n+\tau}^{\infty} \sum_{s=i}^{\infty} \sum_{t=s}^{\infty}\left[f\left(t, x_{l}\left(f_{1}(t)\right), \ldots, x_{l}\left(f_{k}(t)\right)\right)\right. \\
&-d(t)], \quad n \geq T_{l} .
\end{aligned}
$$

Note that (16) and (17) imply that there exists $T_{*}>\max \left\{T_{1}\right.$, $T_{2}$ \} satisfying

$$
\begin{aligned}
\frac{1}{T_{*} b_{*}} & \left(\sum_{i=T_{*}+\tau}^{\infty} H_{i}+\sum_{i=T_{*}+\tau}^{\infty} \sum_{s=i}^{\infty} G_{s}+\sum_{i=T_{*}+\tau}^{\infty} \sum_{s=i}^{\infty} \sum_{t=s}^{\infty} F_{t}\right) \\
& <\frac{\left|L_{1}-L_{2}\right|}{4 b^{*}} .
\end{aligned}
$$

In view of (15), (59), and (60), we infer that for any $n \geq T_{*}$

$$
\begin{aligned}
& \left|\frac{x_{1}(n)}{n}-\frac{x_{2}(n)}{n}\right| \\
& =\mid \frac{L_{1}-L_{2}}{b(n+\tau)}-\frac{x_{1}(n+\tau)-x_{2}(n+\tau)}{n b(n+\tau)}+\frac{1}{n b(n+\tau)} \\
& \quad \sum_{i=n+\tau}^{\infty}\left[h\left(i, x_{1}\left(h_{1}(i)\right), \ldots, x_{1}\left(h_{k}(i)\right)\right)\right. \\
& -\frac{1}{n b(n+\tau)}
\end{aligned}
$$




$$
\begin{aligned}
& \cdot \sum_{i=n+\tau}^{\infty} \sum_{s=i}^{\infty}\left[g\left(s, x_{1}\left(g_{1}(s)\right), \ldots, x_{1}\left(g_{k}(s)\right)\right)\right. \\
& \left.-g\left(s, x_{2}\left(g_{1}(s)\right), \ldots, x_{2}\left(g_{k}(s)\right)\right)\right] \\
& +\frac{1}{n b(n+\tau)} \\
& \cdot \sum_{i=n+\tau}^{\infty} \sum_{s=i}^{\infty} \sum_{t=s}^{\infty}\left[f\left(t, x_{1}\left(f_{1}(t)\right), \ldots, x_{1}\left(f_{k}(t)\right)\right)\right. \\
& \left.-f\left(t, x_{2}\left(f_{1}(t)\right), \ldots, x_{2}\left(f_{k}(t)\right)\right)\right] \\
& \geq \frac{\left|L_{1}-L_{2}\right|}{b^{*}}-\frac{n+\tau}{n b(n+\tau)}\left|\frac{x_{1}(n+\tau)-x_{2}(n+\tau)}{n+\tau}\right| \\
& -\frac{1}{T_{*} b_{*}} \sum_{i=T_{*}+\tau}^{\infty} \mid h\left(i, x_{1}\left(h_{1}(i)\right), \ldots, x_{1}\left(h_{k}(i)\right)\right) \\
& -h\left(i, x_{2}\left(h_{1}(i)\right), \ldots, x_{2}\left(h_{k}(i)\right)\right) \\
& -\frac{1}{T_{*} b_{*}} \sum_{i=T_{*}+\tau}^{\infty} \sum_{s=i}^{\infty} \mid g\left(s, x_{1}\left(g_{1}(s)\right), \ldots, x_{1}\left(g_{k}(s)\right)\right) \\
& -g\left(s, x_{2}\left(g_{1}(s)\right), \ldots, x_{2}\left(g_{k}(s)\right)\right) \mid \\
& -\frac{1}{T_{*} b_{*}} \sum_{i=T_{*}+\tau}^{\infty} \sum_{s=i}^{\infty} \sum_{t=s}^{\infty} \mid f\left(t, x_{1}\left(f_{1}(t)\right), \ldots, x_{1}\left(f_{k}(t)\right)\right) \\
& -f\left(t, x_{2}\left(f_{1}(t)\right), \ldots, x_{2}\left(f_{k}(t)\right)\right) \\
& \geq \frac{\left|L_{1}-L_{2}\right|}{b^{*}}-\frac{1}{b_{*}}\left(1+\frac{\tau}{T_{*}}\right)\left\|x_{1}-x_{2}\right\| \\
& -\frac{2}{T_{*} b_{*}} \\
& \cdot\left(\sum_{i=T_{*}+\tau}^{\infty} H_{i}+\sum_{i=T_{*}+\tau}^{\infty} \sum_{s=i}^{\infty} G_{s}+\sum_{i=T_{*}+\tau}^{\infty} \sum_{s=i}^{\infty} \sum_{t=s}^{\infty} F_{t}\right) \\
& >\frac{\left|L_{1}-L_{2}\right|}{2 b^{*}}-\frac{1}{b_{*}}\left(1+\frac{\tau}{T_{*}}\right)\left\|x_{1}-x_{2}\right\|,
\end{aligned}
$$

which implies that

$$
\left\|x_{1}-x_{2}\right\|>\frac{\left|L_{1}-L_{2}\right|}{2 b^{*}\left(1+\left(1 / b_{*}\right)\left(1+\tau / T_{*}\right)\right)}>0,
$$

which yields that $x_{1} \neq x_{2}$. That is, (4) possesses uncountably many positive solutions in $l_{\beta}^{\infty}$. This completes the proof.
Theorem 7. Assume that there exist constants $b_{*}$ and $b^{*}$ and three nonnegative sequences $\left\{F_{n}\right\}_{n \in \mathbb{N}_{n_{0}}},\left\{G_{n}\right\}_{n \in \mathbb{N}_{n_{0}}}$, and $\left\{H_{n}\right\}_{n \in \mathbb{N}_{n_{0}}}$ satisfying (15)-(17) and

$$
A<B+b_{*} B^{*}, \quad-1<b_{*} \leq b(n) \leq b^{*} \leq 0, n \in \mathbb{N}_{n_{0}} .
$$

Then

(i) equation (4) possesses a positive solution $x=$ $\{x(n)\}_{n \in \mathbb{N}_{\beta}} \in l_{\beta}^{\infty}$ with (19) and

$$
\lim _{n \rightarrow \infty} \frac{x(n)+b(n) x(n-\tau)+c(n)}{n} \in\left(A, B+b_{*} B^{*}\right) ;
$$

(ii) equation (4) possesses uncountably many positive solutions in $l_{\beta}^{\infty}$.

Proof. (i) Let $L \in\left(A, B+b_{*} B^{*}\right)$. It follows from (16) and (17) that there exists $T \geq n_{0}+\tau+\alpha$ satisfying

$$
\begin{gathered}
\frac{1}{T} \sum_{i=T}^{\infty}\left\{H_{i}+\sum_{s=i}^{\infty} G_{s}+\sum_{s=i}^{\infty} \sum_{t=s}^{\infty}\left[F_{t}+|d(t)|\right]\right\} \\
<\min \left\{L-A, B+b_{*} B^{*}-L\right\} .
\end{gathered}
$$

Define two mappings $S_{L}$ and $G_{L}: \Omega\left(A_{*}, B^{*}, T\right) \rightarrow l_{\beta}^{\infty}$ by (21) and (22).

Now we show that (23), (66) below hold:

$$
\left\|S_{L} x-S_{L} y\right\| \leq\left|b_{*}\right|\|x-y\|, \quad x, y \in \Omega\left(A_{*}, B^{*}, T\right) ;
$$$$
\left\|G_{L} y\right\| \leq B, \quad y \in \Omega\left(A_{*}, B^{*}, T\right) .
$$

Using (15), (21), (22), (63), and (65), we get that for any $x=$ $\{x(n)\}_{n \in \mathbb{N}_{\beta}}, y=\{y(n)\}_{n \in \mathbb{N}_{\beta}} \in \Omega\left(A_{*}, B^{*}, T\right)$,

$$
\begin{aligned}
& \frac{\left(S_{L} x\right)(n)+\left(G_{L} y\right)(n)}{n} \\
& =L-\frac{b(n)}{n} x(n-\tau)-\frac{c(n)}{n} \\
& \quad+\frac{1}{n} \sum_{i=n}^{\infty} h\left(i, y\left(h_{1}(i)\right), \ldots, y\left(h_{k}(i)\right)\right)
\end{aligned}
$$

$$
\begin{aligned}
& -\frac{1}{n} \sum_{i=n}^{\infty} \sum_{s=i}^{\infty} g\left(s, y\left(g_{1}(s)\right), \ldots, y\left(g_{k}(s)\right)\right) \\
& +\frac{1}{n} \sum_{i=n}^{\infty} \sum_{s=i}^{\infty} \sum_{t=s}^{\infty}\left[f\left(t, y\left(f_{1}(t)\right), \ldots, y\left(f_{k}(t)\right)\right)-d(t)\right]
\end{aligned}
$$




$$
\begin{aligned}
& \leq L-\frac{(n-\tau) b(n)}{n} \cdot \frac{x(n-\tau)}{n-\tau}+\frac{|c(n)|}{n} \\
& \geq L-\frac{|c(n)|}{n}-\frac{1}{T} \sum_{i=T}^{\infty} H_{i}-\frac{1}{T} \sum_{i=T}^{\infty} \sum_{s=i}^{\infty} G_{s} \\
& +\frac{1}{n} \sum_{i=n}^{\infty}\left|h\left(i, y\left(h_{1}(i)\right), \ldots, y\left(h_{k}(i)\right)\right)\right| \\
& +\frac{1}{n} \sum_{i=n}^{\infty} \sum_{s=i}^{\infty}\left|g\left(s, y\left(g_{1}(s)\right), \ldots, y\left(g_{k}(s)\right)\right)\right| \\
& +\frac{1}{n} \sum_{i=n}^{\infty} \sum_{s=i}^{\infty} \sum_{t=s}^{\infty}\left[\left|f\left(t, y\left(f_{1}(t)\right), \ldots, y\left(f_{k}(t)\right)\right)\right|+|d(t)|\right] \\
& \leq L-b_{*} B^{*}+\frac{|c(n)|}{n}+\frac{1}{T} \sum_{i=T}^{\infty} H_{i}+\frac{1}{T} \sum_{i=T}^{\infty} \sum_{s=i}^{\infty} G_{s} \\
& +\frac{1}{T} \sum_{i=T}^{\infty} \sum_{s=i}^{\infty} \sum_{t=s}^{\infty}\left[F_{t}+|d(t)|\right]<L-b_{*} B^{*} \\
& +\frac{|c(n)|}{n}+\min \left\{L-A, B+b_{*} B^{*}-L\right\} \leq B+\frac{|c(n)|}{n} \\
& =B(n), \quad n \geq T, \\
& \frac{\left(S_{L} x\right)(n)+\left(G_{L} y\right)(n)}{n}=\frac{n}{T} \cdot \frac{\left(S_{L} x\right)(T)+\left(G_{L} y\right)(T)}{n} \\
& \leq B(T), \quad \beta \leq n<T, \\
& \frac{\left(S_{L} x\right)(n)+\left(G_{L} y\right)(n)}{n} \\
& =L-\frac{b(n)}{n} x(n-\tau)-\frac{c(n)}{n} \\
& +\frac{1}{n} \sum_{i=n}^{\infty} h\left(i, y\left(h_{1}(i)\right), \ldots, y\left(h_{k}(i)\right)\right) \\
& -\frac{1}{n} \sum_{i=n s=i}^{\infty} \sum^{\infty} g\left(s, y\left(g_{1}(s)\right), \ldots, y\left(g_{k}(s)\right)\right) \\
& +\frac{1}{n} \sum_{i=n s=i}^{\infty} \sum_{i=s}^{\infty} \sum_{t=s}^{\infty}\left[f\left(t, y\left(f_{1}(t)\right), \ldots, y\left(f_{k}(t)\right)\right)-d(t)\right] \\
& \geq L-\frac{|c(n)|}{n}-\frac{1}{n} \sum_{i=n}^{\infty}\left|h\left(i, y\left(h_{1}(i)\right), \ldots, y\left(h_{k}(i)\right)\right)\right| \\
& -\frac{1}{n} \sum_{i=n s=i}^{\infty} \sum^{\infty}\left|g\left(s, y\left(g_{1}(s)\right), \ldots, y\left(g_{k}(s)\right)\right)\right| \\
& -\frac{1}{n} \sum_{i=n s=i}^{\infty} \sum_{i=s}^{\infty} \sum_{t=s}^{\infty}\left[f\left(t, y\left(f_{1}(t)\right), \ldots, y\left(f_{k}(t)\right)\right)|+| d(t) \mid\right] \\
& \begin{array}{r}
-\frac{1}{T} \sum_{i=T}^{\infty} \sum_{s=i}^{\infty} \sum_{t=s}^{\infty}\left[F_{t}+|d(t)|\right]>L-\frac{|c(n)|}{n} \\
-\min \left\{L-A, B+b_{*} B^{*}-L\right\} \geq A-\frac{|c(n)|}{n}=A(n), \\
n \geq T,
\end{array} \\
& \frac{\left(S_{L} x\right)(n)+\left(G_{L} y\right)(n)}{n}=\frac{n}{T} \cdot \frac{\left(S_{L} x\right)(T)+\left(G_{L} y\right)(T)}{n} \\
& \geq A(T), \quad \beta \leq n<T, \\
& \left|\frac{\left(S_{L} x\right)(n)-\left(S_{L} y\right)(n)}{n}\right|=|b(n)| \frac{n-\tau}{n}\left|\frac{x(n-\tau)-y(n-\tau)}{n-\tau}\right| \\
& \leq\left|b_{*}\right|\|x-y\|, \quad n \geq T, \\
& \left|\frac{\left(S_{L} x\right)(n)-\left(S_{L} y\right)(n)}{n}\right|=\frac{n}{T}\left|\frac{\left(S_{L} x\right)(T)-\left(S_{L} y\right)(T)}{n}\right| \\
& \leq\left|b_{*}\right|\|x-y\|, \quad \beta \leq n<T, \\
& \left|\frac{\left(G_{L} y\right)(n)}{n}\right| \\
& =\mid \frac{1}{n} \sum_{i=n}^{\infty} h\left(i, y\left(h_{1}(i)\right), \ldots, y\left(h_{k}(i)\right)\right) \\
& -\frac{1}{n} \sum_{i=n s=i}^{\infty} \sum_{s=i}^{\infty} g\left(s, y\left(g_{1}(s)\right), \ldots, y\left(g_{k}(s)\right)\right) \\
& +\frac{1}{n} \sum_{i=n s=i}^{\infty} \sum^{\infty} \sum_{t=s}^{\infty}\left[f\left(t, y\left(f_{1}(t)\right), \ldots, y\left(f_{k}(t)\right)\right)-d(t)\right] \mid \\
& \leq \frac{1}{n} \sum_{i=n}^{\infty}\left|h\left(i, y\left(h_{1}(i)\right), \ldots, y\left(h_{k}(i)\right)\right)\right| \\
& +\frac{1}{n} \sum_{i=n s=i}^{\infty} \sum_{s}^{\infty}\left|g\left(s, y\left(g_{1}(s)\right), \ldots, y\left(g_{k}(s)\right)\right)\right| \\
& +\frac{1}{n} \sum_{i=n s=i}^{\infty} \sum_{i=s}^{\infty} \sum^{\infty}\left[\left|f\left(t, y\left(f_{1}(t)\right), \ldots, y\left(f_{k}(t)\right)\right)\right|+|d(t)|\right] \\
& \leq \frac{1}{T} \sum_{i=T}^{\infty} H_{i}+\frac{1}{T} \sum_{i=T}^{\infty} \sum_{s=i}^{\infty} G_{s} \\
& +\frac{1}{T} \sum_{i=T}^{\infty} \sum_{s=i}^{\infty} \sum_{t=s}^{\infty}\left[F_{t}+|d(t)|\right]
\end{aligned}
$$




$$
\begin{gathered}
<\min \left\{L-A, B+b_{*} B^{*}-L\right\} \leq B, \quad n \geq T, \\
\left|\frac{\left(G_{L} y\right)(n)}{n}\right|=\frac{n}{T}\left|\frac{\left(G_{L} y\right)(T)}{n}\right| \leq B, \quad \beta \leq n<T,
\end{gathered}
$$

which yield (21) and (66). The rest of the proof is similar to that of Theorem 5. This completes the proof.

Theorem 8. Assume that there exist constants $b_{*}$ and $b^{*}$ and three nonnegative sequences $\left\{F_{n}\right\}_{n \in \mathbb{N}_{n_{0}}},\left\{G_{n}\right\}_{n \in \mathbb{N}_{n_{0}}}$, and $\left\{H_{n}\right\}_{n \in \mathbb{N}_{n_{0}}}$ satisfying (15)-(17) and

$$
\begin{array}{r}
b^{*} B+B^{*}+c^{*}<b_{*} A+A_{*}-\frac{b_{*} c^{*}}{b^{*}}<0, \\
b_{*} \leq b(n) \leq b^{*}<-1, \quad n \in \mathbb{N}_{n_{0}} .
\end{array}
$$

Then

(i) equation (4) possesses a positive solution $x=$ $\{x(n)\}_{n \in \mathbb{N}_{\beta}} \in l_{\beta}^{\infty}$ with (19) and

$$
\begin{aligned}
& \lim _{n \rightarrow \infty} \frac{x(n)+b(n) x(n-\tau)+c(n)}{n} \\
& \quad \in\left(b^{*} B+B^{*}+c^{*}, b_{*} A+A_{*}-\frac{b_{*} c^{*}}{b^{*}}\right) ;
\end{aligned}
$$

(ii) equation (4) possesses uncountably many positive solutions in $l_{\beta}^{\infty}$.

Proof. (i) Let $L \in\left(b^{*} B+\left(B^{*}+c^{*}\right), b_{*} A+A_{*}-b_{*} c^{*} / b^{*}\right)$. Notice that

$$
\begin{aligned}
\lim _{n \rightarrow \infty} & {\left[b^{*} B+\left(B^{*}+c^{*}\right)\left(1+\frac{\tau}{n}\right)\right] } \\
& =b^{*} B+B^{*}+c^{*}<L<b_{*} A+A_{*}-\frac{b_{*} c^{*}}{b^{*}} \\
& =\lim _{n \rightarrow \infty}\left[b_{*} A+A_{*}-\frac{b_{*} c^{*}}{b^{*}}\left(1+\frac{\tau}{n}\right)\right],
\end{aligned}
$$

which means that there exists $N \in \mathbb{N}$ satisfying

$$
\begin{aligned}
b^{*} B+\left(B^{*}+c^{*}\right) & <b^{*} B+\left(B^{*}+c^{*}\right)\left(1+\frac{\tau}{N}\right) \\
& <L<b_{*} A+A_{*}-\frac{b_{*} c^{*}}{b^{*}}\left(1+\frac{\tau}{N}\right) \\
& <b_{*} A+A_{*}-\frac{b_{*} c^{*}}{b^{*}} .
\end{aligned}
$$

It follows from (16) and (17) that there exist $\theta \in(0,1)$ and $T>\max \left\{N, n_{0}+\tau+\alpha\right\}$ satisfying

$$
\theta=\frac{1}{\left|b^{*}\right|}\left(1+\frac{\tau}{T}\right)
$$

$$
\begin{gathered}
\frac{1}{T} \sum_{i=T+\tau}^{\infty}\left\{H_{i}+\sum_{s=i}^{\infty} G_{s}+\sum_{s=i}^{\infty} \sum_{t=s}^{\infty}\left[F_{t}+|d(t)|\right]\right\} \\
<\min \left\{L-b^{*} B-\left(1+\frac{\tau}{N}\right)\left(B^{*}+c^{*}\right),\right. \\
\left.b^{*} A+\frac{b^{*} A_{*}}{b_{*}}-c^{*}\left(1+\frac{\tau}{N}\right)-\frac{b^{*}}{b_{*}} L\right\} \\
<\min \left\{L-b^{*} B-\left(1+\frac{\tau}{T}\right)\left(B^{*}+c^{*}\right),\right. \\
\left.b^{*} A+\frac{b^{*} A_{*}}{b_{*}}-c^{*}\left(1+\frac{\tau}{T}\right)-\frac{b^{*}}{b_{*}} L\right\} .
\end{gathered}
$$

Define two mappings $S_{L}$ and $G_{L}: \Omega\left(A_{*}, B^{*}, T\right) \rightarrow l_{\beta}^{\infty}$ by (46) and (47).

Now we show that (23), (25), and (48) hold. Using (15), (46), (47), (68), and (72), we get that for any $x=\{x(n)\}_{n \in \mathbb{N}_{\beta}}$, $y=\{y(n)\}_{n \in \mathbb{N}_{\beta}} \in \Omega\left(A_{*}, B^{*}, T\right)$

$$
\begin{aligned}
& \frac{\left(S_{L} x\right)(n)+\left(G_{L} y\right)(n)}{n} \\
= & \frac{L}{b(n+\tau)}-\frac{x(n+\tau)}{n b(n+\tau)}-\frac{c(n+\tau)}{n b(n+\tau)}+\frac{1}{n b(n+\tau)} \\
& \cdot \sum_{i=n+\tau}^{\infty} h\left(i, y\left(h_{1}(i)\right), \ldots, y\left(h_{k}(i)\right)\right)-\frac{1}{n b(n+\tau)} \\
& \cdot \sum_{i=n+\tau}^{\infty} \sum_{s=i}^{\infty} g\left(s, y\left(g_{1}(s)\right), \ldots, y\left(g_{k}(s)\right)\right)+\frac{1}{n b(n+\tau)} \\
& \cdot \sum_{i=n+\tau}^{\infty} \sum_{s=i}^{\infty} \sum_{t=s}^{\infty}\left[f\left(t, y\left(f_{1}(t)\right), \ldots, y\left(f_{k}(t)\right)\right)-d(t)\right] \\
& \frac{L}{b^{*}}-\frac{\sum_{i=n+\tau}}{n b(n+\tau)} \cdot \frac{x(n+\tau)}{(n+\tau)}-\frac{n+\tau}{n b(n+\tau)} \\
& \cdot \sum_{i=n+\tau}^{\infty} \sum_{s=i}^{\infty}\left|g\left(s, y\left(g_{1}(s)\right), \ldots, y\left(g_{k}(s)\right)\right)\right| \\
& \cdot \frac{|c(n+\tau)|}{n+\tau}-\frac{1}{n b(n+\tau)} \\
& \sum_{i=n+\tau}^{\infty}\left|h\left(i, y\left(h_{1}(i)\right), \ldots, y\left(h_{k}(i)\right)\right)\right|-\frac{1}{n b(n+\tau)}
\end{aligned}
$$




$$
\begin{aligned}
& \leq \frac{L}{b^{*}}-\frac{1}{b^{*}}\left(1+\frac{\tau}{T}\right) B^{*}+\frac{c^{*}}{b^{*}}\left(1+\frac{\tau}{T}\right) \\
& \cdot \sum_{i=n+\tau}^{\infty} \sum_{s=i}^{\infty}\left|g\left(s, y\left(g_{1}(s)\right), \ldots, y\left(g_{k}(s)\right)\right)\right| \\
& -\frac{1}{T b^{*}} \sum_{i=T+\tau}^{\infty} H_{i}-\frac{1}{T b^{*}} \sum_{i=T+\tau}^{\infty} \sum_{s=i}^{\infty} G_{s} \\
& -\frac{1}{T b^{*}} \sum_{i=T+\tau}^{\infty} \sum_{s=i}^{\infty} \sum_{t=s}^{\infty}\left[F_{t}+|d(t)|\right] \\
& <\frac{L}{b^{*}}-\frac{1}{b^{*}}\left(1+\frac{\tau}{T}\right)\left(B^{*}+c^{*}\right) \\
& -\frac{1}{b^{*}} \min \left\{L-b^{*} B-\left(1+\frac{\tau}{T}\right)\left(B^{*}+c^{*}\right),\right. \\
& \left.b^{*} A+\frac{b^{*} A_{*}}{b_{*}}-c^{*}\left(1+\frac{\tau}{T}\right)-\frac{b^{*}}{b_{*}} L\right\} \\
& \leq B \leq B(n), \quad n \geq T, \\
& \frac{\left(S_{L} x\right)(n)+\left(G_{L} y\right)(n)}{n}=\frac{n}{T} \cdot \frac{\left(S_{L} x\right)(T)+\left(G_{L} y\right)(T)}{n} \\
& \leq B(T), \quad \beta \leq n<T, \\
& \frac{\left(S_{L} x\right)(n)+\left(G_{L} y\right)(n)}{n} \\
& =\frac{L}{b(n+\tau)}-\frac{x(n+\tau)}{n b(n+\tau)}-\frac{c(n+\tau)}{n b(n+\tau)}+\frac{1}{n b(n+\tau)} \\
& \cdot \sum_{i=n+\tau}^{\infty} h\left(i, y\left(h_{1}(i)\right), \ldots, y\left(h_{k}(i)\right)\right) \\
& -\frac{1}{n b(n+\tau)} \\
& \cdot \sum_{i=n+\tau}^{\infty} \sum_{s=i}^{\infty} g\left(s, y\left(g_{1}(s)\right), \ldots, y\left(g_{k}(s)\right)\right) \\
& +\frac{1}{n b(n+\tau)} \\
& \cdot \sum_{i=n+\tau}^{\infty} \sum_{s=i}^{\infty} \sum_{t=s}^{\infty}\left[f\left(t, y\left(f_{1}(t)\right), \ldots, y\left(f_{k}(t)\right)\right)-d(t)\right] \\
& \geq \frac{L}{b_{*}}-\frac{n+\tau}{n b(n+\tau)} \cdot \frac{x(n+\tau)}{n+\tau}+\frac{n+\tau}{n b(n+\tau)} \cdot \frac{|c(n+\tau)|}{n+\tau} \\
& +\frac{1}{n b(n+\tau)} \sum_{i=n+\tau}^{\infty}\left|h\left(i, y\left(h_{1}(i)\right), \ldots, y\left(h_{k}(i)\right)\right)\right| \\
& +\frac{1}{n b(n+\tau)} \\
& +\frac{1}{n b(n+\tau)} \\
& \cdot \sum_{i=n+\tau}^{\infty} \sum_{s=i}^{\infty} \sum_{t=s}^{\infty}\left[\left|f\left(t, y\left(f_{1}(t)\right), \ldots, y\left(f_{k}(t)\right)\right)\right|\right. \\
& +|d(t)|] \\
& \geq \frac{L}{b_{*}}-\frac{A_{*}}{b_{*}}+\frac{c^{*}}{b^{*}}\left(1+\frac{\tau}{T}\right) \\
& +\frac{1}{T b^{*}} \sum_{i=T+\tau}^{\infty} H_{i}+\frac{1}{T b^{*}} \sum_{i=T+\tau}^{\infty} \sum_{s=i}^{\infty} G_{s} \\
& +\frac{1}{T b^{*}} \sum_{i=T+\tau}^{\infty} \sum_{s=i}^{\infty} \sum_{t=s}^{\infty}\left[F_{t}+|d(t)|\right] \\
& \geq \frac{L}{b_{*}}-\frac{A_{*}}{b_{*}}+\frac{c^{*}}{b^{*}}\left(1+\frac{\tau}{T}\right) \\
& +\frac{1}{b^{*}} \min \left\{L-b^{*} B-\left(1+\frac{\tau}{T}\right)\left(B^{*}+c^{*}\right),\right. \\
& \left.b^{*} A+\frac{b^{*} A_{*}}{b_{*}}-c^{*}\left(1+\frac{\tau}{T}\right)-\frac{b^{*}}{b_{*}} L\right\} \\
& \geq A \geq A(n), \quad n \geq T, \\
& \frac{\left(S_{L} x\right)(n)+\left(G_{L} y\right)(n)}{n}=\frac{n}{T} \cdot \frac{\left(S_{L} x\right)(T)+\left(G_{L} y\right)(T)}{n} \\
& \geq A(T), \quad \beta \leq n<T, \\
& \left|\frac{\left(S_{L} x\right)(n)-\left(S_{L} y\right)(n)}{n}\right|=\frac{n+\tau}{n|b(n+\tau)|}\left|\frac{x(n+\tau)-y(n+\tau)}{n+\tau}\right| \\
& \leq \theta\|x-y\|, \quad n \geq T, \\
& \left|\frac{\left(S_{L} x\right)(n)-\left(S_{L} y\right)(n)}{n}\right|=\frac{n}{T}\left|\frac{\left(S_{L} x\right)(T)-\left(S_{L} y\right)(T)}{n}\right| \\
& \leq \theta\|x-y\|, \quad \beta \leq n<T, \\
& \left|\frac{\left(G_{L} y\right)(n)}{n}\right| \\
& =\mid \frac{1}{n b(n+\tau)} \sum_{i=n+\tau}^{\infty} h\left(i, y\left(h_{1}(i)\right), \ldots, y\left(h_{k}(i)\right)\right) \\
& -\frac{1}{n b(n+\tau)} \sum_{i=n+\tau}^{\infty} \sum_{s=i}^{\infty} g\left(s, y\left(g_{1}(s)\right), \ldots, y\left(g_{k}(s)\right)\right)
\end{aligned}
$$




$$
\begin{aligned}
& +\frac{1}{n b(n+\tau)} \\
& \cdot \sum_{i=n+\tau}^{\infty} \sum_{s=i}^{\infty} \sum_{t=s}^{\infty}\left[f\left(t, y\left(f_{1}(t)\right), \ldots, y\left(f_{k}(t)\right)\right)-d(t)\right] \\
& \leq \frac{1}{n|b(n+\tau)|} \sum_{i=n+\tau}^{\infty}\left|h\left(i, y\left(h_{1}(i)\right), \ldots, y\left(h_{k}(i)\right)\right)\right| \\
& +\frac{1}{n|b(n+\tau)|} \sum_{i=n+\tau}^{\infty} \sum_{s=i}^{\infty}\left|g\left(s, y\left(g_{1}(s)\right), \ldots, y\left(g_{k}(s)\right)\right)\right| \\
& +\frac{1}{n|b(n+\tau)|} \\
& \cdot \sum_{i=n+\tau}^{\infty} \sum_{s=i}^{\infty} \sum_{t=s}^{\infty}\left[\left|f\left(t, y\left(f_{1}(t)\right), \ldots, y\left(f_{k}(t)\right)\right)\right|+|d(t)|\right] \\
& \leq \frac{1}{T\left|b^{*}\right|} \sum_{i=T+\tau}^{\infty} H_{i}+\frac{1}{T\left|b^{*}\right|} \sum_{i=T+\tau}^{\infty} \sum_{s=i}^{\infty} G_{s}+\frac{1}{T\left|b^{*}\right|} \\
& \sum_{i=T+\tau}^{\infty} \sum_{s=i}^{\infty} \sum_{t=s}^{\infty}\left[F_{t}+|d(t)|\right] \\
& \leq-\frac{1}{b^{*}} \min \left\{L-b^{*} B-\left(1+\frac{\tau}{T}\right)\left(B^{*}+c^{*}\right),\right. \\
& \left.b^{*} A+\frac{b^{*} A_{*}}{b_{*}}-c^{*}\left(1+\frac{\tau}{T}\right)-\frac{b^{*}}{b_{*}} L\right\} \\
& \leq B, \quad n \geq T, \\
& \left|\frac{\left(G_{L} y\right)(n)}{n}\right|=\frac{n}{T}\left|\frac{\left(G_{L} y\right)(T)}{n}\right| \leq B, \quad \beta \leq n<T,
\end{aligned}
$$

which yield (23), (25), and (48).

Next we show that $G_{L}$ is completely continuous. Let $y^{w}=$ $\left\{y^{w}(n)\right\}_{n \in \mathbb{N}_{\beta}}$ and $y=\{y(n)\}_{n \in \mathbb{N}_{\beta}} \in \Omega\left(A_{*}, B^{*}, T\right)$ with (27). Using (16), (17), (27), and the continuity of $f, g$, and $h$, we know that for given $\varepsilon>0$, there exist $T_{1}, T_{2}, T_{3}$, and $T_{4} \in \mathbb{N}$ with $T_{4}>T_{3}>T_{2}>T_{1}>T+\tau$ satisfying

$$
\begin{aligned}
\frac{1}{T\left|b^{*}\right|} \max \{ & \sum_{i=T_{1}+1}^{\infty} H_{i}+\sum_{i=T_{1}+1}^{\infty} \sum_{s=i}^{\infty} G_{s} \\
& +\sum_{i=T_{1}+1}^{\infty} \sum_{s=i}^{\infty} \sum_{t=s}^{\infty}\left[F_{t}+|d(t)|\right] \\
& \sum_{i=T+\tau}^{T_{1}} \sum_{s=T_{2}+1}^{\infty} G_{s}+\sum_{i=T+\tau} \sum_{s=T_{2}+1}^{\infty} \sum_{t=s}^{\infty} F_{t} \\
& \left.+\sum_{i=T+\tau}^{T_{1}} \sum_{s=i}^{T_{2}} \sum_{s=T_{3}+1}^{\infty} F_{t}\right\}<\frac{\varepsilon}{16}
\end{aligned}
$$

$$
\begin{aligned}
& \frac{1}{T\left|b^{*}\right|} \max \left\{\sum_{i=T+\tau}^{T_{1}} \mid h\left(i, y^{w}\left(h_{1}(i)\right), \ldots, y^{w}\left(h_{k}(i)\right)\right)\right. \\
& -h\left(i, y\left(h_{1}(i)\right), \ldots, y\left(h_{k}(i)\right)\right) \mid \\
& \sum_{i=T+\tau}^{T_{1}} \sum_{s=i}^{T_{2}} \mid g\left(s, y^{w}\left(g_{1}(s)\right), \ldots, y^{w}\left(g_{k}(s)\right)\right) \\
& -g\left(s, y\left(g_{1}(s)\right), \ldots, y\left(g_{k}(s)\right)\right) \mid \\
& \sum_{i=T+\tau}^{T_{1}} \sum_{s=i}^{T_{2}} \sum_{t=s}^{T_{3}} \mid f\left(t, y^{w}\left(f_{1}(t)\right), \ldots, y^{w}\left(f_{k}(t)\right)\right) \\
& \left.-f\left(t, y\left(f_{1}(t)\right), \ldots, y\left(f_{k}(t)\right)\right) \mid\right\} \\
& <\frac{\varepsilon}{16}, \quad w \geq T_{4}
\end{aligned}
$$

Combining (15), (47), and (74), we infer that

$$
\begin{aligned}
& \left\|G_{L} y^{w}-G_{L} y\right\| \\
& =\sup \left\{\frac{\left|\left(G_{L} y^{w}\right)(n)-\left(G_{L} y\right)(n)\right|}{n}: n \in \mathbb{N}_{\beta}\right\} \\
& =\max \left\{\sup \left\{\frac{n}{T} \cdot \frac{\left|\left(G_{L} y^{w}\right)(T)-\left(G_{L} y\right)(T)\right|}{n}: \beta \leq n<T\right\},\right. \\
& \left.\quad \sup \left\{\frac{\left|\left(G_{L} y^{w}\right)(n)-\left(G_{L} y\right)(n)\right|}{n}: n \in \mathbb{N}_{T}\right\}\right\}
\end{aligned}
$$$$
\leq \sup \left\{\frac{1}{n|b(n+\tau)|}\right.
$$$$
\cdot \sum_{i=n+\tau}^{\infty} \mid h\left(i, y^{w}\left(h_{1}(i)\right), \ldots, y^{w}\left(h_{k}(i)\right)\right)
$$$$
-h\left(i, y\left(h_{1}(i)\right), \ldots, y\left(h_{k}(i)\right)\right)
$$$$
+\frac{1}{n|b(n+\tau)|}
$$$$
\cdot \sum_{i=n+\tau}^{\infty} \sum_{s=i}^{\infty} \mid g\left(s, y^{w}\left(g_{1}(s)\right), \ldots, y^{w}\left(g_{k}(s)\right)\right)
$$$$
-g\left(s, y\left(g_{1}(s)\right), \ldots, y\left(g_{k}(s)\right)\right) \mid
$$$$
+\frac{1}{n|b(n+\tau)|}
$$ 


$$
\begin{aligned}
& \cdot \sum_{i=n+\tau}^{\infty} \sum_{s=i}^{\infty} \sum_{t=s}^{\infty} \mid f\left(t, y^{w}\left(f_{1}(t)\right), \ldots, y^{w}\left(f_{k}(t)\right)\right) \\
& \cdot \sum_{i=T+\tau}^{T_{1}} \sum_{s=i}^{T_{2}} \sum_{t=T_{3}+1}^{\infty} \mid f\left(t, y^{w}\left(f_{1}(t)\right), \ldots, y^{w}\left(f_{k}(t)\right)\right) \\
& -f\left(t, y\left(f_{1}(t)\right), \ldots, y\left(f_{k}(t)\right)\right) \mid: \\
& \left.n \in \mathbb{N}_{T}\right\} \\
& \leq \frac{1}{T\left|b^{*}\right|} \sum_{i=T+\tau}^{T_{1}} \mid h\left(i, y^{w}\left(h_{1}(i)\right), \ldots, y^{w}\left(h_{k}(i)\right)\right) \\
& -h\left(i, y\left(h_{1}(i)\right), \ldots, y\left(h_{k}(i)\right)\right) \\
& +\frac{1}{T\left|b^{*}\right|} \sum_{i=T_{1}+1}^{\infty} \mid h\left(i, y^{w}\left(h_{1}(i)\right), \ldots, y^{w}\left(h_{k}(i)\right)\right) \\
& -h\left(i, y\left(h_{1}(i)\right), \ldots, y\left(h_{k}(i)\right)\right) \mid \\
& +\frac{1}{T\left|b^{*}\right|} \\
& \cdot \sum_{i=T+\tau}^{T_{1}} \sum_{s=i}^{T_{2}} \mid g\left(s, y^{w}\left(g_{1}(s)\right), \ldots, y^{w}\left(g_{k}(s)\right)\right) \\
& -g\left(s, y\left(g_{1}(s)\right), \ldots, y\left(g_{k}(s)\right)\right) \\
& +\frac{1}{T\left|b^{*}\right|} \\
& \cdot \sum_{i=T+\tau}^{T_{1}} \sum_{s=T_{2}+1}^{\infty} \mid g\left(s, y^{w}\left(g_{1}(s)\right), \ldots, y^{w}\left(g_{k}(s)\right)\right) \\
& -g\left(s, y\left(g_{1}(s)\right), \ldots, y\left(g_{k}(s)\right)\right) \\
& +\frac{1}{T\left|b^{*}\right|} \\
& \cdot \sum_{i=T_{1}+1}^{\infty} \sum_{s=i}^{\infty} \lg \left(s, y^{w}\left(g_{1}(s)\right), \ldots, y^{w}\left(g_{k}(s)\right)\right) \\
& -f\left(t, y\left(f_{1}(t)\right), \ldots, y\left(f_{k}(t)\right)\right) \mid \\
& +\frac{1}{T\left|b^{*}\right|} \\
& \cdot \sum_{i=T+\tau}^{T_{1}} \sum_{s=T_{2}+1}^{\infty} \sum_{t=s}^{\infty} \mid f\left(t, y^{w}\left(f_{1}(t)\right), \ldots, y^{w}\left(f_{k}(t)\right)\right) \\
& -f\left(t, y\left(f_{1}(t)\right), \ldots, y\left(f_{k}(t)\right)\right) \mid \\
& +\frac{1}{T\left|b^{*}\right|} \\
& \cdot \sum_{i=T_{1}+1}^{\infty} \sum_{s=i}^{\infty} \sum_{t=s}^{\infty} \mid f\left(t, y^{w}\left(f_{1}(t)\right), \ldots, y^{w}\left(f_{k}(t)\right)\right) \\
& -f\left(t, y\left(f_{1}(t)\right), \ldots, y\left(f_{k}(t)\right)\right) \\
& <\frac{\varepsilon}{16}+\frac{2}{T\left|b^{*}\right|} \sum_{i=T_{1}+1}^{\infty} H_{i}+\frac{\varepsilon}{16} \\
& +\frac{2}{T\left|b^{*}\right|} \sum_{i=T+\tau}^{T_{1}} \sum_{s=T_{2}+1}^{\infty} G_{s}+\frac{2}{T\left|b^{*}\right|} \sum_{i=T_{1}+1}^{\infty} \sum_{s=i}^{\infty} G_{s} \\
& +\frac{\varepsilon}{16}+\frac{2}{T\left|b^{*}\right|} \sum_{i=T+\tau}^{T_{1}} \sum_{s=i}^{T_{2}} \sum_{t=T_{3}+1}^{\infty} F_{t} \\
& +\frac{2}{T\left|b^{*}\right|} \sum_{i=T+\tau}^{T_{1}} \sum_{s=T_{2}+1}^{\infty} \sum_{t=s}^{\infty} F_{t} \\
& +\frac{2}{T\left|b^{*}\right|} \sum_{i=T_{1}+1}^{\infty} \sum_{s=i}^{\infty} \sum_{t=s}^{\infty} F_{t}<\varepsilon, \quad w \geq T_{4},
\end{aligned}
$$$$
-g\left(s, y\left(g_{1}(s)\right), \ldots, y\left(g_{k}(s)\right)\right) \mid
$$$$
+\frac{1}{T\left|b^{*}\right|}
$$$$
\cdot \sum_{i=T+\tau}^{T_{1}} \sum_{s=i}^{T_{2}} \sum_{t=s}^{T_{3}} \mid f\left(t, y^{w}\left(f_{1}(t)\right), \ldots, y^{w}\left(f_{k}(t)\right)\right)
$$$$
-f\left(t, y\left(f_{1}(t)\right), \ldots, y\left(f_{k}(t)\right)\right)
$$$$
+\frac{1}{T\left|b^{*}\right|}
$$

which implies that $G_{L}$ is continuous in $\Omega\left(A_{*}, B^{*}, T\right)$.

It follows from (47) and (68) that for any $x=\{x(n)\}_{n \in \mathbb{N}_{\beta}} \in$ $\Omega\left(A_{*}, B^{*}, T\right)$ and $t_{1}, t_{2} \geq T_{4}$

$$
\begin{aligned}
& \left|\frac{\left(G_{L} y\right)\left(t_{2}\right)}{t_{2}}-\frac{\left(G_{L} y\right)\left(t_{1}\right)}{t_{1}}\right| \\
& =\mid \frac{1}{t_{2} b(n+\tau)} \sum_{i=t_{2}+\tau}^{\infty} h\left(i, y\left(h_{1}(i)\right), \ldots, y\left(h_{k}(i)\right)\right) \\
& \quad-\frac{1}{t_{1} b(n+\tau)} \sum_{i=t_{1}+\tau}^{\infty} h\left(i, y\left(h_{1}(i)\right), \ldots, y\left(h_{k}(i)\right)\right)
\end{aligned}
$$




$$
\begin{array}{r}
-\frac{1}{t_{2} b(n+\tau)} \sum_{i=t_{2}+\tau}^{\infty} \sum_{s=i}^{\infty} g\left(s, y\left(g_{1}(s)\right), \ldots, y\left(g_{k}(s)\right)\right) \\
+\frac{1}{t_{1} b(n+\tau)} \sum_{i=t_{1}+\tau}^{\infty} \sum_{s=i}^{\infty} g\left(s, y\left(g_{1}(s)\right), \ldots, y\left(g_{k}(s)\right)\right) \\
+\frac{1}{t_{2} b(n+\tau)} \\
\cdot \sum_{i=t_{2}+\tau}^{\infty} \sum_{s=i}^{\infty} \sum_{i=s}^{\infty}\left[f\left(t, y\left(f_{1}(t)\right), \ldots, y\left(f_{k}(t)\right)\right)-d(t)\right] \\
\quad-\frac{1}{t_{1} b(n+\tau)} \\
\quad \cdot \sum_{i=t_{1}+\tau}^{\infty} \sum_{s=i}^{\infty} \sum_{t=s}^{\infty}\left[f\left(t, y\left(f_{1}(t)\right), \ldots, y\left(f_{k}(t)\right)\right)-d(t)\right] \\
\frac{2}{T_{4}\left|b^{*}\right|} \\
\cdot\left(\sum_{i=T_{4}+\tau}^{\infty} H_{i}+\sum_{i=T_{4}+\tau}^{\infty} \sum_{s=i}^{\infty} G_{s}+\sum_{i=T_{4}+\tau}^{\infty} \sum_{s=i}^{\infty} \sum_{t=s}^{\infty}\left[F_{t}+|d(t)|\right]\right)
\end{array}
$$$$
<\varepsilon
$$

which means that $G_{L}\left(\Omega\left(A_{*}, B^{*}, T\right)\right)$ is uniformly Cauchy, which together with (25) and Lemma 1 yields that $G_{L}\left(\Omega\left(A_{*}\right.\right.$, $\left.B^{*}, T\right)$ ) is relatively compact. That is, $G_{L}$ is completely continuous in $\Omega\left(A_{*}, B^{*}, T\right)$. Thus (25), (48), and Lemma 2 ensure that the mapping $S_{L}+G_{L}$ has a fixed point $x=\{x(n)\}_{n \in \mathbb{N}_{\beta}} \in$ $\Omega\left(A_{*}, B^{*}, T\right)$, which together with (46) and (47) implies that

$$
\begin{aligned}
x(n)= & \frac{n L}{b(n+\tau)}-\frac{x(n+\tau)}{b(n+\tau)}-\frac{c(n+\tau)}{b(n+\tau)}+\frac{1}{b(n+\tau)} \\
& \cdot \sum_{i=n+\tau}^{\infty} h\left(i, x\left(h_{1}(i)\right), \ldots, x\left(h_{k}(i)\right)\right)-\frac{1}{b(n+\tau)} \\
& \cdot \sum_{i=n+\tau}^{\infty} \sum_{s=i}^{\infty} g\left(s, x\left(g_{1}(s)\right), \ldots, x\left(g_{k}(s)\right)\right)+\frac{1}{b(n+\tau)} \\
& \cdot \sum_{i=n+\tau}^{\infty} \sum_{s=i}^{\infty} \sum_{t=s}^{\infty}\left[f\left(t, x\left(f_{1}(t)\right), \ldots, x\left(f_{k}(t)\right)\right)-d(t)\right],
\end{aligned}
$$$$
n \geq T \text {, }
$$

That is, $x=\{x(n)\}_{n \in \mathbb{N}_{\beta}}$ is a positive solution of (4). The rest of the proof is similar to that of Theorem 6 and is omitted. This completes the proof.
Theorem 9. Assume that there exist three nonnegative sequences $\left\{F_{n}\right\}_{n \in \mathbb{N}_{n_{0}}},\left\{G_{n}\right\}_{n \in \mathbb{N}_{n_{0}}}$, and $\left\{H_{n}\right\}_{n \in \mathbb{N}_{n_{0}}}$ satisfying (15),

$$
\begin{gathered}
\lim _{n \rightarrow \infty} \frac{1}{n} \max \left\{\sum_{t=n}^{\infty} t H_{t}, \sum_{t=n}^{\infty} t^{2} G_{t}\right\}=0, \\
\lim _{n \rightarrow \infty} \frac{1}{n} \sum_{t=n}^{\infty} t^{3} \max \left\{F_{t},|d(t)|\right\}=0, \\
\lim _{n \rightarrow \infty} \frac{1}{n} \sum_{i=1}^{\infty}|c(n+i \tau)|=0, \\
b(n)=-1, \quad n \in \mathbb{N}_{n_{0}} .
\end{gathered}
$$

Then

(i) equation (4) possesses a positive solution $x=$ $\{x(n)\}_{n \in \mathbb{N}_{\beta}} \in l_{\beta}^{\infty}$ with (19) and

$$
\lim _{n \rightarrow \infty} \frac{x(n)-x(n-\tau)+c(n)}{n}=0 ;
$$

(ii) equation (4) possesses uncountably many positive solutions in $l_{\beta}^{\infty}$.

Proof. (i) Let $L \in(A, B)$. It follows from (78)-(80) that there exists $T \geq n_{0}+\tau+\alpha$ satisfying

$$
\begin{gathered}
\frac{1}{n} \sum_{i=1}^{\infty}|c(n+i \tau)|<\frac{1}{2} \min \{B-L, L-A\}, \quad n \in \mathbb{N}_{T}, \\
\frac{1}{T} \sum_{t=T}^{\infty} t H_{t}+\frac{1}{T} \sum_{t=T}^{\infty} t^{2} G_{t}+\frac{1}{T} \sum_{t=T}^{\infty} t^{3}\left[F_{t}+|d(t)|\right] \\
<\frac{1}{2} \min \{B-L, L-A\} .
\end{gathered}
$$

Define a mapping $S_{L}: \Omega\left(A_{*}, B^{*}, T\right) \rightarrow l_{\beta}^{\infty}$ by

$$
\begin{aligned}
& \left(S_{L} x\right)(n) \\
& \qquad \begin{array}{c}
n L+\sum_{i=1}^{\infty} c(n+i \tau) \\
-\sum_{i=1}^{\infty} \sum_{t=n+i \tau}^{\infty} h\left(t, x\left(h_{1}(t)\right), \ldots, x\left(h_{k}(t)\right)\right) \\
+\sum_{i=1}^{\infty} \sum_{s=n+i \tau}^{\infty} \sum_{t=s}^{\infty} g\left(t, x\left(g_{1}(t)\right), \ldots, x\left(g_{k}(t)\right)\right) \\
-\sum_{p=1}^{\infty} \sum_{i=n+p \tau}^{\infty} \sum_{s=i}^{\infty} \sum_{t=s}^{\infty}\left[f\left(t, x\left(f_{1}(t)\right), \ldots, x\left(f_{k}(t)\right)\right)\right. \\
\quad-d(t)], \\
n \\
\frac{n}{T}\left(S_{L} x\right)(T), \quad \beta \leq n<T,
\end{array}
\end{aligned}
$$

for any $x=\{x(n)\}_{n \in \mathbb{N}_{\beta}} \in \Omega\left(A_{*}, B^{*}, T\right)$. 
Now we show that

$$
\begin{gathered}
S_{L} x \in \Omega\left(A_{*}, B^{*}, T\right), \quad x \in \Omega\left(A_{*}, B^{*}, T\right) ; \\
\left\|S_{L} x\right\| \leq B, \quad x \in \Omega\left(A_{*}, B^{*}, T\right) .
\end{gathered}
$$

It follows from (15), (83)-(85), and Lemma 4 that for any $x=$ $\{x(n)\}_{n \in \mathbb{N}_{\beta}} \in \Omega\left(A_{*}, B^{*}, T\right)$

$$
\begin{aligned}
& \left|\frac{\left(S_{L} x\right)(n)}{n}-L\right| \\
& =\mid \frac{1}{n} \sum_{i=1}^{\infty} c(n+i \tau) \\
& -\frac{1}{n} \sum_{i=1}^{\infty} \sum_{t=n+i \tau}^{\infty} h\left(t, x\left(h_{1}(t)\right), \ldots, x\left(h_{k}(t)\right)\right) \\
& +\frac{1}{n} \sum_{i=1}^{\infty} \sum_{s=n+i \tau}^{\infty} \sum_{t=s}^{\infty} g\left(t, x\left(g_{1}(t)\right), \ldots, x\left(g_{k}(t)\right)\right) \\
& -\frac{1}{n} \sum_{p=1}^{\infty} \sum_{i=n+p \tau}^{\infty} \sum_{s=i}^{\infty} \sum_{t=s}^{\infty}\left[f\left(t, x\left(f_{1}(t)\right), \ldots, x\left(f_{k}(t)\right)\right)\right. \\
& -d(t)] \\
& \leq \frac{1}{n} \sum_{i=1}^{\infty}|c(n+i \tau)| \\
& +\frac{1}{n} \sum_{i=1}^{\infty} \sum_{t=n+i \tau}^{\infty}\left|h\left(t, x\left(h_{1}(t)\right), \ldots, x\left(h_{k}(t)\right)\right)\right| \\
& +\frac{1}{n} \sum_{i=1}^{\infty} \sum_{s=n+i \tau}^{\infty} \sum_{t=s}^{\infty}\left|g\left(t, x\left(g_{1}(t)\right), \ldots, x\left(g_{k}(t)\right)\right)\right| \\
& +\frac{1}{n} \sum_{p=1}^{\infty} \sum_{i=n+p \tau}^{\infty} \sum_{s=i}^{\infty} \sum_{t=s}^{\infty}\left[\left|f\left(t, x\left(f_{1}(t)\right), \ldots, x\left(f_{k}(t)\right)\right)\right|\right. \\
& +|d(t)|] \\
& <\frac{1}{2} \min \{B-L, L-A\} \\
& +\frac{1}{T} \sum_{t=T}^{\infty} t H_{t}+\frac{1}{T} \sum_{t=T}^{\infty} t^{2} G_{t}+\frac{1}{T} \sum_{t=T}^{\infty} t^{3}\left[F_{t}+|d(t)|\right] \\
& <\min \{B-L, L-A\}, \quad n \geq T, \\
& \left|\frac{\left(S_{L} x\right)(n)}{n}-L\right|=\left|\frac{n}{T} \cdot \frac{\left(S_{L} x\right)(T)}{n}-L\right| \\
& <\min \{B-L, L-A\}, \quad \beta \leq n<T,
\end{aligned}
$$

which yields that

$$
\begin{aligned}
A(n) & \leq A \leq L-\min \{B-L, L-A\}<\frac{\left(S_{L} x\right)(n)}{n} \\
& <L+\min \{B-L, L-A\} \leq B \leq B(n), \quad n \in \mathbb{N}_{\beta} ;
\end{aligned}
$$

that is, (86) and (87) hold.

Next we show that $S_{L}$ is continuous and $S_{L}\left(\Omega\left(A_{*}, B^{*}, T\right)\right)$ is uniformly Cauchy. Let $x^{w}=\left\{x^{w}(n)\right\}_{n \in \mathbb{N}_{\beta}}$ and $x=$ $\{x(n)\}_{n \in \mathbb{N}_{\beta}} \in \Omega\left(A_{*}, B^{*}, T\right)$ with

$$
\lim _{w \rightarrow \infty} x^{w}=x
$$

Using (15), (78), and (80) the continuity of $f, g$, and $h$, we know that for given $\varepsilon>0$, there exist $T_{2}>T_{1}>T$ satisfying

$$
\begin{aligned}
& \frac{1}{n} \sum_{i=1}^{\infty}|c(n+i \tau)|<\frac{\varepsilon}{16}, \quad \forall n \in \mathbb{N}_{T_{1}}, \\
& \frac{1}{T} \max \left\{\sum_{t=T}^{T_{1}} t \mid h\left(t, x^{w}\left(h_{1}(t)\right), \ldots, x^{w}\left(h_{k}(t)\right)\right)\right. \\
& -h\left(t, x\left(h_{1}(t)\right), \ldots, x\left(h_{k}(t)\right)\right) \mid \text {, } \\
& \sum_{t=T}^{T_{1}} t^{2} \lg \left(t, x^{w}\left(g_{1}(t)\right), \ldots, x^{w}\left(g_{k}(t)\right)\right) \\
& -g\left(t, x\left(g_{1}(t)\right), \ldots, x\left(g_{k}(t)\right)\right) \mid, \\
& \sum_{t=T}^{T_{1}} t^{3} \mid f\left(t, x^{w}\left(f_{1}(t)\right), \ldots, x^{w}\left(f_{k}(t)\right)\right) \\
& \left.-f\left(t, x\left(f_{1}(t)\right), \ldots, x\left(f_{k}(t)\right)\right) \mid\right\} \\
& <\frac{\varepsilon}{16}, \quad w \geq T_{2} \text {, } \\
& \frac{1}{T}\left(\sum_{t=T_{1}+1}^{\infty} t H_{t}+\sum_{t=T_{1}+1}^{\infty} t^{2} G_{t}+\sum_{t=T_{1}+1}^{\infty} t^{3}\left[F_{t}+|d(t)|\right]\right) \\
& <\frac{\varepsilon}{16} \text {. }
\end{aligned}
$$

Combining (15), (91), and Lemma 4, we infer that

$$
\begin{aligned}
& \left\|S_{L} x^{w}-S_{L} x\right\| \\
& =\sup \left\{\left|\frac{\left(S_{L} x^{w}\right)(n)-\left(S_{L} x\right)(n)}{n}\right|: n \in \mathbb{N}_{\beta}\right\} \\
& =\max \left\{\sup \left\{\left|\frac{n}{T} \frac{\left(S_{L} x^{w}\right)(T)-\left(S_{L} x\right)(T)}{n}\right|: \beta \leq n<T\right\},\right.
\end{aligned}
$$




$$
\begin{aligned}
& \left.\sup \left\{\left|\frac{\left(S_{L} x^{w}\right)(n)-\left(S_{L} x\right)(n)}{n}\right|: n \in \mathbb{N}_{T}\right\}\right\} \\
& \leq \sup \left\{\frac{1}{n} \sum_{i=1}^{\infty} \sum_{t=n+i \tau}^{\infty} \mid h\left(t, x^{w}\left(h_{1}(t)\right), \ldots, x^{w}\left(h_{k}(t)\right)\right)\right. \\
& -h\left(t, x\left(h_{1}(t)\right), \ldots, x\left(h_{k}(t)\right)\right) \mid \\
& +\frac{1}{n} \sum_{i=1}^{\infty} \sum_{s=n+i \tau}^{\infty} \sum_{t=s}^{\infty} \mid g\left(t, x^{w}\left(g_{1}(t)\right), \ldots, x^{w}\left(g_{k}(t)\right)\right) \\
& -g\left(t, x\left(g_{1}(t)\right), \ldots, x\left(g_{k}(t)\right)\right) \\
& +\frac{1}{n} \sum_{p=1}^{\infty} \sum_{i=n+p \tau}^{\infty} \sum_{s=i}^{\infty} \sum_{t=s}^{\infty} \mid f\left(t, x^{w}\left(f_{1}(t)\right), \ldots\right. \\
& \left.x^{w}\left(f_{k}(t)\right)\right) \\
& -f\left(t, x\left(f_{1}(t)\right), \ldots,\right. \\
& \left.x\left(f_{k}(t)\right)\right) \mid: \\
& \left.n \in \mathbb{N}_{T}\right\} \\
& \leq \frac{1}{T} \sum_{t=T+\tau}^{\infty} t \mid h\left(t, x^{w}\left(h_{1}(t)\right), \ldots, x^{w}\left(h_{k}(t)\right)\right) \\
& +\frac{1}{T} \sum_{t=T}^{T_{1}} t^{2} \mid g\left(t, x^{w}\left(g_{1}(t)\right), \ldots, x^{w}\left(g_{k}(t)\right)\right) \\
& -g\left(t, x\left(g_{1}(t)\right), \ldots, x\left(g_{k}(t)\right)\right) \\
& +\frac{1}{T} \sum_{t=T_{1}+1}^{\infty} t^{2} \mid g\left(t, x^{w}\left(g_{1}(t)\right), \ldots, x^{w}\left(g_{k}(t)\right)\right) \\
& -g\left(t, x\left(g_{1}(t)\right), \ldots, x\left(g_{k}(t)\right)\right) \mid \\
& +\frac{1}{T} \sum_{t=T}^{T_{1}} t^{3} \mid f\left(t, x^{w}\left(f_{1}(t)\right), \ldots, x^{w}\left(f_{k}(t)\right)\right) \\
& -f\left(t, x\left(f_{1}(t)\right), \ldots, x\left(f_{k}(t)\right)\right) \mid \\
& +\frac{1}{T} \sum_{t=T_{1}+1}^{\infty} t^{3} \mid f\left(t, x^{w}\left(f_{1}(t)\right), \ldots, x^{w}\left(f_{k}(t)\right)\right) \\
& -f\left(t, x\left(f_{1}(t)\right), \ldots, x\left(f_{k}(t)\right)\right) \mid \\
& <\frac{\varepsilon}{16}+\frac{2}{T} \sum_{t=T_{1}+1}^{\infty} t H_{t}+\frac{\varepsilon}{16}+\frac{2}{T} \sum_{t=T_{1}+1}^{\infty} t^{2} G_{t} \\
& +\frac{\varepsilon}{16}+\frac{2}{T} \sum_{i=T_{1}+1}^{\infty} t^{3} F_{t}<\varepsilon, \quad w \geq T_{2}
\end{aligned}
$$$$
-h\left(t, x\left(h_{1}(t)\right), \ldots, x\left(h_{k}(t)\right)\right)
$$$$
+\frac{1}{T} \sum_{t=T+\tau}^{\infty} t^{2} \mid g\left(t, x^{w}\left(g_{1}(t)\right), \ldots, x^{w}\left(g_{k}(t)\right)\right)
$$$$
-g\left(t, x\left(g_{1}(t)\right), \ldots, x\left(g_{k}(t)\right)\right)
$$$$
+\frac{1}{T} \sum_{t=T+\tau}^{\infty} t^{3} \mid f\left(t, x^{w}\left(f_{1}(t)\right), \ldots, x^{w}\left(f_{k}(t)\right)\right)
$$$$
-f\left(t, x\left(f_{1}(t)\right), \ldots, x\left(f_{k}(t)\right)\right)
$$$$
\leq \frac{1}{T} \sum_{t=T}^{T_{1}} t \mid h\left(t, x^{w}\left(h_{1}(t)\right), \ldots, x^{w}\left(h_{k}(t)\right)\right)
$$$$
-h\left(t, x\left(h_{1}(t)\right), \ldots, x\left(h_{k}(t)\right)\right)
$$$$
+\frac{1}{T} \sum_{t=T_{1}+1}^{\infty} t \mid h\left(t, x^{w}\left(h_{1}(t)\right), \ldots, x^{w}\left(h_{k}(t)\right)\right)
$$$$
-h\left(t, x\left(h_{1}(t)\right), \ldots, x\left(h_{k}(t)\right)\right) \mid
$$

which implies that $S_{L}$ is continuous in $\Omega\left(A_{*}, B^{*}, T\right)$. It follows from (80), (85), and Lemma 4 that for any $x=\{x(n)\}_{n \in \mathbb{N}_{\beta}} \in$ $\Omega\left(A_{*}, B^{*}, T\right)$ and $t_{1}>t_{2} \geq T_{2}$

$$
\begin{aligned}
& \left|\frac{\left(S_{L} x\right)\left(t_{1}\right)}{t_{1}}-\frac{\left(S_{L} x\right)\left(t_{2}\right)}{t_{2}}\right| \\
& =\mid \frac{1}{t_{1}} \sum_{i=1}^{\infty} c\left(t_{1}+i \tau\right)-\frac{1}{t_{2}} \sum_{i=1}^{\infty} c\left(t_{2}+i \tau\right) \\
& \quad-\frac{1}{t_{1}} \sum_{i=1}^{\infty} \sum_{t=t_{1}+i \tau}^{\infty} h\left(t, x\left(h_{1}(t)\right), \ldots, x\left(h_{k}(t)\right)\right) \\
& \quad+\frac{1}{t_{2}} \sum_{i=1}^{\infty} \sum_{t=t_{2}+i \tau}^{\infty} h\left(t, x\left(h_{1}(t)\right), \ldots, x\left(h_{k}(t)\right)\right) \\
& \quad+\frac{1}{t_{1}} \sum_{i=1}^{\infty} \sum_{s=t_{1}+i \tau}^{\infty} \sum_{t=s}^{\infty} g\left(t, x\left(g_{1}(t)\right), \ldots, x\left(g_{k}(t)\right)\right) \\
& \quad-\frac{1}{t_{2}} \sum_{i=1}^{\infty} \sum_{s=t_{2}+i \tau}^{\infty} \sum_{t=s}^{\infty} g\left(t, x\left(g_{1}(t)\right), \ldots, x\left(g_{k}(t)\right)\right)
\end{aligned}
$$




$$
\begin{aligned}
& -\frac{1}{t_{1}} \sum_{p=1}^{\infty} \sum_{i=t_{1}+p \tau}^{\infty} \sum_{s=i}^{\infty} \sum_{t=s}^{\infty}\left[f\left(t, x\left(f_{1}(t)\right), \ldots, x\left(f_{k}(t)\right)\right)\right. \\
& -d(t)] \\
& +\frac{1}{t_{2}} \sum_{p=1}^{\infty} \sum_{i=t_{2}+p \tau}^{\infty} \sum_{s=i}^{\infty} \sum_{t=s}^{\infty}\left[f\left(t, x\left(f_{1}(t)\right), \ldots, x\left(f_{k}(t)\right)\right)\right. \\
& -d(t)] \\
& \leq \frac{1}{t_{1}} \sum_{i=1}^{\infty}\left|c\left(t_{1}+i \tau\right)\right|+\frac{1}{t_{2}} \sum_{i=1}^{\infty}\left|c\left(t_{2}+i \tau\right)\right| \\
& +\frac{1}{t_{1}} \sum_{t=t_{1}}^{\infty} t\left|h\left(t, x\left(h_{1}(t)\right), \ldots, x\left(h_{k}(t)\right)\right)\right| \\
& +\frac{1}{t_{2}} \sum_{t=t_{2}}^{\infty} t\left|h\left(t, x\left(h_{1}(t)\right), \ldots, x\left(h_{k}(t)\right)\right)\right| \\
& +\frac{1}{t_{1}} \sum_{t=t_{1}}^{\infty} t^{2}\left|g\left(t, x\left(g_{1}(t)\right), \ldots, x\left(g_{k}(t)\right)\right)\right| \\
& +\frac{1}{t_{2}} \sum_{t=t_{2}}^{\infty} t^{2}\left|g\left(t, x\left(g_{1}(t)\right), \ldots, x\left(g_{k}(t)\right)\right)\right| \\
& +\frac{1}{t_{1}} \sum_{t=t_{1}}^{\infty} t^{3}\left|f\left(t, x\left(f_{1}(t)\right), \ldots, x\left(f_{k}(t)\right)\right)-d(t)\right| \\
& +\frac{1}{t_{2}} \sum_{t=t_{2}}^{\infty} t^{3}\left|f\left(t, x\left(f_{1}(t)\right), \ldots, x\left(f_{k}(t)\right)\right)-d(t)\right| \\
& <\frac{2 \varepsilon}{16}+\frac{2}{T_{2}} \sum_{t=T_{2}}^{\infty} t H_{t}+\frac{2}{T_{2}} \sum_{t=T_{2}}^{\infty} t^{2} G_{t} \\
& +\frac{2}{T_{2}} \sum_{t=T_{2}}^{\infty} t^{3}\left[F_{t}+|d(t)|\right]<\varepsilon,
\end{aligned}
$$

which means that $S_{L}\left(\Omega\left(A_{*}, B^{*}, T\right)\right)$ is uniformly Cauchy, which together with (87) and Lemma 1 yields that $S_{L}\left(\Omega\left(A_{*}\right.\right.$, $\left.B^{*}, T\right)$ ) is relatively compact. It follows from Lemma 3 that the mapping $S_{L}$ has a fixed point $x=\{x(n)\}_{n \in \mathbb{N}_{\beta}} \in \Omega\left(A_{*}, B^{*}, T\right)$; that is,

$$
\begin{aligned}
x(n) & n L+\sum_{i=1}^{\infty} c(n+i \tau) \\
& -\sum_{i=1}^{\infty} \sum_{t=n+i \tau}^{\infty} h\left(t, x\left(h_{1}(t)\right), \ldots, x\left(h_{k}(t)\right)\right)
\end{aligned}
$$

$$
\begin{aligned}
& +\sum_{i=1}^{\infty} \sum_{s=n+i \tau}^{\infty} \sum_{t=s}^{\infty} g\left(t, x\left(g_{1}(t)\right), \ldots, x\left(g_{k}(t)\right)\right) \\
& -\sum_{p=1}^{\infty} \sum_{i=n+p \tau}^{\infty} \sum_{s=i}^{\infty} \sum_{t=s}^{\infty}\left[f\left(t, x\left(f_{1}(t)\right), \ldots, x\left(f_{k}(t)\right)\right)\right.
\end{aligned}
$$$$
-d(t)], \quad n \geq T,
$$

which gives that

$$
\begin{aligned}
& x(n)-x(n-\tau) \\
& =\tau L-c(n)+\sum_{t=n}^{\infty} h\left(t, x\left(h_{1}(t)\right), \ldots, x\left(h_{k}(t)\right)\right) \\
& \quad-\sum_{s=n}^{\infty} \sum_{t=s}^{\infty} g\left(t, x\left(g_{1}(t)\right), \ldots, x\left(g_{k}(t)\right)\right) \\
& +\sum_{i=n}^{\infty} \sum_{s=i}^{\infty} \sum_{t=s}^{\infty}\left[f\left(t, x\left(f_{1}(t)\right), \ldots, x\left(f_{k}(t)\right)\right)-d(t)\right], \\
& n \geq T+\tau .
\end{aligned}
$$

It is easy to verify that (95) implies that

$$
\begin{aligned}
\Delta & (x(n)-x(n-\tau)+c(n)) \\
= & -h\left(n, x\left(h_{1}(n)\right), \ldots, x\left(h_{k}(n)\right)\right) \\
& +\sum_{t=n}^{\infty} g\left(t, x\left(g_{1}(t)\right), \ldots, x\left(g_{k}(t)\right)\right) \\
& \quad-\sum_{s=n}^{\infty} \sum_{t=s}^{\infty}\left[f\left(t, x\left(f_{1}(t)\right), \ldots, x\left(f_{k}(t)\right)\right)-d(t)\right],
\end{aligned}
$$$$
n \geq T+\tau,
$$

$$
\begin{aligned}
& \Delta^{2}(x(n)-x(n-\tau)+c(n)) \\
& =-\Delta h\left(n, x\left(h_{1}(n)\right), \ldots, x\left(h_{k}(n)\right)\right) \\
& \quad-g\left(n, x\left(g_{1}(n)\right), \ldots, x\left(g_{k}(n)\right)\right) \\
& \quad+\sum_{t=n}^{\infty}\left[f\left(t, x\left(f_{1}(t)\right), \ldots, x\left(f_{k}(t)\right)\right)-d(t)\right], \\
& n \geq T+\tau,
\end{aligned}
$$


which yields that

$$
\begin{aligned}
\Delta^{3}( & x(n)-x(n-\tau)+c(n)) \\
= & -\Delta^{2} h\left(n, x\left(h_{1}(n)\right), \ldots, x\left(h_{k}(n)\right)\right) \\
& -\Delta g\left(n, x\left(g_{1}(n)\right), \ldots, x\left(g_{k}(n)\right)\right) \\
& -\left[f\left(n, x\left(f_{1}(n)\right), \ldots, x\left(f_{k}(n)\right)\right)-d(n)\right], \\
n & \geq T+\tau,
\end{aligned}
$$

which together with (81) gives that $x=\{x(n)\}_{n \in \mathbb{N}_{\beta}} \in \Omega\left(A_{*}\right.$, $\left.B^{*}, T\right)$ is a positive solution of (4). It follows from (78), (79), (95), and Lemma 4 that

$$
\begin{aligned}
& \frac{|x(n)-x(n-\tau)+c(n)|}{n} \\
& =\mid \frac{\tau L}{n}+\frac{1}{n} \sum_{t=n}^{\infty} h\left(t, x\left(h_{1}(t)\right), \ldots, x\left(h_{k}(t)\right)\right) \\
& \quad-\frac{1}{n} \sum_{s=n}^{\infty} \sum_{t=s}^{\infty} g\left(t, x\left(g_{1}(t)\right), \ldots, x\left(g_{k}(t)\right)\right) \\
& \quad+\frac{1}{n} \sum_{i=n}^{\infty} \sum_{s=i}^{\infty} \sum_{t=s}^{\infty}\left[f\left(t, x\left(f_{1}(t)\right), \ldots, x\left(f_{k}(t)\right)\right)\right. \\
& \leq \frac{\tau L}{n}+\frac{1}{n} \sum_{t=n}^{\infty} H_{t}+\frac{1}{n} \sum_{s=n}^{\infty} \sum_{t=s}^{\infty} G_{t} \\
& \quad+\frac{1}{n} \sum_{i=n}^{\infty} \sum_{s=i}^{\infty} \sum_{t=s}^{\infty}\left[H_{t}+|d(t)|\right] \\
& \quad+\frac{1}{n} \sum_{t=n}^{\infty} H_{t}+\frac{1}{n} \sum_{t=n}^{\infty} t G_{t} \\
& \quad-d(t)] \mid
\end{aligned}
$$

that is, (82) holds. The proof of (19) is similar to that of Theorem 5 and is omitted.

(ii) Let $L_{1}, L_{2} \in(A, B)$ and $L_{1} \neq L_{2}$. Similarly we conclude that for each $l \in\{1,2\}$, there exist a constant $T_{l} \geq$ $n_{1}+\tau+|\alpha|$ and a mapping $S_{L_{l}}: \Omega\left(A_{*}, B^{*}, T_{l}\right) \rightarrow l_{\beta}^{\infty}$ satisfying (83)-(87), where $T, L$, and $S_{L}$ are replaced by $T_{l}, L_{l}$, and $S_{L_{l}}$, respectively, and $S_{L_{l}}$ possesses a fixed point $x_{l}=\left\{x_{l}(n)\right\}_{n \in \mathbb{N}_{\beta}} \epsilon$ $\Omega\left(A_{*}, B^{*}, T_{l}\right)$, which is a positive solution of (4); that is,

$$
\begin{aligned}
& x_{l}(n) \\
& =n L_{l}+\sum_{i=1}^{\infty} c(n+i \tau) \\
& \quad-\sum_{i=1}^{\infty} \sum_{t=n+i \tau}^{\infty} h\left(t, x_{l}\left(h_{1}(t)\right), \ldots, x_{l}\left(h_{k}(t)\right)\right) \\
& +\sum_{i=1}^{\infty} \sum_{s=n+i \tau}^{\infty} \sum_{t=s}^{\infty} g\left(t, x_{l}\left(g_{1}(t)\right), \ldots, x_{l}\left(g_{k}(t)\right)\right) \\
& \quad-\sum_{p=1}^{\infty} \sum_{i=n+p \tau}^{\infty} \sum_{s=i}^{\infty} \sum_{t=s}^{\infty}\left[f\left(t, x_{l}\left(f_{1}(t)\right), \ldots, x_{l}\left(f_{k}(t)\right)\right)\right. \\
&
\end{aligned}
$$

Note that (79) and (80) imply that there exists $T_{*}>\max \left\{T_{1}\right.$, $T_{2}$ \} satisfying

$$
\begin{gathered}
\frac{1}{T_{*}}\left(\sum_{t=T_{*}}^{\infty} t H_{t}+\sum_{t=T_{*}}^{\infty} t^{2} G_{t}+\sum_{t=T_{*}}^{\infty} t^{3} F_{t}\right) \\
<\frac{\left|L_{1}-L_{2}\right|}{4} .
\end{gathered}
$$

In view of (15), (99), (100), and Lemma 4, we infer that for any $n \geq T_{*}$

$$
\begin{aligned}
& \left|\frac{x_{1}(n)}{n}-\frac{x_{2}(n)}{n}\right| \\
& =\mid L_{1}-L_{2} \\
& \quad-\frac{1}{n} \sum_{i=1}^{\infty} \sum_{t=n+i \tau}^{\infty}\left[h\left(t, x_{1}\left(h_{1}(t)\right), \ldots, x_{1}\left(h_{k}(t)\right)\right)\right. \\
& +\frac{1}{n} \sum_{i=1}^{\infty} \sum_{s=n+i \tau}^{\infty} \sum_{t=s}^{\infty}\left[g\left(t, x_{1}\left(g_{1}(t)\right), \ldots, x_{1}\left(g_{k}(t)\right)\right)\right. \\
& \quad-\frac{1}{n} \sum_{p=1}^{\infty} \sum_{i=n+p \tau}^{\infty} \sum_{s=i}^{\infty} \sum_{t=s}^{\infty}\left[f\left(t, x_{2}\left(h_{1}(t)\right), \ldots, x_{2}\left(h_{k}(t)\right)\right)\right] \\
& \left.-g\left(t, x_{2}\left(g_{1}(t)\right), \ldots, x_{2}\left(g_{k}(t)\right)\right)\right] \\
& \left.-f\left(t, x_{2}\left(f_{1}(t)\right), \ldots, x_{2}\left(f_{k}(t)\right)\right)\right] \mid
\end{aligned}
$$




$$
\begin{aligned}
& \geq\left|L_{1}-L_{2}\right| \\
& -\frac{1}{T_{*}} \sum_{i=1}^{\infty} \sum_{t=T_{*}+i \tau}^{\infty} \mid h\left(t, x_{1}\left(h_{1}(t)\right), \ldots, x_{1}\left(h_{k}(t)\right)\right) \\
& -h\left(t, x_{2}\left(h_{1}(t)\right), \ldots, x_{2}\left(h_{k}(t)\right)\right) \\
& -\frac{1}{T_{*}} \sum_{i=1}^{\infty} \sum_{s=T_{*}+i \tau}^{\infty} \sum_{t=s}^{\infty} \mid g\left(t, x_{1}\left(g_{1}(t)\right), \ldots, x_{1}\left(g_{k}(t)\right)\right) \\
& -g\left(t, x_{2}\left(g_{1}(t)\right), \ldots, x_{2}\left(g_{k}(t)\right)\right) \\
& -\frac{1}{T_{*}} \sum_{p=1}^{\infty} \sum_{i=T_{*}+p \tau}^{\infty} \sum_{s=i}^{\infty} \sum_{t=s}^{\infty} \mid f\left(t, x_{1}\left(f_{1}(t)\right), \ldots, x_{1}\left(f_{k}(t)\right)\right) \\
& -f\left(t, x_{2}\left(f_{1}(t)\right), x_{2}\left(f_{2}(t)\right), \ldots,\right. \\
& \left.x_{2}\left(f_{k}(t)\right)\right) \mid \\
& \geq\left|L_{1}-L_{2}\right| \\
& -\frac{2}{T_{*}}\left(\sum_{t=T_{*}}^{\infty} t H_{t}+\sum_{t=T_{*}}^{\infty} t^{2} G_{t}+\sum_{t=T_{*}}^{\infty} t^{3} F_{t}\right) \\
& >\frac{\left|L_{1}-L_{2}\right|}{2}>0 \text {, }
\end{aligned}
$$

which yields that $x_{1} \neq x_{2}$. Thus (4) possesses uncountably many positive solutions in $\Omega\left(A_{*}, B^{*}, T\right)$. This completes the proof.

Theorem 10. Assume that there exist three nonnegative sequences $\left\{F_{n}\right\}_{n \in \mathbb{N}_{n_{0}}},\left\{G_{n}\right\}_{n \in \mathbb{N}_{n_{0}}}$, and $\left\{H_{n}\right\}_{n \in \mathbb{N}_{n_{0}}}$ satisfying (15)-(17), (80), and

$$
b(n)=1, \quad n \in \mathbb{N}_{n_{0}} .
$$

Then

(i) equation (4) possesses uncountably many positive solutions $x=\{x(n)\}_{n \in \mathbb{N}_{\beta}} \in l_{\beta}^{\infty}$ with (19) and

$$
\lim _{n \rightarrow \infty} \frac{x(n)+x(n-\tau)+c(n)}{n} \in(2 A, 2 B) ;
$$

(ii) equation (4) possesses uncountably many positive solutions in $l_{\beta}^{\infty}$.

Proof. (i) Let $L \in(A, B)$. It follows from (15)-(17) and (80) that there exists $T \geq n_{0}+\tau+\alpha$ satisfying (83) and

$$
\begin{aligned}
\frac{1}{T} \sum_{t=T}^{\infty} H_{t} & +\frac{1}{T} \sum_{s=T}^{\infty} \sum_{t=s}^{\infty} G_{t} \\
& +\frac{1}{T} \sum_{i=T}^{\infty} \sum_{s=i}^{\infty} \sum_{t=s}^{\infty}\left[F_{t}+|d(t)|\right] \\
< & \frac{1}{2} \min \{L-A, B-L\} .
\end{aligned}
$$

Define a mapping $S_{L}: \Omega\left(A_{*}, B^{*}, T\right) \rightarrow l_{\beta}^{\infty}$ by

$$
\begin{aligned}
& \left(S_{L} x\right)(n) \\
& =\left\{\begin{array}{l}
n L+\sum_{i=1}^{\infty}(-1)^{i} c(n+i \tau) \\
+\sum_{s=1}^{\infty} \sum_{t=n+(2 s-1) \tau}^{n+2 s \tau-1} h\left(t, x\left(h_{1}(t)\right), \ldots, x\left(h_{k}(t)\right)\right) \\
-\sum_{i=1}^{\infty} \sum_{s=n+(2 i-1) \tau}^{n+2 i \tau-1} \sum_{t=s}^{\infty} g\left(t, x\left(g_{1}(t)\right), \ldots, x\left(g_{k}(t)\right)\right) \\
+\sum_{p=1}^{\infty} \sum_{i=n+(2 p-1) \tau}^{n+2 p \tau-1} \sum_{s=i}^{\infty} \sum_{t=s}^{\infty}\left[f\left(t, x\left(f_{1}(t)\right), \ldots, x\left(f_{k}(t)\right)\right)\right. \\
\left.\frac{n}{T}\left(S_{L} x\right)(T), \quad-d(t)\right], \quad n \geq T, \\
\end{array}\right.
\end{aligned}
$$

for any $x=\{x(n)\}_{n \in \mathbb{N}_{\beta}} \in \Omega\left(A_{*}, B^{*}, T\right)$.

Now we show that (86) and (87) hold. It follows from (15), (83), (104), and (105) that for any $x=\{x(n)\}_{n \in \mathbb{N}_{\beta}} \in \Omega\left(A_{*}\right.$, $\left.B^{*}, T\right)$

$$
\begin{aligned}
\mid & \frac{\left(S_{L} x\right)(n)}{n}-L \mid \\
= & \mid \frac{1}{n} \sum_{i=1}^{\infty}(-1)^{i} c(n+i \tau) \\
& +\frac{1}{n} \sum_{s=1}^{\infty} \sum_{t=n+(2 s-1) \tau}^{n+2 s \tau-1} h\left(t, x\left(h_{1}(t)\right), \ldots, x\left(h_{k}(t)\right)\right) \\
& -\frac{1}{n} \sum_{i=1}^{\infty} \sum_{s=n+(2 i-1) \tau} \sum_{t=s}^{\infty} g\left(t, x\left(g_{1}(t)\right), \ldots, x\left(g_{k}(t)\right)\right) \\
& +\frac{1}{n} \sum_{p=1}^{\infty} \sum_{i=n+(2 p-1) \tau}^{n+2 p \tau-1} \sum_{s=i}^{\infty} \sum_{t=s}^{\infty}\left[f\left(t, x\left(f_{1}(t)\right), \ldots, x\left(f_{k}(t)\right)\right)\right. \\
\leq & \frac{1}{n} \sum_{i=1}^{\infty}|c(n+i \tau)| \\
& +\frac{1}{n} \sum_{s=1}^{\infty} \sum_{t=n+(2 s-1) \tau}^{n+2 s \tau-1}\left|h\left(t, x\left(h_{1}(t)\right), \ldots, x\left(h_{k}(t)\right)\right)\right| \\
& \sum_{i=1}^{\infty} \sum_{s=n+(2 i-1) \tau} \sum_{t=s}^{\infty}\left|g\left(t, x\left(g_{1}(t)\right), \ldots, x\left(g_{k}(t)\right)\right)\right|
\end{aligned}
$$




$$
\begin{aligned}
& +\frac{1}{n} \sum_{p=1}^{\infty} \sum_{i=n+(2 p-1) \tau}^{n+2 p \tau-1} \sum_{s=i}^{\infty} \sum_{t=s}^{\infty}\left[\mid f\left(t, x\left(f_{1}(t)\right), \ldots\right.\right. \\
& \left.\left.x\left(f_{k}(t)\right)\right)|+| d(t) \mid\right] \\
& <\frac{1}{2} \min \{L-A, B-L\} \\
& +\frac{1}{T} \sum_{t=T}^{\infty}\left|h\left(t, x\left(h_{1}(t)\right), \ldots, x\left(h_{k}(t)\right)\right)\right| \\
& +\frac{1}{T} \sum_{s=T}^{\infty} \sum_{t=s}^{\infty}\left|g\left(t, x\left(g_{1}(t)\right), \ldots, x\left(g_{k}(t)\right)\right)\right| \\
& +\frac{1}{T} \sum_{i=T}^{\infty} \sum_{s=i}^{\infty} \sum_{t=s}^{\infty}\left[\left|f\left(t, x\left(f_{1}(t)\right), \ldots, x\left(f_{k}(t)\right)\right)\right|\right. \\
& +|d(t)|] \\
& \leq \frac{1}{2} \min \{L-A, B-L\} \\
& +\frac{1}{T} \sum_{t=T}^{\infty} H_{t}+\frac{1}{T} \sum_{s=T}^{\infty} \sum_{t=s}^{\infty} G_{t} \\
& +\frac{1}{T} \sum_{i=T}^{\infty} \sum_{s=i}^{\infty} \sum_{t=s}^{\infty}\left[F_{t}+|d(t)|\right] \\
& <\min \{L-A, B-L\}, \quad n \geq T, \\
& \left|\frac{\left(S_{L} x\right)(n)}{n}-L\right|=\left|\frac{n}{T} \cdot \frac{\left(S_{L} x\right)(T)}{n}-L\right| \\
& <\min \{L-A, B-L\}, \quad \beta \leq n<T, \\
& \left|\frac{\left(S_{L} x\right)(n)}{n}\right| \\
& =\mid L+\frac{1}{n} \sum_{i=1}^{\infty}(-1)^{i} c(n+i \tau) \\
& +\frac{1}{n} \sum_{s=1}^{\infty} \sum_{t=n+(2 s-1) \tau}^{n+2 s \tau-1} h\left(t, x\left(h_{1}(t)\right), \ldots, x\left(h_{k}(t)\right)\right) \\
& -\frac{1}{n} \sum_{i=1}^{\infty} \sum_{s=n+(2 i-1) \tau}^{n+2 i \tau-1} \sum_{t=s}^{\infty} g\left(t, x\left(g_{1}(t)\right), \ldots, x\left(g_{k}(t)\right)\right) \\
& +\frac{1}{n} \sum_{p=1}^{\infty} \sum_{i=n+(2 p-1)}^{n+2 p \tau-1} \sum_{s=i}^{\infty} \sum_{t=s}^{\infty}\left[f\left(t, x\left(f_{1}(t)\right), \ldots, x\left(f_{k}(t)\right)\right)\right. \\
& -d(t)]
\end{aligned}
$$

$$
\begin{aligned}
& \leq L+\frac{1}{n} \sum_{i=1}^{\infty}|c(n+i \tau)| \\
& +\frac{1}{n} \sum_{s=1}^{\infty} \sum_{t=n+(2 s-1) \tau}^{n+2 s \tau-1}\left|h\left(t, x\left(h_{1}(t)\right), \ldots, x\left(h_{k}(t)\right)\right)\right| \\
& +\frac{1}{n} \sum_{i=1}^{\infty} \sum_{s=n+(2 i-1) \tau}^{n+2 i \tau-1} \sum_{t=s}^{\infty}\left|g\left(t, x\left(g_{1}(t)\right), \ldots, x\left(g_{k}(t)\right)\right)\right| \\
& +\frac{1}{n} \sum_{p=1}^{\infty} \sum_{i=n+(2 p-1) \tau}^{n+2 p \tau-1} \sum_{s=i}^{\infty} \sum_{t=s}^{\infty}\left[\mid f\left(t, x\left(f_{1}(t)\right), \ldots\right.\right. \\
& \left.\left.x\left(f_{k}(t)\right)\right)|+| d(t) \mid\right] \\
& <L+\frac{1}{2} \min \{L-A, B-L\} \\
& +\frac{1}{T} \sum_{t=T}^{\infty}\left|h\left(t, x\left(h_{1}(t)\right), \ldots, x\left(h_{k}(t)\right)\right)\right| \\
& +\frac{1}{T} \sum_{s=T}^{\infty} \sum_{t=s}^{\infty}\left|g\left(t, x\left(g_{1}(t)\right), \ldots, x\left(g_{k}(t)\right)\right)\right| \\
& +\frac{1}{T} \sum_{i=T}^{\infty} \sum_{s=i}^{\infty} \sum_{t=s}^{\infty}\left[\left|f\left(t, x\left(f_{1}(t)\right), \ldots, x\left(f_{k}(t)\right)\right)\right|\right. \\
& +|d(t)|] \\
& \leq L+\frac{1}{2} \min \{L-A, B-L\}+\frac{1}{T} \sum_{t=T}^{\infty} H_{t} \\
& +\frac{1}{T} \sum_{s=T}^{\infty} \sum_{t=s}^{\infty} G_{t}+\frac{1}{T} \sum_{i=T}^{\infty} \sum_{s=i}^{\infty} \sum_{t=s}^{\infty}\left[F_{t}+|d(t)|\right] \\
& <L+\min \{L-A, B-L\} \leq B, \quad n \geq T, \\
& \left|\frac{\left(S_{L} x\right)(T)}{T}\right|=\frac{n}{T}\left|\frac{\left(S_{L} x\right)(T)}{n}\right| \leq B, \quad \beta \leq n<T,
\end{aligned}
$$

which yield that (86) and (87) hold.

Next we show that $S_{L}$ is continuous and $S_{L}\left(\Omega\left(A_{*}, B^{*}, T\right)\right)$ is uniformly Cauchy. Let $x^{w}=\left\{x^{w}(n)\right\}_{n \in \mathbb{N}_{\beta}}$ and $x=$ $\{x(n)\}_{n \in \mathbb{N}_{\beta}} \in \Omega\left(A_{*}, B^{*}, T\right)$ with (90). Using (16), (17), (80), and the continuity of $f, g$, and $h$, we know that for given $\varepsilon>0$, there exist $T_{1}, T_{2}, T_{3}$, and $T_{4} \in \mathbb{N}$ with $T_{4}>T_{3}>T_{2}>T_{1}>$ $T+\tau$ satisfying

$$
\begin{array}{r}
\frac{1}{T} \max \left\{\sum_{t=T+\tau}^{T_{1}} \mid h\left(t, x^{w}\left(h_{1}(t)\right), \ldots, x^{w}\left(h_{k}(t)\right)\right)\right. \\
-h\left(t, x\left(h_{1}(t)\right), \ldots, x\left(h_{k}(t)\right)\right) \mid,
\end{array}
$$




$$
\begin{aligned}
& \sum_{s=T+\tau}^{T_{1}} \sum_{t=s}^{T_{2}} \mid g\left(t, x^{w}\left(g_{1}(t)\right), \ldots, x^{w}\left(g_{k}(t)\right)\right) \\
& -g\left(t, x\left(g_{1}(t)\right), \ldots, x\left(g_{k}(t)\right)\right) \mid, \\
& \sum_{i=T+\tau}^{T_{1}} \sum_{s=i}^{T_{2}} \sum_{t=s}^{T_{3}} \mid f\left(t, x^{w}\left(f_{1}(t)\right), \ldots, x^{w}\left(f_{k}(t)\right)\right) \\
& \left.-f\left(t, x\left(f_{1}(t)\right), \ldots, x\left(f_{k}(t)\right)\right) \mid\right\} \\
& <\frac{\varepsilon}{16}, \quad w \geq T_{4}, \\
& \frac{1}{T} \max \left\{\sum_{t=T_{1}+1}^{\infty} H_{t}+\sum_{s=T_{1}+1}^{\infty} \sum_{t=s}^{\infty} G_{t}\right. \\
& +\sum_{i=T_{1}+1}^{\infty} \sum_{s=i}^{\infty} \sum_{t=s}^{\infty} F_{t} \\
& \sum_{s=T+\tau}^{T_{1}} \sum_{t=T_{2}+1}^{\infty} G_{t}+\sum_{i=T+\tau}^{T_{1}} \sum_{s=i}^{T_{2}} \sum_{t=T_{3}+1}^{\infty} F_{t} \\
& \left.+\sum_{i=T+\tau}^{T_{1}} \sum_{s=T_{2}+1}^{\infty} \sum_{t=s}^{\infty} F_{t}\right\}<\frac{\varepsilon}{16}, \\
& \frac{1}{n} \sum_{i=1}^{\infty}|c(n+i \tau)|<\frac{\varepsilon}{16}, \quad n \geq T_{4} .
\end{aligned}
$$

Combining (15) and (105)-(108), we infer that

$$
\begin{aligned}
& \left\|S_{L} x^{w}-S_{L} x\right\| \\
& \leq \frac{1}{n} \sum_{s=1}^{\infty} \sum_{t=n+(2 s-1) \tau}^{n+2 s \tau-1} \mid h\left(t, x^{w}\left(h_{1}(t)\right), \ldots, x^{w}\left(h_{k}(t)\right)\right) \\
& -h\left(t, x\left(h_{1}(t)\right), \ldots, x\left(h_{k}(t)\right)\right) \mid \\
& +\frac{1}{n} \sum_{i=1}^{\infty} \sum_{s=n+(2 i-1) \tau} \sum_{t=s}^{n+2 i \tau-1} \mid g\left(t, x^{w}\left(g_{1}(t)\right), \ldots, x^{w}\left(g_{k}(t)\right)\right) \\
& +\frac{1}{n} \sum_{p=1}^{\infty} \sum_{i=n+(2 p-1) \tau} \sum_{s=i}^{n+2 p \tau-1} \sum_{t=s}^{\infty} \mid f\left(t, x^{w}\left(f_{1}(t)\right), \ldots, x^{w}\left(f_{k}(t)\right)\right) \\
& -f\left(t, x\left(f_{1}(t)\right), \ldots, x\left(f_{k}(t)\right)\right) \mid
\end{aligned}
$$

$$
\begin{array}{r}
\leq \frac{1}{T} \sum_{t=T+\tau}^{T_{1}} \mid h\left(t, x^{w}\left(h_{1}(t)\right), \ldots, x^{w}\left(h_{k}(t)\right)\right) \\
-h\left(t, x\left(h_{1}(t)\right), \ldots, x\left(h_{k}(t)\right)\right) \mid \\
+\frac{1}{T} \sum_{t=T_{1}+1}^{\infty} \mid h\left(t, x^{w}\left(h_{1}(t)\right), \ldots, x^{w}\left(h_{k}(t)\right)\right)
\end{array}
$$$$
-h\left(t, x\left(h_{1}(t)\right), \ldots, x\left(h_{k}(t)\right)\right)
$$$$
+\frac{1}{T} \sum_{s=T+\tau}^{T_{1}} \sum_{t=s}^{T_{2}} \mid g\left(t, x^{w}\left(g_{1}(t)\right), \ldots, x^{w}\left(g_{k}(t)\right)\right)
$$$$
-g\left(t, x\left(g_{1}(t)\right), \ldots, x\left(g_{k}(t)\right)\right) \mid
$$$$
+\frac{1}{T} \sum_{s=T+\tau}^{T_{1}} \sum_{t=T_{2}+1}^{\infty} \mid g\left(t, x^{w}\left(g_{1}(t)\right), \ldots, x^{w}\left(g_{k}(t)\right)\right)
$$$$
-g\left(t, x\left(g_{1}(t)\right), \ldots, x\left(g_{k}(t)\right)\right)
$$$$
+\frac{1}{T} \sum_{s=T_{1}+1}^{\infty} \sum_{t=s}^{\infty} \mid g\left(t, x^{w}\left(g_{1}(t)\right), \ldots, x^{w}\left(g_{k}(t)\right)\right)
$$$$
-g\left(t, x\left(g_{1}(t)\right), \ldots, x\left(g_{k}(t)\right)\right)
$$$$
+\frac{1}{T} \sum_{i=T+\tau}^{T_{1}} \sum_{s=i}^{T_{2}} \sum_{t=s}^{T_{3}} \mid f\left(t, x^{w}\left(f_{1}(t)\right), \ldots, x^{w}\left(f_{k}(t)\right)\right)
$$$$
-f\left(t, x\left(f_{1}(t)\right), \ldots, x\left(f_{k}(t)\right)\right) \mid
$$$$
+\frac{1}{T} \sum_{i=T+\tau}^{T_{1}} \sum_{s=i}^{T_{2}} \sum_{t=T_{3}+1}^{\infty} \mid f\left(t, x^{w}\left(f_{1}(t)\right), \ldots, x^{w}\left(f_{k}(t)\right)\right)
$$$$
-f\left(t, x\left(f_{1}(t)\right), \ldots, x\left(f_{k}(t)\right)\right)
$$$$
+\frac{1}{T} \sum_{i=T+\tau}^{T_{1}} \sum_{s=T_{2}+1}^{\infty} \sum_{t=s}^{\infty} \mid f\left(t, x^{w}\left(f_{1}(t)\right), \ldots, x^{w}\left(f_{k}(t)\right)\right)
$$$$
-f\left(t, x\left(f_{1}(t)\right), \ldots, x\left(f_{k}(t)\right)\right)
$$$$
+\frac{1}{T} \sum_{i=T_{1}+1}^{\infty} \sum_{s=i}^{\infty} \sum_{t=s}^{\infty} \mid f\left(t, x^{w}\left(f_{1}(t)\right), \ldots, x^{w}\left(f_{k}(t)\right)\right)
$$$$
-f\left(t, x\left(f_{1}(t)\right), \ldots, x\left(f_{k}(t)\right)\right) \mid
$$$$
<\frac{\varepsilon}{16}+\frac{2}{T} \sum_{t=T_{1}+1}^{\infty} H_{t}+\frac{\varepsilon}{16}+\frac{2}{T} \sum_{s=T+\tau}^{T_{1}} \sum_{t=T_{2}+1}^{\infty} G_{t}
$$$$
+\frac{2}{T} \sum_{s=T_{1}+1}^{\infty} \sum_{t=s}^{\infty} G_{t}+\frac{\varepsilon}{16}+\frac{2}{T} \sum_{i=T+\tau}^{T_{1}} \sum_{s=i}^{T_{2}} \sum_{t=T_{3}+1}^{\infty} F_{t}
$$ 


$$
\begin{aligned}
& +\frac{2}{T} \sum_{i=T+\tau}^{T_{1}} \sum_{s=T_{2}+1}^{\infty} \sum_{t=s}^{\infty} F_{t} \\
& +\frac{2}{T} \sum_{i=T_{1}+1}^{\infty} \sum_{s=i}^{\infty} \sum_{t=s}^{\infty} F_{t}<\varepsilon, \quad w \geq T_{4},
\end{aligned}
$$

which implies that $S_{L}$ is continuous in $\Omega\left(A_{*}, B^{*}, T\right)$. It follows from (15), (108), and (109) that for any $x=\{x(n)\}_{n \in \mathbb{N}_{\beta}} \in$ $\Omega\left(A_{*}, B^{*}, T\right)$ and $t_{1}, t_{2} \geq T_{4}$

$$
\begin{aligned}
& \left|\frac{\left(S_{L} x\right)\left(t_{1}\right)}{t_{1}}-\frac{\left(S_{L} x\right)\left(t_{2}\right)}{t_{2}}\right| \\
& =\mid \frac{1}{t_{1}} \sum_{i=1}^{\infty}(-1)^{i} c\left(t_{1}+i \tau\right)-\frac{1}{t_{2}} \sum_{i=1}^{\infty}(-1)^{i} c\left(t_{2}+i \tau\right) \\
& +\frac{1}{t_{1}} \sum_{s=1}^{\infty} \sum_{t=t_{1}+(2 s-1) \tau}^{t_{1}+2 s \tau-1} h\left(t, x\left(h_{1}(t)\right), \ldots, x\left(h_{k}(t)\right)\right) \\
& -\frac{1}{t_{2}} \sum_{s=1}^{\infty} \sum_{t=t_{2}+(2 s-1) \tau}^{t_{2}+2 s \tau-1} h\left(t, x\left(h_{1}(t)\right), \ldots, x\left(h_{k}(t)\right)\right) \\
& -\frac{1}{t_{1}} \sum_{i=1}^{\infty} \sum_{s=t_{1}+(2 i-1) \tau}^{t_{1}+2 i \tau-1} \sum_{t=s}^{\infty} g\left(t, x\left(g_{1}(t)\right), \ldots, x\left(g_{k}(t)\right)\right) \\
& +\frac{1}{t_{2}} \sum_{i=1}^{\infty} \sum_{s=t_{2}+(2 i-1) \tau}^{t_{2}+2 i \tau-1} \sum_{t=s}^{\infty} g\left(t, x\left(g_{1}(t)\right), \ldots, x\left(g_{k}(t)\right)\right) \\
& +\frac{1}{t_{1}} \sum_{p=1}^{\infty} \sum_{i=t_{1}+(2 p-1) \tau}^{t_{1}+2 p \tau-1} \sum_{s=i}^{\infty} \sum_{t=s}^{\infty}\left[f\left(t, x\left(f_{1}(t)\right), \ldots, x\left(f_{k}(t)\right)\right)\right. \\
& -d(t)] \\
& -\frac{1}{t_{2}} \sum_{p=1}^{\infty} \sum_{i=t_{2}+(2 p-1)}^{t_{2}+2 p \tau-1} \sum_{\tau=i}^{\infty} \sum_{t=s}^{\infty}\left[f\left(t, x\left(f_{1}(t)\right), \ldots, x\left(f_{k}(t)\right)\right)\right. \\
& -d(t)] \\
& \leq \frac{1}{t_{1}} \sum_{i=1}^{\infty}\left|c\left(t_{1}+i \tau\right)\right| \\
& +\frac{1}{t_{2}} \sum_{i=1}^{\infty}\left|c\left(t_{2}+i \tau\right)\right|+\frac{2}{T_{4}} \sum_{t=T_{4}+\tau}^{\infty} H_{t} \\
& +\frac{2}{T_{4}} \sum_{s=T_{4}+\tau}^{\infty} \sum_{t=s}^{\infty} G_{t} \\
& +\frac{2}{T_{4}} \sum_{i=T_{4}+\tau}^{\infty} \sum_{s=i}^{\infty} \sum_{t=s}^{\infty}\left[F_{t}+|d(t)|\right]<\varepsilon,
\end{aligned}
$$

which means that $S_{L}\left(\Omega\left(A_{*}, B^{*}, T\right)\right)$ is uniformly Cauchy, which together with $(87)$ and Lemma 1 yields that $S_{L}\left(\Omega\left(A_{*}\right.\right.$, $\left.\left.B^{*}, T\right)\right)$ is relatively compact. It follows from Lemma 3 that the mapping $S_{L}$ has a fixed point $x=\{x(n)\}_{n \in \mathbb{N}_{\beta}} \in \Omega\left(A_{*}, B^{*}, T\right)$; that is,

$$
\begin{aligned}
& x(n) \\
& =n L+\sum_{i=1}^{\infty}(-1)^{i} c(n+i \tau) \\
& +\sum_{s=1}^{\infty} \sum_{t=n+(2 s-1) \tau}^{n+2 s \tau-1} h\left(t, x\left(h_{1}(t)\right), \ldots, x\left(h_{k}(t)\right)\right) \\
& -\sum_{i=1}^{\infty} \sum_{s=n+(2 i-1) \tau}^{n+2 i \tau-1} \sum_{t=s}^{\infty} g\left(t, x\left(g_{1}(t)\right), \ldots, x\left(g_{k}(t)\right)\right) \\
& +\sum_{p=1}^{\infty} \sum_{i=n+(2 p-1) \tau}^{n+2 p \tau-1} \sum_{s=i}^{\infty} \sum_{t=s}^{\infty}\left[f\left(t, x\left(f_{1}(t)\right), \ldots, x\left(f_{k}(t)\right)\right)\right. \\
&
\end{aligned}
$$

which gives that

$$
\begin{aligned}
& x(n)+x(n-\tau) \\
& =(2 n-\tau) L-c(n) \\
& +\sum_{t=n}^{\infty} h\left(t, x\left(h_{1}(t)\right), \ldots, x\left(h_{k}(t)\right)\right) \\
& -\sum_{s=n}^{\infty} \sum_{t=s}^{\infty} g\left(t, x\left(g_{1}(t)\right), \ldots, x\left(g_{k}(t)\right)\right) \\
& +\sum_{i=n}^{\infty} \sum_{s=i}^{\infty} \sum_{t=s}^{\infty}\left[f\left(t, x\left(f_{1}(t)\right), \ldots, x\left(f_{k}(t)\right)\right)\right. \\
& -d(t)], \\
& n \geq T+\tau .
\end{aligned}
$$

It follows from (113) that

$$
\begin{aligned}
\Delta & (x(n)+x(n-\tau)+c(n)) \\
= & 2 L-h\left(n, x\left(h_{1}(n)\right), \ldots, x\left(h_{k}(n)\right)\right) \\
& +\sum_{t=n}^{\infty} g\left(t, x\left(g_{1}(t)\right), \ldots, x\left(g_{k}(t)\right)\right) \\
& -\sum_{s=n}^{\infty} \sum_{t=s}^{\infty}\left[f\left(t, x\left(f_{1}(t)\right), \ldots, x\left(f_{k}(t)\right)\right)-d(t)\right],
\end{aligned}
$$$$
n \geq T+\tau \text {, }
$$ 


$$
\begin{aligned}
\Delta^{2} & (x(n)+x(n-\tau)+c(n)) \\
= & -\Delta h\left(n, x\left(h_{1}(n)\right), \ldots, x\left(h_{k}(n)\right)\right) \\
& -g\left(n, x\left(g_{1}(n)\right), \ldots, x\left(g_{k}(n)\right)\right) \\
& +\sum_{t=n}^{\infty}\left[f\left(t, x\left(f_{1}(t)\right), \ldots, x\left(f_{k}(t)\right)\right)-d(t)\right],
\end{aligned}
$$$$
n \geq T+\tau,
$$

which yields that

$$
\begin{aligned}
& \Delta^{3}(x(n)+x(n-\tau)+c(n)) \\
& =-\Delta^{2} h\left(n, x\left(h_{1}(n)\right), \ldots, x\left(h_{k}(n)\right)\right) \\
& -\Delta g\left(n, x\left(g_{1}(n)\right), \ldots, x\left(g_{k}(n)\right)\right) \\
& -\left[f\left(n, x\left(f_{1}(n)\right), \ldots, x\left(f_{k}(n)\right)\right)-d(n)\right], \\
& n \geq T+\tau,
\end{aligned}
$$

which together with (102) means that $x=\{x(n)\}_{n \in \mathbb{N}_{\beta}} \in \Omega\left(A_{*}\right.$, $\left.B^{*}, T\right)$ is a positive solution of (4). In view of (15)-(17) and (113), we get that

$$
\begin{aligned}
\mid & \frac{x(n)+x(n-\tau)+c(n)}{n}-2 L \mid \\
=\mid & -\frac{\tau L}{n}+\frac{1}{n} \sum_{t=n}^{\infty} h\left(t, x\left(h_{1}(t)\right), \ldots, x\left(h_{k}(t)\right)\right) \\
& -\frac{1}{n} \sum_{s=n}^{\infty} \sum_{t=s}^{\infty} g\left(t, x\left(g_{1}(t)\right), \ldots, x\left(g_{k}(t)\right)\right) \\
& +\frac{1}{n} \sum_{i=n}^{\infty} \sum_{s=i}^{\infty} \sum_{t=s}^{\infty}\left[f\left(t, x\left(f_{1}(t)\right), \ldots, x\left(f_{k}(t)\right)\right)\right. \\
\leq & \frac{\tau L}{n}+\frac{1}{n} \sum_{t=n}^{\infty} H_{t}+\frac{1}{n} \sum_{s=n}^{\infty} \sum_{t=s}^{\infty} G_{t} \\
& +\frac{1}{n} \sum_{i=n}^{\infty} \sum_{s=i}^{\infty} \sum_{t=s}^{\infty}\left[F_{t}+|d(t)|\right] \longrightarrow 0 \quad \text { as } n \longrightarrow \infty ;
\end{aligned}
$$

that is, (103) holds. Similar to the proof of Theorem 5, we deduce that (19) holds.

(ii) Let $L_{1}, L_{2} \in(A, B)$ and $L_{1} \neq L_{2}$. Similarly we obtain that for each $l \in\{1,2\}$, there exist a constant $T_{l} \geq n_{0}+\tau+\alpha$ and two mappings $S_{L_{l}}: \Omega\left(A_{*}, B^{*}, T_{l}\right) \rightarrow l_{\beta}^{\infty}$ satisfying (83), (104), and (105), where $T, L$, and $S_{L}$ are replaced by $T_{l}, L_{l}$, and $S_{L_{l}}$, respectively, and $S_{L_{l}}$ possesses a fixed point $x_{l}=$ $\left\{x_{l}(n)\right\}_{n \in \mathbb{N}_{\beta}} \in \Omega\left(A_{*}, B^{*}, T_{l}\right)$, which is a positive solution of (4); that is,

$$
\begin{aligned}
& x_{l}(n) \\
& =n L_{l}+\sum_{i=1}^{\infty}(-1)^{i} c(n+i \tau)
\end{aligned}
$$

$$
\begin{gathered}
+\sum_{i=1}^{\infty} \sum_{t=n+(2 s-1) \tau}^{n+2 s \tau-1} h\left(t, x_{l}\left(h_{1}(t)\right), \ldots, x_{l}\left(h_{k}(t)\right)\right) \\
-\sum_{i=1}^{\infty} \sum_{s=n+(2 i-1) \tau}^{n+2 i \tau-1} \sum_{t=s}^{\infty} g\left(t, x_{l}\left(g_{1}(t)\right), \ldots, x_{l}\left(g_{k}(t)\right)\right) \\
+\sum_{p=1}^{\infty} \sum_{i=n+(2 p-1) \tau}^{n+2 p \tau-1} \sum_{s=i}^{\infty} \sum_{t=s}^{\infty}\left[f\left(t, x_{l}\left(f_{1}(t)\right), \ldots, x_{l}\left(f_{k}(t)\right)\right)\right. \\
-d(t)], \quad n \geq T_{l} .
\end{gathered}
$$

Note that (16) and (17) imply that there exists $T_{*}>\max \left\{T_{1}\right.$, $\left.T_{2}\right\}$ satisfying

$$
\begin{gathered}
\frac{1}{T_{*}}\left(\sum_{t=T_{*}+\tau}^{\infty} H_{t}+\sum_{s=T_{*}+\tau}^{\infty} \sum_{t=s}^{\infty} G_{t}+\sum_{i=T_{*}+\tau}^{\infty} \sum_{s=i}^{\infty} \sum_{t=s}^{\infty} F_{t}\right) \\
<\frac{\left|L_{1}-L_{2}\right|}{4} .
\end{gathered}
$$

In view of (15), (117), and (118), we infer that for any $n \geq T_{*}$

$$
\begin{aligned}
& \quad\left|\frac{x_{1}(n)}{n}-\frac{x_{2}(n)}{n}\right| \\
& =\mid L_{1}-L_{2} \\
& \quad+\frac{1}{n} \sum_{s=1}^{\infty} \sum_{t=n+(2 s-1) \tau}^{n+2 s \tau-1}\left[h\left(t, x_{1}\left(h_{1}(t)\right), \ldots, x_{1}\left(h_{k}(t)\right)\right)\right. \\
& \quad-\frac{1}{n} \sum_{i=1}^{\infty} \sum_{s=n+(2 i-1) \tau}^{n+2 i \tau-1} \sum_{t=s}^{\infty}\left[g\left(t, x_{1}\left(g_{1}(t)\right), \ldots, x_{1}\left(g_{k}(t)\right)\right)\right. \\
& \left.\quad-g\left(t, x_{2}\left(g_{1}(t)\right), \ldots, x_{2}\left(g_{k}(t)\right)\right)\right]
\end{aligned}
$$




$$
\begin{aligned}
& +\frac{1}{n} \sum_{p=1}^{\infty} \sum_{i=n+(2 p-1) \tau}^{n+2 p \tau-1} \sum_{s=i}^{\infty} \sum_{t=s}^{\infty}\left[f \left(t, x_{1}\left(f_{1}(t)\right),\right.\right. \\
& x_{1}\left(f_{2}(t)\right), \ldots, \\
& \left.x_{1}\left(f_{k}(t)\right)\right) \\
& -f\left(t, x_{2}\left(f_{1}(t)\right), \ldots,\right. \\
& \left.\left.x_{2}\left(f_{k}(t)\right)\right)\right] \\
& \geq\left|L_{1}-L_{2}\right| \\
& -\frac{1}{T_{*}} \sum_{t=T_{*}+\tau}^{\infty} \mid h\left(t, x_{1}\left(h_{1}(t)\right), \ldots, x_{1}\left(h_{k}(t)\right)\right) \\
& -h\left(t, x_{2}\left(h_{1}(t)\right), \ldots, x_{2}\left(h_{k}(t)\right)\right) \mid \\
& -\frac{1}{T_{*}} \sum_{s=T_{*}+\tau}^{\infty} \sum_{t=s}^{\infty} \mid g\left(t, x_{1}\left(g_{1}(t)\right), \ldots, x_{1}\left(g_{k}(t)\right)\right) \\
& -g\left(t, x_{2}\left(g_{1}(t)\right), \ldots, x_{2}\left(g_{k}(t)\right)\right) \\
& -\frac{1}{T_{*}} \sum_{i=T_{*}+\tau}^{\infty} \sum_{s=i}^{\infty} \sum_{t=s}^{\infty} \mid f\left(t, x_{1}\left(f_{1}(t)\right), \ldots, x_{1}\left(f_{k}(t)\right)\right) \\
& -f\left(t, x_{2}\left(f_{1}(t)\right), \ldots, x_{2}\left(f_{k}(t)\right)\right) \\
& \geq\left|L_{1}-L_{2}\right| \\
& -\frac{2}{T_{*}}\left(\sum_{t=T_{*}+\tau}^{\infty} H_{t}+\sum_{s=T_{*}+\tau}^{\infty} \sum_{t=s}^{\infty} G_{t}+\sum_{i=T_{*}+\tau}^{\infty} \sum_{s=i}^{\infty} \sum_{t=s}^{\infty} F_{t}\right) \\
& >\frac{\left|L_{1}-L_{2}\right|}{2}>0
\end{aligned}
$$

which yields that $x_{1} \neq x_{2}$. Therefore (4) possesses uncountably many positive solutions in $l_{\beta}^{\infty}$. This completes the proof.

\section{Illustrative Examples}

Now we suggest six examples to explain the results presented in Section 2. Notice that none of the known results can be applied to these examples.

Example 1. Consider the third order nonlinear neutral delay difference equation:

$$
\begin{aligned}
& \Delta^{3}\left(x(n)+\frac{n-2}{2 n} x(n-\tau)+(-1)^{n} \frac{n+1}{n}\right) \\
& +\Delta^{2}\left(\frac{1}{n^{2}+\sqrt{|x(n-1)|}}\right)+\Delta\left(\frac{1}{n^{3}+2 x^{2}\left(n^{2}-n\right)}\right)
\end{aligned}
$$

$$
\begin{gathered}
+\frac{1}{n^{4}+x^{4}(n-2)} \\
=\frac{(-1)^{n}}{n^{6}+n^{4}-1}, \quad n \geq 3,
\end{gathered}
$$

where $\tau \in \mathbb{N} \backslash\{3\}$ is fixed. Let $n_{0}=3, k=1, \beta=\min \{\mid 3-$ $\tau \mid, 1\}=1, A=3, B=12, b^{*}=1 / 2, c^{*}=3, B^{*}=14, A_{*}=1$, and

$$
\begin{array}{r}
b(n)=\frac{n-2}{2 n}, \quad c(n)=(-1)^{n} \frac{n+1}{n}, \\
f(n, u)=\frac{1}{n^{4}+u^{4}}, \quad g(n, u)=\frac{1}{n^{3}+2 u^{2}}, \\
h(n, u)=\frac{1}{n^{2}+\sqrt{|u|}}, \quad d(n)=\frac{(-1)^{n}}{n^{6}+n^{4}+1}, \\
h_{1}(n)=n-1, \quad g_{1}(n)=n^{2}-n, \\
f_{1}(n)=n-2, \quad F_{n}=\frac{1}{n^{4}}, \\
G_{n}=\frac{1}{n^{3}}, \quad H_{n}=\frac{1}{n^{2}}, \\
\forall(n, u) \in \mathbb{N}_{n_{0}} \times \mathbb{R} .
\end{array}
$$

Note that for any $p>2$ and $q>3$

$$
\begin{aligned}
0 & \leq \frac{1}{n} \max \left\{\sum_{i=n}^{\infty} \sum_{t=i}^{\infty} \frac{1}{t^{p}}, \sum_{i=n}^{\infty} \sum_{s=i}^{\infty} \sum_{t=s}^{\infty} \frac{1}{t^{q}}\right\} \\
& \leq \frac{1}{n} \max \left\{\sum_{t=n}^{\infty} \frac{1}{t^{p-1}}, \sum_{t=n}^{\infty} \frac{1}{t^{q-2}}\right\} \longrightarrow 0 \text { as } n \longrightarrow \infty .
\end{aligned}
$$

It is easy to see that (14)-(17) are satisfied. It follows from Theorem 5 that (120) possesses a positive solution $x=$ $\{x(n)\}_{n \in \mathbb{N}_{\beta}} \in l_{\beta}^{\infty}$ satisfying (18) and (19). Moreover, (120) possesses uncountably many positive solutions in $l_{\beta}^{\infty}$.

Example 2. Consider the third order nonlinear neutral delay difference equation:

$$
\begin{aligned}
& \Delta^{3}\left(x(n)+\left(5+\frac{1}{2 n}\right) x(n-\tau)+2+\frac{1}{2 n}\right) \\
& +\Delta^{2}\left(\frac{1}{n^{3}+(n+1) x^{6}(2 n-3)}\right) \\
& +\Delta\left(\frac{2}{2 n^{4}+|x(n+5)|+2}\right)
\end{aligned}
$$




$$
\begin{gathered}
+\frac{\sin \left[n^{3} x\left(n^{2}-n\right)\right]}{n^{6}+x^{2}\left(n^{2}-n\right)} \\
=\frac{n^{2}-1}{n^{6}+n^{3}+2}, \quad n \geq 2,
\end{gathered}
$$

where $\tau \in \mathbb{N} \backslash\{2\}$ is fixed. Let $n_{0}=2, k=1, \beta=\min \{\mid 2-$ $\tau \mid, 1\}=1, A=5, B=200, b^{*}=6, b_{*}=5, c^{*}=4, B^{*}=204$, $A_{*}=1$, and

$$
\begin{array}{r}
b(n)=5+\frac{1}{2 n}, \quad c(n)=2+\frac{1}{2 n}, \\
f(n, u)=\frac{\sin \left(n^{3} u\right)}{n^{6}+u^{2}}, \quad g(n, u)=\frac{2}{2 n^{4}+|u|+2}, \\
h(n, u)=\frac{1}{n^{3}+(n+1) u^{6}}, \quad d(n)=\frac{n^{2}-1}{n^{6}+n^{3}+2}, \\
f_{1}(n)=n^{2}-n, \quad g_{1}(n)=n+5, \\
h_{1}(n)=2 n-3, \quad F_{n}=\frac{1}{n^{6}}, \\
G_{n}=\frac{1}{n^{4}}, \quad H_{n}=\frac{1}{n^{3}}, \\
(n, u) \in \mathbb{N}_{n_{0}} \times \mathbb{R} .
\end{array}
$$

It follows from (122) that (15)-(17) and (40) hold. Thus Theorem 6 ensures that (123) possesses a positive solution $x=\{x(n)\}_{n \in \mathbb{N}_{\beta}} \in l_{\beta}^{\infty}$ satisfying (19) and (41). Moreover, (123) possesses uncountably many positive solutions in $l_{\beta}^{\infty}$.

Example 3. Consider the third order nonlinear neutral delay difference equation:

$$
\begin{aligned}
& \Delta^{3}\left(x(n)+\frac{2-n}{2 n} x(n-\tau)+\frac{64}{64-15 n}\right) \\
& +\Delta^{2}\left(\frac{4 \cos x\left(n^{2}-2\right)}{2 n^{3}+|x(2 n-1)|}\right) \\
& \cdot \Delta\left(\frac{1}{n^{6}+\sqrt{\left|x\left(n^{2}+n\right)\right|} x^{4}\left(n^{2}-2 n\right)}\right) \\
& \left.+\frac{\sin x^{2}(2 n-7)}{n^{4}+x^{4}(3 n-8)}\right) \\
& =\frac{\sqrt{n+1}-\ln n}{n^{8}+n^{5}+3}, \quad n \geq 4,
\end{aligned}
$$

where $\tau \in \mathbb{N} \backslash\{4\}$ is fixed. Let $n_{0}=4, k=2, \beta=\min \{\mid 4-$ $\tau \mid, 1\}=1, A=30, B=300, b^{*}=-1 / 4, b_{*}=-1 / 2, c^{*}=20$, $A_{*}=10, B^{*}=320$, and

$$
b(n)=\frac{2-n}{2 n}, \quad c(n)=\frac{64}{64-15 n}
$$$$
f(n, u, v)=\frac{\sin u^{2}}{n^{4}+v^{4}}, \quad g(n, u, v)=\frac{1}{n^{6}+\sqrt{|u|} v^{4}}
$$$$
h(n, u, v)=\frac{4 \cos v}{2 n^{3}+|u|}, \quad d(n)=\frac{\sqrt{n+1}-\ln n}{n^{8}+n^{5}+3}
$$

$$
f_{1}(n)=2 n-7, \quad f_{2}(n)=3 n-8,
$$$$
g_{1}(n)=n^{2}+n, \quad g_{2}(n)=n^{2}-2 n,
$$$$
h_{1}(n)=2 n-1, \quad h_{2}(n)=n^{2}-2,
$$$$
F_{n}=\frac{1}{n^{4}}, \quad G_{n}=\frac{1}{n^{6}}, \quad H_{n}=\frac{2}{n^{3}},
$$$$
(n, u, v) \in \mathbb{N}_{n_{0}} \times \mathbb{R}^{2} .
$$

It follows from (122) that (15)-(17) and (63) hold. Thus Theorem 7 ensures that (125) possesses a positive solution $x=\{x(n)\}_{n \in \mathbb{N}_{\beta}} \in l_{\beta}^{\infty}$ satisfying (19) and (64). Moreover, (125) possesses uncountably many positive solutions in $l_{\beta}^{\infty}$.

Example 4. Consider the third order nonlinear neutral delay difference equation

$$
\begin{aligned}
& \Delta^{3}\left(x(n)+\frac{1-10 n^{2}-10 n}{n^{2}+n} x(n-\tau)+\frac{2 n+2}{n^{2}}\right) \\
& +\Delta^{2}\left(\frac{\sin x\left(2 n^{2}-1\right)}{n^{4}+2\left|x\left(n^{2}+2 n\right)\right|}\right) \\
& +\Delta\left(\frac{1}{n^{7}+|x(n+10)|^{3}+x^{2}(5 n-4)}\right) \\
& +\frac{\cos 2 x\left(n^{2}+3\right)}{n^{5}+x^{2}\left(4 n^{2}-1\right)} \\
& =\frac{1}{n^{6}+2 n^{3}+8}, \quad n \geq 1,
\end{aligned}
$$


where $\tau \in \mathbb{N} \backslash\{1\}=1$ is fixed. Let $n_{0}=1, k=2, \beta=$ $\min \{|1-\tau|, 1\}, A=10, B=200, b^{*}=-4, b_{*}=-5, c^{*}=5$, $A_{*}=5, B^{*}=205$, and

$$
\begin{gathered}
b(n)=\frac{1-10 n^{2}-10 n}{n^{2}+n}, \quad c(n)=\frac{2 n+2}{n^{2}}, \\
f(n, u, v)=\frac{\cos 2 u}{n^{5}+v^{2}}, \quad g(n, u, v)=\frac{3}{n^{7}+|u|^{3}+v^{2}}, \\
h(n, u, v)=\frac{\sin v}{n^{4}+2|u|}, \quad d(n)=\frac{1}{n^{6}+2 n^{3}+8}, \\
f_{1}(n)=n^{2}+3, \quad f_{2}(n)=4 n^{2}, \\
g_{1}(n)=n+10, \quad g_{2}(n)=5 n-4, \\
h_{1}(n)=n^{2}+2 n, \quad h_{2}(n)=2 n^{2}-1, \\
F_{n}=\frac{1}{n^{5}}, \quad G_{n}=\frac{1}{n^{7}}, \quad H_{n}=\frac{1}{n^{4}}, \\
(n, u, v) \in \mathbb{N}_{n_{0}} \times \mathbb{R}^{2} .
\end{gathered}
$$

It follows from (122) that (15)-(17) and (68) hold. Thus Theorem 8 ensures that (127) possesses a positive solution $x=\{x(n)\}_{n \in \mathbb{N}_{\beta}} \in l_{\beta}^{\infty}$ satisfying (19) and (69). Moreover, (127) possesses uncountably many positive solutions in $l_{\beta}^{\infty}$.

Example 5. Consider the third order nonlinear neutral delay difference equation:

$$
\begin{aligned}
& \Delta^{3}\left(x(n)-x(n-\tau)+\frac{n+1}{n^{3}}\right) \\
& +\Delta^{2}\left(\frac{1}{n^{3}+x^{2}(5 n-4)}\right)+\Delta\left(\frac{1}{n^{6}+2 x^{8}\left(n^{2}-n+1\right)}\right) \\
& +\frac{\sin x^{3}(3 n-2)}{n^{8}+3}=\frac{(-1)^{n(n+1) / 2}}{n^{10}+n^{2}+3}, \quad n \geq 1,
\end{aligned}
$$

where $\tau \in \mathbb{N} \backslash\{1\}$ is fixed. Let $n_{0}=1, k=1, \beta=1, A=3$, $B=5, c^{*}=2, A_{*}=1, B^{*}=7$, and

$$
\begin{array}{r}
b(n)=-1, \quad c(n)=\frac{n+1}{n^{3}}, \\
f(n, u)=\frac{\sin u^{3}}{n^{8}+3}, \quad g(n, u)=\frac{1}{n^{6}+2 u^{8}}, \\
h(n, u)=\frac{1}{n^{3}+u^{2}}, \quad d(n)=\frac{(-1)^{n(n+1) / 2}}{n^{10}+n^{2}+3}, \\
f_{1}(n)=3 n-2, \quad g_{1}(n)=n^{2}-n+1,
\end{array}
$$

$$
\begin{array}{r}
h_{1}(n)=5 n-4, \quad F_{n}=\frac{1}{n^{8}}, \\
G_{n}=\frac{1}{n^{6}}, \quad H_{n}=\frac{1}{n^{3}}, \\
\forall(n, u) \in \mathbb{N}_{n_{0}} \times \mathbb{R}^{+} \backslash\{0\} .
\end{array}
$$

It follows from (122) that (15) and (78)-(81) are satisfied. Thus Theorem 9 ensures that (129) possesses a positive solution $x=\{x(n)\}_{n \in \mathbb{N}_{\beta}} \in l_{\beta}^{\infty}$ satisfying (19) and (82). Moreover, (129) possesses uncountably many positive solutions in $l_{\beta}^{\infty}$.

Example 6. Consider the third order nonlinear neutral delay difference equation:

$$
\begin{aligned}
& \Delta^{3}\left(x(n)+x(n-\tau)+\frac{2 n+4}{n^{3}}\right) \\
& +\Delta^{2}\left(\frac{1}{n^{4}+2 x^{2}(3 n-4)}\right)+\Delta\left(\frac{1}{n^{8}+\left|x^{3}(n-2)\right|}\right) \\
& +\frac{\sin \left[5 x\left(n^{2}-3\right)\right]}{n^{5}+8}=\frac{1}{n^{8}+n^{5}+5}, \quad n \geq 2,
\end{aligned}
$$

where $\tau \in \mathbb{N} \backslash\{2\}$ is fixed. Let $n_{0}=2, k=1, \beta=1, A=100$, $B=101, c^{*}=1, A_{*}=99, B^{*}=102$, and

$$
\begin{array}{r}
b(n)=1, \quad c(n)=\frac{2 n+4}{n^{3}}, \\
f(n, u)=\frac{\sin (5 u)}{n^{5}+8}, \quad g(n, u)=\frac{1}{n^{8}+|u|^{3}}, \\
h(n, u)=\frac{1}{n^{4}+2 u^{2}}, \quad d(n)=\frac{1}{n^{8}+n^{5}+5}, \\
f_{1}(n)=n^{2}-3, \quad g_{1}(n)=n-2, \\
h_{1}(n)=3 n-4, \quad F_{n}=\frac{1}{n^{5}}, \\
G_{n}=\frac{1}{n^{8}}, \quad H_{n}=\frac{1}{n^{4}}, \\
(n, u) \in \mathbb{N}_{n_{0}} \times \mathbb{R} .
\end{array}
$$

It follows from (122) that (15)-(17), (80), and (100) hold. Thus Theorem 10 ensures that (131) possesses a positive solution $x=\{x(n)\}_{n \in \mathbb{N}_{\beta}} \in l_{\beta}^{\infty}$ satisfying (19) and (103). Moreover, (131) possesses uncountably many positive solutions in $l_{\beta}^{\infty}$.

\section{Conflict of Interests}

The authors declare that there is no conflict of interests regarding the publication of this paper. 


\section{Acknowledgments}

The authors would like to thank the referees for useful comments and suggestions. This study was supported by research funds from Dong-A University.

\section{References}

[1] A. Andruch-Sobilo and M. Migda, "On the oscillation of solutions of third order linear difference equations of neutral type," Mathematica Bohemica, vol. 130, no. 1, pp. 19-33, 2005.

[2] S. R. Grace and G. G. Hamedani, "On the oscillation of certain neutral difference equations," Mathematica Bohemica, vol. 125, no. 3, pp. 307-321, 2000.

[3] Z. Liu, H. Wu, S. M. Kang, and Y. C. Kwun, "On positive solutions and Mann iterative schemes of a third order difference equation," Abstract and Applied Analysis, vol. 2014, Article ID 470181, 16 pages, 2014.

[4] S. H. Saker, "Oscillation of third-order difference equations," Portugaliae Mathematica, vol. 61, no. 3, pp. 249-257, 2004.

[5] J. Yan and B. Liu, "Asymptotic behavior of a nonlinear delay difference equation," Applied Mathematics Letters, vol. 8, no. 6, pp. 1-5, 1995.

[6] Z.-Q. Zhu, G.-Q. Wang, and S. S. Cheng, "A classification scheme for nonoscillatory solutions of a higher order neutral difference equation," Advances in Difference Equations, vol. 2006, Article ID 047654, 19 pages, 2006. 


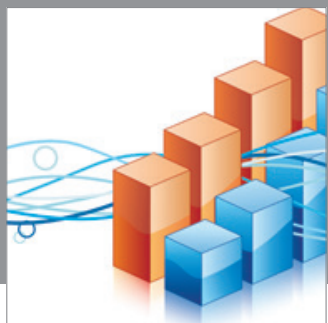

Advances in

Operations Research

mansans

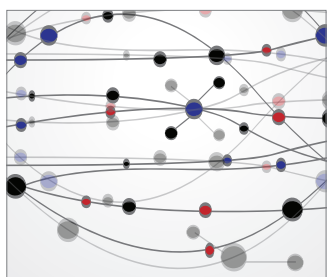

The Scientific World Journal
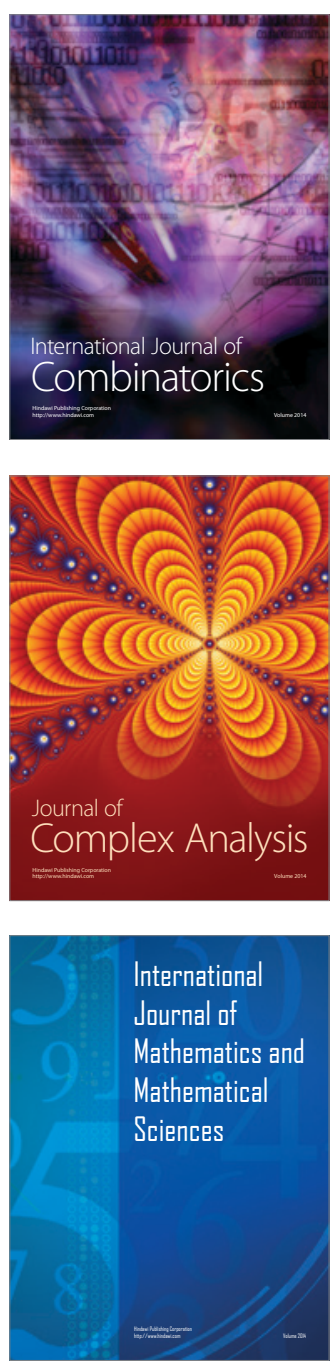
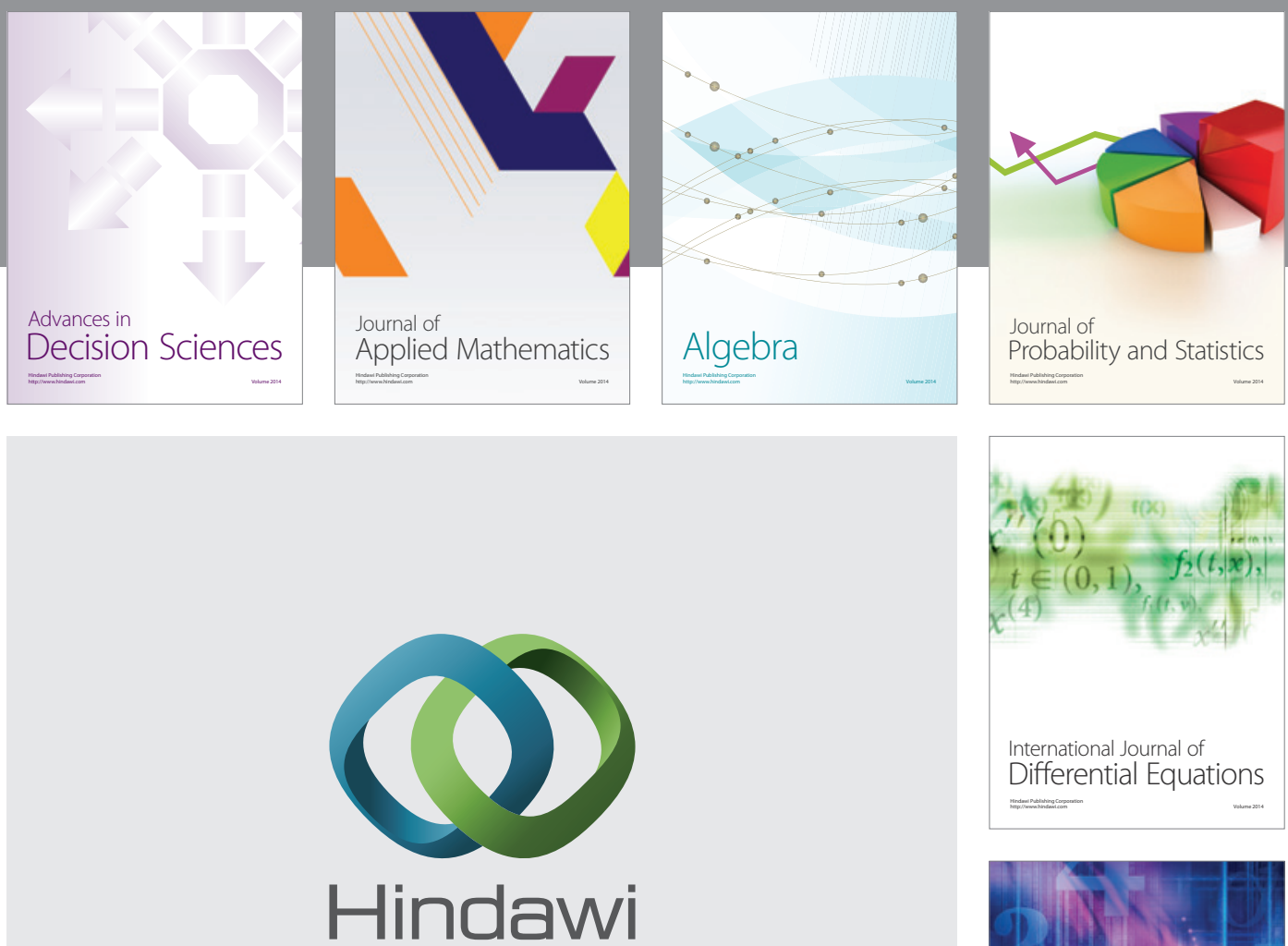

Submit your manuscripts at http://www.hindawi.com
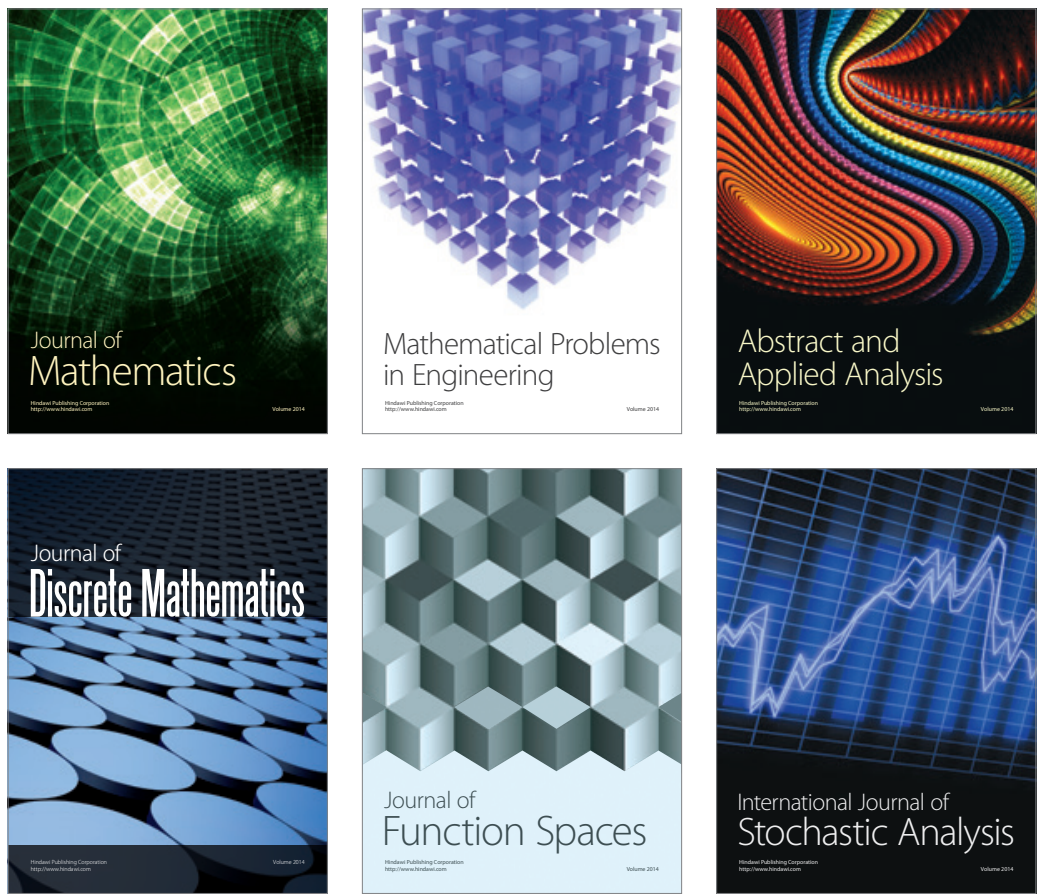

Journal of

Function Spaces

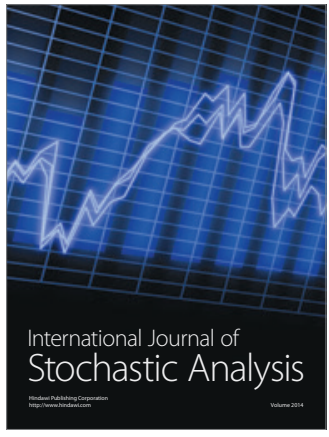

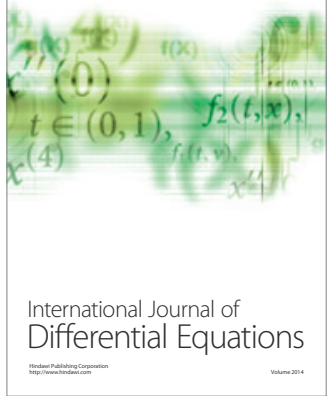
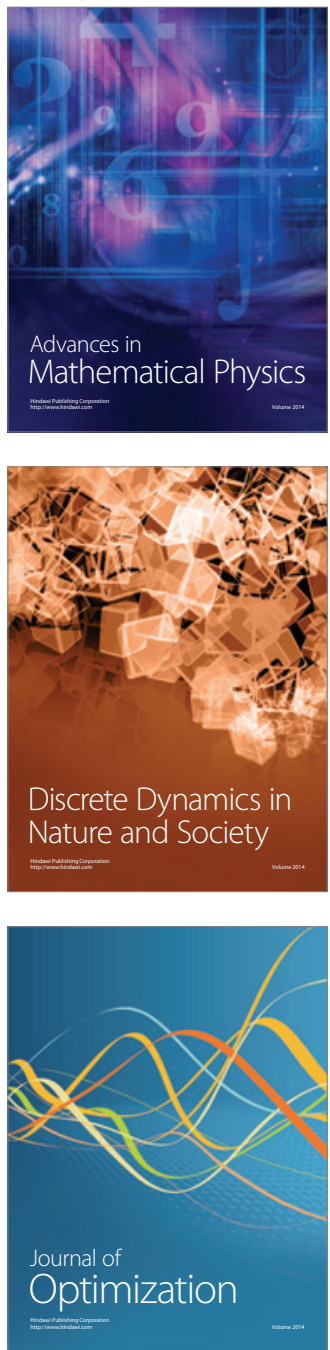